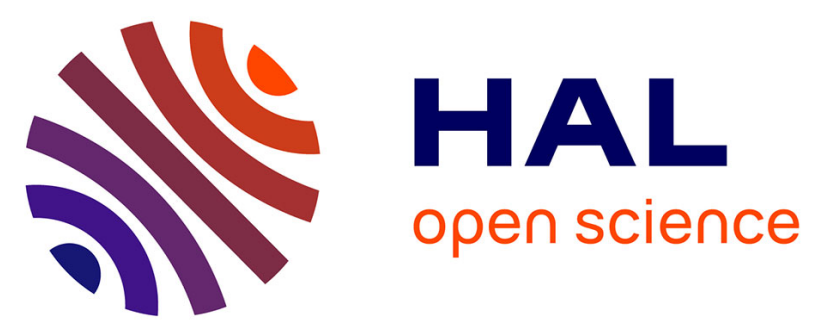

\title{
Étude théorique de la compression de spin nucléaire par mesure quantique non destructive en continu
}

Alan Serafin, Yvan Castin, Matteo Fadel, Philipp Treutlein, Alice Sinatra

\section{To cite this version:}

Alan Serafin, Yvan Castin, Matteo Fadel, Philipp Treutlein, Alice Sinatra. Étude théorique de la compression de spin nucléaire par mesure quantique non destructive en continu. Comptes Rendus. Physique, 2021, 22, pp.1-35. 10.5802/crphys.71 . hal-03083577v2

\section{HAL Id: hal-03083577 \\ https://hal.science/hal-03083577v2}

Submitted on 15 Apr 2021

HAL is a multi-disciplinary open access archive for the deposit and dissemination of scientific research documents, whether they are published or not. The documents may come from teaching and research institutions in France or abroad, or from public or private research centers.
L'archive ouverte pluridisciplinaire HAL, est destinée au dépôt et à la diffusion de documents scientifiques de niveau recherche, publiés ou non, émanant des établissements d'enseignement et de recherche français ou étrangers, des laboratoires publics ou privés.

\section{(c)(1)}

Distributed under a Creative Commons Attribution| 4.0 International License 


\title{
Étude théorique de la compression de spin nucléaire par mesure quantique non destructive en continu - Nuclear spin squeezing by continuous quantum non-demolition measurement : a theoretical study
}

\author{
Alan Serafin ${ }^{\mathrm{a}}$, Yvan Castin ${ }^{\mathrm{a}}$, Matteo Fadel ${ }^{\mathrm{b}}$, Philipp Treutlein ${ }^{\mathrm{b}}$, Alice Sinatra ${ }^{\mathrm{a}}$ \\ ${ }^{a}$ Laboratoire Kastler Brossel, ENS-Université PSL, CNRS, Université de la Sorbonne et Collège de France, 24 rue Lhomond, 75231 Paris, France \\ ${ }^{b}$ Département de physique, Université de Bâle, Klingelbergstrasse 82, 4056 Bâle, Suisse
}

\section{Résumé}

Nous proposons de tirer parti du très faible couplage du spin nucléaire de l'hélium 3 fondamental à son environnement pour produire des états quantiques macroscopiques à très longue durée de vie, ici des états comprimés du spin nucléaire, dans un gaz en cellule à température ordinaire. Pour effectuer une mesure quantique non destructive sur une composante transverse du spin nucléaire collectif préalablement polarisé, on allume temporairement une décharge dans le gaz, ce qui peuple l'état métastable de l'hélium 3. Le spin collectif correspondant au niveau $F=1 / 2$ des métastables s'hybride alors légèrement avec celui des fondamentaux par collision d'échange de métastabilité. Il reste à mesurer en continu le champ lumineux sortant d'une cavité optique, où il a interagi de manière dispersive avec le spin collectif du métastable, pour accéder aux fluctuations du spin nucléaire. Dans un modèle de trois spins collectifs couplés (nucléaire, du métastable et de Stokes de la lumière) dans l'approximation de Primakoff, et pour deux schémas de mesure, nous calculons les moments de la composante comprimée $I_{z}$ du spin nucléaire collectif conditionnés au signal optique moyenné sur le temps d'observation $t$. Dans le schéma de comptage de photons, nous retrouvons que l'observable comprimée est $I_{z}^{2}$ plutôt que $I_{z}$. Dans le schéma de détection homodyne, nous résolvons analytiquement l'équation stochastique sur l'état du système conditionné à la mesure; la moyenne conditionnelle de $I_{z}$ dépend linéairement du signal et la variance conditionnelle de $I_{z}$ n'en dépend pas. La variance conditionnelle décroît comme $\left(\Gamma_{\mathrm{sq}} t\right)^{-1}$, où le taux de compression $\Gamma_{\mathrm{sq}}$, que nous calculons explicitement, est linéaire en l'intensité lumineuse dans la cavité à faible couplage atome-champ et sature à fort couplage au taux effectif d'échange de métastabilité dans l'état fondamental, proportionnel à la densité d'atomes métastables. Enfin, nous tenons compte de la désexcitation des métastables sur les parois, qui induit une décohérence du spin nucléaire avec un taux ramené $\gamma_{\alpha}$. Elle impose une limite $\propto\left(\gamma_{\alpha} / \Gamma_{\mathrm{sq}}\right)^{1 / 2}$ sur la variance conditionnelle atteinte en un temps $\propto\left(\gamma_{\alpha} \Gamma_{\mathrm{sq}}\right)^{-1 / 2}$. Une version multilingue est disponible sur l'archive ouverte HAL à l'adresse https://hal . archives-ouvertes .fr/hal-03083577.

Mots-clés : compression de spin; hélium 3; spin nucléaire; métrologie quantique; fonctions d'onde stochastiques

\begin{abstract}
We propose to take advantage of the very weak coupling of the ground-state helium-3 nuclear spin to its environment to produce very long-lived macroscopic quantum states, here nuclear spin squeezed states, in a gas cell at room temperature. To perform a quantum non-demolition measurement of a transverse component of the previously polarized collective nuclear spin, a discharge is temporarily switched on in the gas, which populates helium-3 metastable state. The collective spin corresponding to the $F=1 / 2$ metastable level then hybridizes slightly with the one in the ground state by metastability exchange collisions. To access the nuclear spin fluctuations, one continuously measures the light field leaking out of an optical cavity, where it has interacted dispersively with the metastable state collective spin. In a model of three coupled collective spins (nuclear, metastable and Stokes for light) in the Primakoff approximation, and for two measurement schemes, we calculate the moments of the collective nuclear spin squeezed component $I_{z}$ conditioned on the optical signal averaged over the observation time $t$. In the photon counting scheme, we find that the squeezed observable is $I_{z}^{2}$ rather than $I_{z}$. In the homodyne detection scheme, we analytically solve the stochastic equation for the state of the system conditioned to the measurement; the conditional expectation value of $I_{z}$ depends linearly on the signal and the conditional variance of $I_{z}$ does not depend on it. The conditional variance decreases as $\left(\Gamma_{\mathrm{sq}} t\right)^{-1}$, where the squeezing rate $\Gamma_{\mathrm{sq}}$, which we calculate explicitly, depends linearly on the light intensity in the cav-
\end{abstract}


ity at weak atom-field coupling and saturates at strong coupling to the ground state metastability exchange effective rate, proportional to the metastable atom density. Finally, we take into account the de-excitation of metastable atoms at the walls, which induces nuclear spin decoherence with an effective rate $\gamma_{\alpha}$. It imposes a limit $\propto\left(\gamma_{\alpha} / \Gamma_{\mathrm{sq}}\right)^{1 / 2}$ on the conditional variance reached in a time $\propto\left(\gamma_{\alpha} \Gamma_{\mathrm{sq}}\right)^{-1 / 2}$. A multilingual version is available on the open archive HAL at https: //hal.archives-ouvertes.fr/hal-03083577.

Keywords: spin squeezing ; helium 3 ; nuclear spin ; quantum metrology ; stochastic wave functions

\section{Introduction}

L'hélium 3 dans son état fondamental jouit de la propriété remarquable d'avoir un spin 1/2 purement nucléaire donc parfaitement isolé du monde extérieur, même dans un environnement aussi hostile aux cohérences quantiques que celui d'un gaz d'hélium dans une cellule centimétrique à température ordinaire et à une pression de l'ordre du millibar. Par des techniques de polarisation nucléaire bien maîtrisées, atteignant un taux de $90 \%$, on peut préparer alors de manière routinière (par exemple à des fins d'imagerie pulmonaire par résonance magnétique nucléaire [1]) un spin nucléaire collectif géant avec une durée de vie extrêmement longue. Ainsi, un temps de cohérence $T_{2}$ supérieur à 60 heures a été mesuré dans des dispositifs de magnétométrie ultra-précise [2], et semble limité seulement par le temps de décroissance longitudinal $T_{1}$ dû aux collisions avec les parois. ${ }^{1}$ Ces valeurs font du spin nucléaire macroscopique dans un gaz à l'ambiante un système idéal pour la production, l'étude et l'utilisation d'états intriqués, et donc un compétiteur des gaz d'atomes froids et des condensats de Bose-Einstein en métrologie et traitement quantique de l'information [4]. Déjà en 2005, nous avions pressenti que les spins nucléaires de l'hélium 3 pourraient donner naissance à des mémoires quantiques [5] ou à des états quantiques non locaux [6] de très longue durée de vie. Depuis, des percées expérimentales ont été accomplies dans le domaine de la compression de spin, notamment au moyen de mesures quantiques non destructives (QND) dans des gaz atomiques d'alcalins interagissant avec un mode du champ électromagnétique [4, $7,8,9]$, qui ont permis d'obtenir récemment un état comprimé de spin d'une durée de vie d'une seconde dans l'état hyperfin fondamental du rubidium dans des conditions métrologiques [10]. On rappelle que, par analogie avec les états comprimés de la lumière en optique quantique, un spin (ici collectif) $\vec{I}$ est dit être dans un état comprimé si, dans une direction $O z$ orthogonale à celle $O x$ du spin moyen, il admet des fluctuations réduites par rapport à la limite quantique standard ${ }^{2}$; ceci entraîne une précision accrue dans le pointage angulaire du spin moyen dans cette direction, donc de sa fréquence de précession autour de l'axe $O y$, dans tout dispositif métrologique de type horloge atomique ou magnétomètre $[11,12]$ qui s'est suffisamment affranchi des bruits techniques pour atteindre la limite quantique standard [13].

Transposer la technique de compression par mesure QND des spins hyperfins des alcalins au spin nucléaire de l'hélium 3 représente cependant un réel défi, en raison même de la particularité dudit spin, son faible couplage à l'environnement. L'état fondamental singulet de l'hélium 3, séparé en énergie d'environ 20 eV du premier état excité, n'est pas directement accessible par laser. Cependant, au moyen d'une décharge oscillante, une petite fraction des atomes du gaz, de l'ordre de $10^{-6}$, peut être portée dans l'état triplet métastable, un excellent point de départ pour des transitions optiques dans le proche infrarouge. L'orientation des spins nucléaires s'obtient alors au travers d'un processus indirect, le pompage optique par échange de métastabilité [3]. Dans un premier temps, le moment cinétique est transféré par interaction laser-matière des photons aux atomes métastables, a priori à leur spin électronique (le seul à être fortement couplé au champ laser) mais a posteriori aussi à leur spin nucléaire grâce au couplage hyperfin. Dans un deuxième temps, on tire parti des collisions d'échange de métastabilité entre atomes métastables et atomes dans l'état fondamental pour orienter les spins nucléaires dans l'état fondamental, avec une échelle de temps limitée par la faible densité des atomes dans l'état métastable et de l'ordre de la seconde. Même si la collision d'échange de métastabilité peut transférer des corrélations quantiques (voir les références $[5,6]$ ), on ne peut s'attendre à ce

1. Des temps $T_{1}$ de plusieurs centaines d'heures peuvent même être obtenus [3].

2. Le bruit quantique standard correspond à des écarts-types $\Delta I_{y}^{\text {st }}=\Delta I_{z}^{\text {st }}=\left(\left|\left\langle I_{x}\right\rangle\right| / 2\right)^{1 / 2}$, c'est-à-dire au cas d'égalité avec symétrie de révolution des fluctuations autour du spin moyen dans l'inégalité de Heisenberg $\Delta I_{y} \Delta I_{z} \geq\left|\left\langle I_{x}\right\rangle\right| / 2$, nos spins étant ici sans dimension. Une discussion plus approfondie montre que, dans la géométrie considérée, le rapport bruit sur signal dans une mesure de fréquence de précession est en fait proportionnel à $\xi=(2 I)^{1 / 2} \Delta I_{z} /\left|\left\langle I_{x}\right\rangle\right|$ (ceci fait apparaître naturellement l'angle des fluctuations de la direction du spin dans le plan $x O z$ ) plutôt qu'au rapport $\Delta I_{z} / \Delta I_{z}^{\text {st }}$ naïvement attendu [11]. Dans le schéma que nous proposons, nous dégradons $\xi^{2}$ d'un facteur $\approx 2$ en utilisant un état de spin nucléaire partiellement polarisé $\left\langle I_{x}\right\rangle / I<1$, voir la figure 3, mais nous ambitionnons de gagner beaucoup plus avec la compression de spin. 
(a) cellule de gaz d'hélium 3 dans une cavité optique

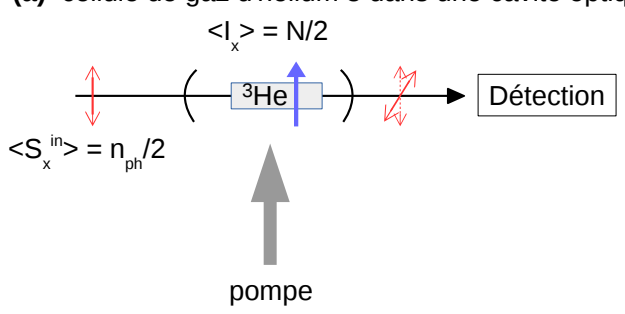

(b) comptage de photons

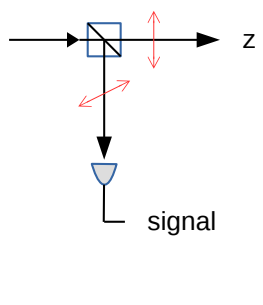

(c) détection homodyne

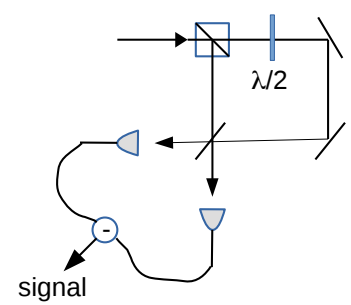

Figure 1 - Vue d'ensemble du dispositif. (a) Cellule en verre centimétrique remplie d'un gaz d'hélium 3 à température ambiante et placée dans une cavité optique d'axe $O z$ (axe horizontal sur la figure). Le spin de Stokes de la lumière et les spins atomiques (nucléaires et des métastables) sont polarisés linéairement selon $O x$ (axe vertical sur la figure). Le mode du champ en cavité polarisé selon $O y$, initialement vide, se peuple par effet Faraday sous l'action des fluctuations quantiques du spin des métastables selon $O z$ lors de sa propagation dans le gaz. On le mesure en continu à l'extérieur de la cavité par l'une des deux méthodes suivantes : (b) les photons sortant de la cavité polarisés selon $O y$ sont séparés de ceux polarisés selon $O x$ par un cube polariseur puis détectés dans le régime de comptage de photons; (c) on effectue une détection homodyne d'une quadrature du champ sortant polarisé selon $O y$, en utilisant comme oscillateur local le champ sortant polarisé selon $O x$ (dont on a fait tourner au préalable la polarisation avec une lame demi-onde pour l'amener selon $O y$ ).

qu'une seule mesure sur une petite fraction des atomes $\left(10^{-6}\right)$ projette l'ensemble du système dans un état comprimé. La solution que nous proposons est d'effectuer une mesure QND en continu démultipliée par une cavité optique résonnante dans laquelle est placée la cellule. En effet, bien que les atomes métastables aient individuellement une durée de vie relativement courte (ils perdent leurs corrélations quantiques et retombent dans l'état fondamental à chaque collision avec les parois de la cellule), faire une mesure destructive en continu de la lumière qui sort de la cavité après interaction avec les atomes métastables revient à effectuer une mesure QND en continu sur le spin nucléaire collectif dans l'état fondamental, ce qui le prépare dans l'état comprimé souhaité sans affecter sa durée de vie. ${ }^{3}$ Comme nous le verrons, notre procédure de réduction des fluctuations de spin se résume plus précisément par le diagramme :

\begin{tabular}{|c|c|c|c|c|}
\hline $\begin{array}{l}\text { spin collectif } \vec{I} \text { dans } \\
\text { l'état fondamental }\end{array}$ & $\underset{\text { de métastabilité }}{\stackrel{\text { collisions d'echange }}{\longleftrightarrow}}$ & \begin{tabular}{|l|}
$\begin{array}{l}\text { spin collectif dans l'état } \\
\text { métastable }\end{array}$ \\
\end{tabular} & $\stackrel{\text { effet Faraday }}{\longleftrightarrow}$ & $\begin{array}{l}\text { champ en cavité po- } \\
\text { larisé selon } O x\end{array}$ \\
\hline $\begin{array}{l}\text { réduction des fluctua- } \\
\text { tions de } I_{z}^{2}, I_{z}\end{array}$ & & $\begin{array}{l}\text { mesure en continu non } \\
\text { destructive de } I_{z}^{2}, I_{z}\end{array}$ & $\begin{array}{l}\text { comptage de photons, } \\
\text { détection homodyne }\end{array}$ & $\begin{array}{l}\text { mesure en continu } \\
\text { du champ sortant } \\
\text { polarisé selon } O y\end{array}$ \\
\hline
\end{tabular}

Ce travail donne une présentation théorique détaillée du mécanisme de compression et de ses limites; une étude de faisabilité plus pointue prenant en compte les valeurs expérimentalement accessibles des paramètres est effectuée dans la référence [14]. Très récemment, des idées similaires ont été mises en avant dans un système physique différent, le mélange alcalin-gaz rare $[15,16]$. Nous sommes confiants que la manipulation quantique des spins nucléaires à longue durée de vie est promise à un développement rapide, ouvrant de nouvelles perspectives pour la recherche fondamentale et les applications, en particulier en magnétométrie [17, 18, 19, 20,9].

\section{Vue d'ensemble et description semi-classique}

Le système physique considéré est représenté sur la figure 1. Une cellule remplie d'un gaz partiellement polarisé de quelques mbars d'atomes d'hélium 3 pur est placée à l'intérieur d'une cavité optique. Alors que la majorité

3. Ce type de mesure diffère donc de la mesure instantanée des traités de mécanique quantique, qui projette l'état du système dans un sousespace propre de l'observable mesurée selon le postulat de von Neumann. 


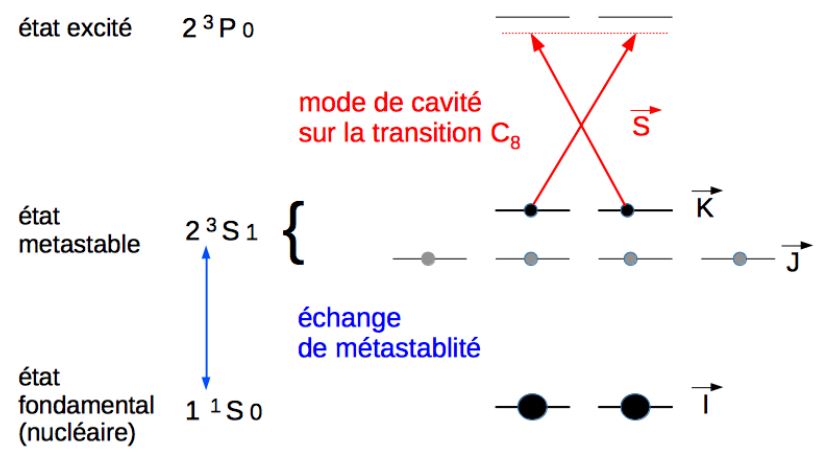

Figure 2 - Niveaux d'énergie utiles de l'atome ${ }^{3} \mathrm{He}$ (les sous-niveaux Zeeman correspondent au choix de $O z$ comme axe de quantification, les atomes étant polarisés selon $O x$ ). Le mode du champ en cavité polarisé selon $O x$ excite la transition $C_{8}$ entre le niveau $F=1 / 2$ de l'état métastable $2^{3} S_{1}$ et le plus haut niveau d'énergie $F=1 / 2$ de l'état excité $2^{3} P$, avec un désaccord en fréquence négatif beaucoup plus grand en valeur absolue que la mi-largeur Doppler de l'état excité (de l'ordre de $1 \mathrm{GHz}$ ), afin que la classe de vitesse résonnante avec le laser soit presque vide, mais beaucoup plus faible que le clivage hyperfin de $6,74 \mathrm{GHz}$ dans l'état métastable (et a fortiori que le clivage fin $2^{3} P_{1}-2^{3} P_{0}$ de $29,6 \mathrm{GHz}$ dans l'état excité), afin que le niveau métastable $F=3 / 2$ soit très peu affecté par le laser. (Note : l'espacement en fréquence ne permet pas de satisfaire largement à ces deux contraintes, et l'on ne peut exclure que le couplage de $F=3 / 2$ au champ n'ait un petit effet sur la dynamique de compression; nous le négligeons ici mais on pourrait en tenir compte avec un hamiltonien plus complet que notre modèle minimal (2), comme celui de la référence [23]. Nous avons par ailleurs vérifié, au moyen de cette référence, qu'il n'existe pas de fréquence laser «magique » loin de résonance permettant d'annuler la contribution du tenseur d'alignement du niveau $F=3 / 2$ à l'opérateur de déplacement lumineux.) Les six sous-niveaux de l'état métastable $2^{3} S_{1}$ sont couplés aux deux sous-niveaux (purement nucléaires) de l'état fondamental $1{ }^{1} S_{0}$ par les collisions d'échange de métastabilité.

des atomes restent dans l'état singulet fondamental $1^{1} S$ de l'hélium, une faible décharge porte une infime fraction des atomes, en général $\simeq 10^{-6}$, dans l'état triplet métastable $2^{3} S$. D'une part, on injecte dans la cavité un faisceau laser se propageant selon l'axe de la cavité $O z$ et polarisé linéairement selon la direction $O x$, qui est également la direction de polarisation de l'échantillon atomique, pour exciter la transition $2^{3} S-2^{3} P$ avec un grand désaccord en fréquence. D'autre part, les atomes dans l'état métastable $2^{3} S$ (de spin hyperfin électronique et nucléaire) sont couplés aux atomes dans l'état fondamental (de spin purement nucléaire) par des collisions d'échange de métastabilité; de façon remarquable, bien que chaque collision d'échange soit individuellement incohérente, ceci conduit à un couplage macroscopique bien défini entre les spins collectifs correspondants [21, 22]. Comme l'interaction de Faraday avec les atomes métastables fait légèrement tourner autour de l'axe $O z$ la polarisation de la lumière initialement dirigée selon $O x$, d'un angle proportionnel à la composante du spin collectif des métastables selon $O z$ comme nous le verrons, une mesure destructive en continu de la composante de polarisation selon $O y$ du champ sortant de la cavité (i) par comptage de photons comme indiqué sur la figure $1 \mathrm{~b}$ ou (ii) par détection homodyne comme sur la figure $1 \mathrm{c}$, réalise in fine une mesure quantique non destructive en continu du spin nucléaire collectif selon $O z$ des atomes d'hélium 3 dans l'état fondamental.

Dans le reste de cette section, par un traitement semi-classique des fluctuations des spins autour de leurs valeurs moyennes dans l'état stationnaire, nous réduisons notre système physique complexe à celui plus simple de trois spins collectifs couplés, dont la section 3 donnera une description quantique.

La structure atomique pertinente de l'atome ${ }^{3} \mathrm{He}$ et les transitions excitées par le champ en cavité sont représentées sur la figure 2. On appelle $\vec{I}$ le spin nucléaire collectif dans l'état fondamental, $\vec{J}$ et $\vec{K}$ les spins collectifs associés aux multiplicités hyperfines $F=3 / 2$ et $F=1 / 2$ dans l'état métastable. Pour la lumière se propageant selon $O z$, nous introduisons le spin de Stokes [23] construit à partir des opérateurs de création et d'annihilation d'un photon dans les 
modes de cavité polarisés linéairement selon $O x$ et $O y:{ }^{4}$

$$
S_{x}=\frac{1}{2}\left(c_{x}^{\dagger} c_{x}-c_{y}^{\dagger} c_{y}\right) \quad ; \quad S_{y}=\frac{1}{2}\left(c_{x}^{\dagger} c_{y}+c_{y}^{\dagger} c_{x}\right) \quad ; \quad S_{z}=\frac{1}{2 \mathrm{i}}\left(c_{x}^{\dagger} c_{y}-c_{y}^{\dagger} c_{x}\right) .
$$

Nous supposons pour simplifier que la cellule est éclairée uniformément par le mode de cavité. Dans la limite d'un grand désaccord et d'une faible saturation de la transition atomique par le champ, l'état excité $2^{3} P$ peut être éliminé adiabatiquement et l'interaction hamiltonienne entre le spin du métastable $\vec{K}$ et le spin de Stokes $\vec{S}$ prend la forme de Faraday [23] :

$$
H=\hbar \chi K_{z} S_{z}
$$

qui n'est autre que l'opérateur de déplacement lumineux des sous-niveaux Zeeman dans le niveau métastable $F=1 / 2$, comme on le voit bien sur la forme de $S_{z}$ dans la note 4. Les équations non linéaires couplées décrivant l'évolution des spins moyens sont données dans l'Annexe A, voir les équations (A.1)-(A.3). Outre l'évolution due à l'hamiltonien de Faraday (2) et aux collisions d'échange de métastabilité, elles incluent la contribution des termes liouvilliens habituels dans l'équation pilote décrivant l'injection d'un champ cohérent polarisé selon $O x$ dans la cavité et les pertes dues au miroir de sortie, dont l'effet combiné conduit à $\left\langle S_{x}\right\rangle=n_{\mathrm{ph}} / 2$ dans l'état stationnaire en l'absence d'atomes, $n_{\mathrm{ph}}$ étant le nombre moyen de photons dans le mode polarisé selon $O x$. Ces équations sont ensuite linéarisées autour d'une solution stationnaire partiellement polarisée (A.8)-(A.9), et les fluctuations du spin $\vec{J}$ et du tenseur d'alignement collectif dans $F=3 / 2$ sont éliminées adiabatiquement ${ }^{5}$ pour obtenir des équations couplées sur les fluctuations des trois spins collectifs $\vec{I}, \vec{K}$ et $\vec{S}$, dont les valeurs moyennes stationnaires sont données par :

$$
\langle\vec{I}\rangle_{s}=\frac{N}{2} \vec{u}_{x} \quad ; \quad\langle\vec{K}\rangle_{s}=\frac{n}{2} \vec{u}_{x} \quad ; \quad\langle\vec{S}\rangle_{s}=\frac{n_{\mathrm{ph}}}{2} \vec{u}_{x}
$$

Ici $\vec{u}_{x}$ est le vecteur unitaire selon $O x, N$ et $n$ sont les nombres effectifs d'atomes fondamentaux et métastables participant à la dynamique des spins collectifs. Comme nous le montrons dans l'Annexe $\mathrm{A}$, ces nombres effectifs sont renormalisés par rapport aux nombres totaux vrais $N_{\text {cell }}$ et $n_{\text {cell }}$ dans la cellule, par des facteurs dépendant de la polarisation :

$$
N=\eta N_{\text {cell }} \quad ; \quad n=\left(\frac{1-\eta^{2}}{3+\eta^{2}}\right) \eta n_{\text {cell }}
$$

où $\eta \in[0,1]$ est la polarisation nucléaire, ${ }^{6}$ et les équations semi-classiques sur les fluctuations des trois spins collectifs s'écrivent :

$$
\begin{aligned}
\frac{\mathrm{d}}{\mathrm{d} t} \delta S_{z} & =-\frac{\kappa}{2} \delta S_{z} & \frac{\mathrm{d}}{\mathrm{d} t} \delta S_{y} & =-\frac{\kappa}{2} \delta S_{y}+\chi\left\langle S_{x}\right\rangle_{s} \delta K_{z} \\
\frac{\mathrm{d}}{\mathrm{d} t} \delta I_{z} & =-\gamma_{f} \delta I_{z}+\gamma_{m} \delta K_{z} & \frac{\mathrm{d}}{\mathrm{d} t} \delta I_{y} & =-\gamma_{f} \delta I_{y}+\gamma_{m} \delta K_{y} \\
\frac{\mathrm{d}}{\mathrm{d} t} \delta K_{z} & =-\gamma_{m} \delta K_{z}+\gamma_{f} \delta I_{z} & \frac{\mathrm{d}}{\mathrm{d} t} \delta K_{y} & =-\gamma_{m} \delta K_{y}+\gamma_{f} \delta I_{y}+\chi\left\langle K_{x}\right\rangle_{s} \delta S_{z}
\end{aligned}
$$

Ici, $\kappa$ est le taux de perte de la cavité, $\gamma_{m}$ et $\gamma_{f}$ sont les taux effectifs d'échange de métastabilité dans l'état métastable et dans l'état fondamental. Ces derniers dépendent de la polarisation nucléaire comme ci-dessous et sur la figure 3a, et sont dans le même rapport que les nombres d'atomes effectifs $N$ et $n$ (4) constituant les spins collectifs :

$$
\gamma_{f}=\frac{4+\eta^{2}}{8-\eta^{2}} \frac{1-\eta^{2}}{3+\eta^{2}} \frac{1}{T} \quad ; \quad \gamma_{m}=\frac{4+\eta^{2}}{8-\eta^{2}} \frac{1}{\tau} \quad ; \quad \frac{\gamma_{m}}{\gamma_{f}}=\frac{N}{n} \gg 1
$$

4. De manière équivalente, on peut construire le spin de Stokes $\vec{S}$ en utilisant les opérateurs d'annihilation dans les modes polarisés circulairement, $c_{1}=\frac{1}{\sqrt{2}}\left(c_{x}-\mathrm{i} c_{y}\right), c_{2}=\frac{1}{\sqrt{2}}\left(c_{x}+\mathrm{i} c_{y}\right)$ [24], auquel cas $S_{z}=\frac{1}{2}\left(c_{1}^{\dagger} c_{1}-c_{2}^{\dagger} c_{2}\right)$.

5. Nous pensons que cette approximation non mathématiquement contrôlée est raisonnable pour l'expérience proposée, car le spin $\vec{J}$ n'est pas directement couplé à la lumière donc n'est pas directement affecté par la mesure du champ en continu. En revanche, si l'on éliminait de même les fluctuations du spin $\vec{K}$, directement couplé au champ, on commettrait une erreur non négligeable sur la dynamique de compression du spin dans le cas de la détection par comptage de photons (revenant à omettre le saut double $C_{d}$ dans l'équation pilote (37) et le taux $\Gamma_{0}$ dans le nombre moyen de photons comptés (45) donc à sous-estimer fortement le nombre de photodétections requises pour atteindre un niveau de compression donné), mais une erreur négligeable dans le cas de la détection homodyne, comme nous l'avons vérifié sur le modèle à un mode de la section 3.4.

6. Notons que $n=0$ dans le cas entièrement polarisé $\eta=1$. En effet, toute la population de l'état métastable se trouve alors dans le sous-niveau Zeeman extrême $m_{x}=3 / 2$ de l'état hyperfin $F=3 / 2$ et la multiplicité $F=1 / 2$ est vide. 

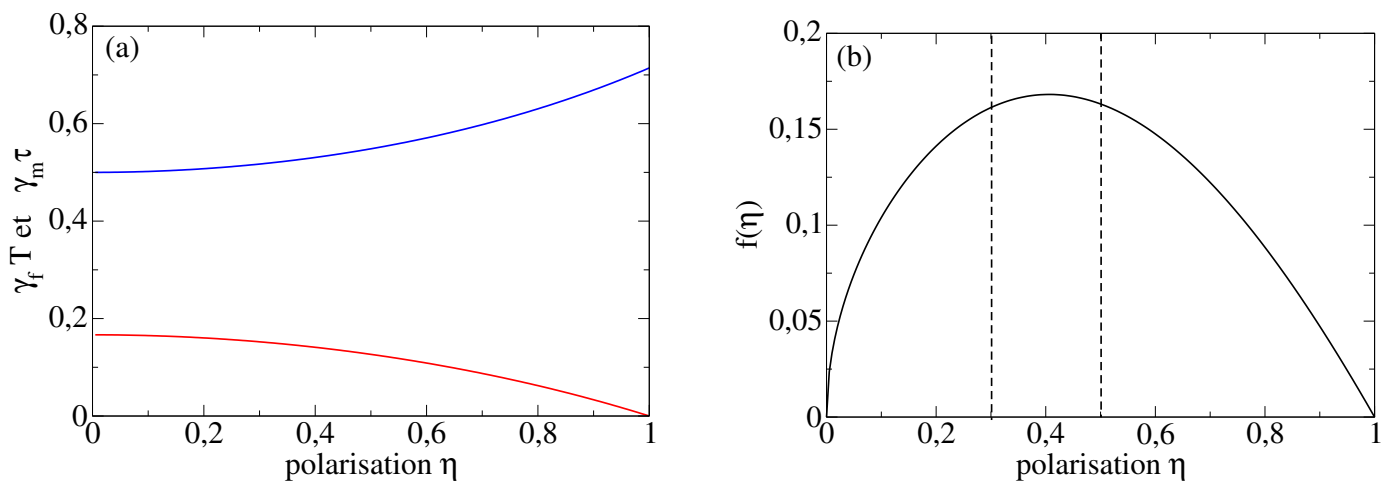

Figure 3 - (a) Taux effectifs d'échange de métastabilité $\gamma_{f}$ (courbe du bas, en rouge) et $\gamma_{m}$ (courbe du haut, en bleu) d'après l'équation (8) dans l'état fondamental et métastable en fonction de la polarisation nucléaire $\eta$, normalisés par les taux des collisions d'échange de métastabilité $1 / T$ et $1 / \tau$ subies par un atome fondamental et métastable dans le gaz. (b) Dépendance en polarisation nucléaire de la pulsation de Faraday $\Omega_{\alpha}$ entrant dans le taux de création d'excitations par le couplage de Faraday dans le mode bosonique nucléaire hybridé (32) et dans les taux de compression de spin (49) et (95), dans la limite $\gamma_{f} \ll \gamma_{m}$; plus précisément, on représente le facteur $f(\eta)=\sqrt{\eta} \frac{1-\eta^{2}}{3+\eta^{2}}$ tel que $\Omega_{\alpha} \simeq \Omega\left(\gamma_{f} / \gamma_{m}\right)^{1 / 2}=\chi \sqrt{n_{\mathrm{ph}} n_{\text {cell }}} \sqrt{\frac{n_{\text {cell }}}{N_{\text {cell }}}} f(\eta)$. Lorsque la polarisation varie entre 0,3 et 0,5 (lignes tiretées verticales), $f(\eta)$ s'écarte de $4 \%$ de son maximum $\simeq 0,17$ atteint en $\eta=0,42$. Il est donc avantageux de se placer près de cette valeur de $\eta$ pour réduire la dérive temporelle de $\Omega_{\alpha}$ due à un léger amortissement de la polarisation nucléaire pendant la compression de spin (en effet, le processus de pompage optique est alors interrompu et la durée de vie de la polarisation est réduite par la présence de la décharge, elle devient de l'ordre de $\gamma_{\alpha}^{-1}$, où $\gamma_{\alpha}$ est le taux de décohérence ramené de la section 4.2.4).

les taux individuels des collisions d'échange de métastabilité $1 / T$ et $1 / \tau$ subies par un atome dans l'état fondamental et dans l'état métastable étant proportionnels à $n_{\text {cell }}$ et $N_{\text {cell }}$ avec la même constante de proportionnalité. Sur la figure 3 b, nous montrons également la dépendance en polarisation nucléaire du couplage de Faraday effectif $\Omega_{\alpha}(26)$ entre la lumière et le spin nucléaire hybridé par le métastable, qui contrôle le taux de compression de spin comme nous le verrons.

\section{Description quantique}

Dans la section 2, nous avons vu que l'on peut modéliser notre système physique complexe sous la forme de trois spins collectifs couplés (3) : le spin nucléaire $\vec{I}$ dans l'état fondamental, le spin $\vec{K}$ dans le niveau hyperfin $F=1 / 2$ de l'état métastable et le spin de Stokes $\vec{S}$ du champ lumineux en cavité. Dans cette section, nous présentons le traitement quantique complet de ce modèle. Après avoir introduit l'approximation de Primakoff, nous passons à la description quantique de l'échange de métastabilité qui couple les spins nucléaire et métastable.

\subsection{Approximation de Primakoff et gain métrologique dû à la compression}

Initialement, le spin nucléaire collectif $\vec{I}$, le spin collectif du métastable $\vec{K}$ et le spin de Stokes $\vec{S}$ de la lumière sont polarisés selon $O x$, et le resteront pendant toute la procédure expérimentale. Dans l'approximation de HolsteinPrimakoff, qui assimile les composantes de spin macroscopiques selon $O x$ à des variables classiques, les composantes $O y$ et $O z$ restantes, orthogonales aux spins moyens, se comportent comme les opérateurs de quadratures (parties hermitienne et antihermitienne d'opérateurs d'annihilation donc canoniquement conjuguées, $[X, P]=\mathrm{i} / 2$ ) de trois 
modes bosoniques $a, b, c:^{7}$

$$
\begin{aligned}
& \frac{I_{y}}{\sqrt{N}} \stackrel{\text { Primakoff }}{\simeq} X_{a}=\frac{a+a^{\dagger}}{2} \quad ; \quad \frac{K_{y}}{\sqrt{n}} \stackrel{\text { Primakoff }}{\simeq} X_{b}=\frac{b+b^{\dagger}}{2} \quad ; \quad \frac{S_{y}}{\sqrt{n_{\mathrm{ph}}}} \stackrel{\text { Primakoff }}{\simeq} X_{c}=\frac{c+c^{\dagger}}{2} \\
& \frac{I_{z}}{\sqrt{N}} \stackrel{\text { Primakoff }}{\simeq} P_{a}=\frac{a-a^{\dagger}}{2 \mathrm{i}} \quad ; \quad \frac{K_{z}}{\sqrt{n}} \stackrel{\text { Primakoff }}{\simeq} P_{b}=\frac{b-b^{\dagger}}{2 \mathrm{i}} \quad ; \quad \frac{S_{z}}{\sqrt{n_{\mathrm{ph}}}} \stackrel{\text { Primakoff }}{\simeq} P_{c}=\frac{c-c^{\dagger}}{2 \mathrm{i}}
\end{aligned}
$$

Nous avons tenu compte des valeurs moyennes (3) dans la normalisation. Faisons le lien avec la représentation bosonique exacte (1) du spin de Stokes en écrivant :

$$
\frac{S_{y}}{\sqrt{n_{\mathrm{ph}}}}-\mathrm{i} \frac{S_{z}}{\sqrt{n_{\mathrm{ph}}}}=\frac{1}{\sqrt{n_{\mathrm{ph}}}} c_{y}^{\dagger} c_{x} \stackrel{\text { Primakoff }}{\simeq} c_{y}^{\dagger} \quad ; \quad \frac{S_{y}}{\sqrt{n_{\mathrm{ph}}}}+\mathrm{i} \frac{S_{z}}{\sqrt{n_{\mathrm{ph}}}}=\frac{1}{\sqrt{n_{\mathrm{ph}}}} c_{x}^{\dagger} c_{y} \stackrel{\text { Primakoff }}{\simeq} c_{y}
$$

Ceci montre que l'opérateur de création $c^{\dagger}$ dans (9)-(10), identifié avec $c_{y}^{\dagger}$ dans l'approximation de Primakoff, transfère un photon du mode de cavité fortement peuplé par un état cohérent polarisé selon $O x$ dans le mode de cavité initialement vide polarisé selon $O y$. Dans l'approximation de Primakoff, l'hamiltonien du couplage de Faraday atome-champ (2) s'écrit :

$$
H=\hbar \Omega P_{b} P_{c} \quad \text { avec } \quad \Omega=\chi \sqrt{n n_{\mathrm{ph}}} .
$$

Comme $\chi$ ne dépend pas de l'intensité du champ dans la cavité, $\Omega^{2}$ est proportionnel à cette intensité. Pour finir, écrivons en termes des variables de Primakoff le paramètre $\xi$ de la référence [11] quantifiant le niveau de compression de spin utilisable dans un magnétomètre (le gain métrologique est d'autant plus élevé que $\xi$ est plus faible, voir notre note 2), $O z$ étant la direction transverse au spin moyen de plus forte compression et le spin nucléaire collectif étant de nombre quantique $I=N_{\text {cell }} / 2$ :

$$
\xi^{2} \equiv \frac{2 I \operatorname{Var}_{\text {signal }}\left(I_{z}\right)}{\left\langle I_{x}\right\rangle^{2}}=\frac{4 \operatorname{Var}_{\text {signal }}\left(P_{a}\right)}{\eta}
$$

La polarisation nucléaire $\eta$ étant fixée, et une mesure quantique non destructive étant effectuée en continu sur le spin nucléaire, il faut chercher à minimiser la variance de $P_{a}$ conditionnée au signal de mesure à définir, en la faisant descendre le plus en dessous possible de sa valeur initiale 1/4.

\section{2. Équation pilote pour l'échange de métastabilité}

Considérons dans cette sous-section l'évolution du système due au seul échange de métastabilité $(\chi=0)$. Dans un traitement quantique, les équations classiques (6)-(7) deviennent des équations stochastiques incluant les fluctuations quantiques. Dans l'approximation de Primakoff, cela donne pour les quadratures $X$ dans l'état métastable et fondamental :

$$
\mathrm{d} X_{a}=-\gamma_{f} X_{a} \mathrm{~d} t+\sqrt{\gamma_{m} \gamma_{f}} X_{b} \mathrm{~d} t+\mathrm{d} X_{a}^{\text {stoch }} \quad ; \quad \mathrm{d} X_{b}=-\gamma_{m} X_{b} \mathrm{~d} t+\sqrt{\gamma_{m} \gamma_{f}} X_{a} \mathrm{~d} t+\mathrm{d} X_{b}^{\text {stoch }}
$$

où l'on a utilisé la troisième égalité de l'équation (8). Les bruits de Langevin $\mathrm{d} X_{i}^{\text {stoch }}$, avec $i \in\{a, b\}$, ont une moyenne nulle, sont des variables aléatoires indépendantes à des temps différents et ont des variances et des covariances à temps égaux calculées dans la référence [5] :

$$
\left\langle\mathrm{d} X_{i}^{\text {stoch }} \mathrm{d} X_{j}^{\text {stoch }}\right\rangle=D_{i j} \mathrm{~d} t \quad \text { avec } \quad \underline{\underline{D}}=\frac{1}{2}\left(\begin{array}{cc}
\gamma_{f} & -\sqrt{\gamma_{m} \gamma_{f}} \\
-\sqrt{\gamma_{m} \gamma_{f}} & \gamma_{m}
\end{array}\right)
$$

On a des équations de même forme que (14) pour les quadratures $P_{i}$, avec d'autres bruits de Langevin d $P_{i}^{\text {stoch }}$, de même matrice de covariance que l'équation (15) entre eux mais de matrice de covariance avec les bruits $\mathrm{d} X_{i}^{\text {stoch }}$ donnée par

$$
\left\langle\mathrm{d} X_{i}^{\text {stoch }} \mathrm{d} P_{j}^{\text {stoch }}\right\rangle=\mathcal{D}_{i j} \mathrm{~d} t \quad \text { avec } \underline{\underline{D}}=\underline{\mathrm{i}} \underline{\underline{D}}
$$

7. Si nous considérons un grand spin $\vec{S}$ polarisé selon $O x$, nous pouvons approximer la composante de spin dans cette direction par une variable classique, en posant $\hat{S}_{x} \simeq\left\langle\hat{S}_{x}\right\rangle$ si bien que $\left[\hat{S}_{y} / \sqrt{2\left\langle\hat{S}_{x}\right\rangle}, \hat{S}_{z} / \sqrt{2\left\langle\hat{S}_{x}\right\rangle}\right] \simeq \mathrm{i} / 2$. 
Pour le calcul des valeurs moyennes et des variances des observables atomiques, cette formulation stochastique équivaut à une équation pilote sur l'opérateur densité atomique $\rho_{\text {at }}$ des deux modes bosoniques $a$ et $b$ :

$$
\frac{\mathrm{d} \rho_{\mathrm{at}}}{\mathrm{d} t}=C \rho_{\mathrm{at}} C^{\dagger}-\frac{1}{2}\left\{C^{\dagger} C, \rho_{\mathrm{at}}\right\} \quad \text { avec } \quad C=\sqrt{2 \gamma_{f}} a-\sqrt{2 \gamma_{m}} b
$$

En effet, la représentation stochastique de Langevin de l'équation pilote (17) pour un opérateur quelconque $A$ s'écrit

$$
\mathrm{d} A=\frac{\mathrm{d} t}{2}\left\{C^{\dagger}[A, C]-\left[A, C^{\dagger}\right] C\right\}+\mathrm{d} A^{\text {stoch }} \quad \text { où } \quad \mathrm{d} A^{\text {stoch }}=\left[C^{\dagger}, A\right] \mathrm{d} B+\mathrm{d} B^{\dagger}[A, C]
$$

et $\mathrm{d} B$ est un opérateur stochastique markovien de moyenne nulle, de matrice de covariance à temps égaux

$$
\left\langle\mathrm{d} B \mathrm{~d} B^{\dagger}\right\rangle=\mathrm{d} t \quad ; \quad\langle\mathrm{d} B \mathrm{~d} B\rangle=\left\langle\mathrm{d} B^{\dagger} \mathrm{d} B^{\dagger}\right\rangle=\left\langle\mathrm{d} B^{\dagger} \mathrm{d} B\right\rangle=0
$$

Pour être complets, esquissons un autre raisonnement, qui fait l'économie des bruits de Langevin quantiques. Il suffit d'admettre que les équations d'évolution sur les moyennes $\left\langle X_{i}\right\rangle$ et $\left\langle P_{i}\right\rangle$ tirées de (6)-(7) dérivent d'une équation pilote de la forme de Lindblad (52). Comme ces équations sont linéaires, les opérateurs de saut $C_{m}$ encadrant $\rho_{\text {at }}$ dans l'équation pilote sont des combinaisons linéaires de $a$ et $b$. Ceci redonne (17).

\section{3. Équation pilote à trois modes}

L'évolution complète, comprenant l'interaction atome-champ d'hamiltonien hermitien $H$ (12), l'échange de métastabilité et les pertes de la cavité, est décrite par l'équation pilote ${ }^{8}$

$$
\frac{\mathrm{d} \rho}{\mathrm{d} t}=\frac{1}{\mathrm{i} \hbar}[H, \rho]+\kappa\left(c \rho c^{\dagger}-\frac{1}{2}\left\{c^{\dagger} c, \rho\right\}\right)+C \rho C^{\dagger}-\frac{1}{2}\left\{C^{\dagger} C, \rho\right\}
$$

où $C$ est l'opérateur de saut pour l'échange de métastabilité (17), $\kappa$ est le taux de perte de la cavité, $\gamma_{m}$ et $\gamma_{f}$ sont les taux effectifs d'échange de métastabilité dans l'état métastable et fondamental.

Initialement, les trois modes sont dans l'état vide correspondant à un état polarisé pour les trois spins. Pour cet état initial, les premiers moments des quadratures restent nuls, et l'on peut obtenir un système fermé d'équations sur

8. Nous négligeons ici l'évolution interne des modes atomiques (précession de spin) en supposant que les sous-niveaux Zeeman sont dégénérés dans l'état fondamental et dans le niveau métastable $F=1 / 2$, donc que le champ magnétique extérieur est nul, $\vec{B}=\overrightarrow{0}$. Cette hypothèse simplificatrice appelle les commentaires suivants. (i) Dans l'expérience, on prévoit d'imposer un champ directeur selon $O x$ de l'ordre du $\mu \mathrm{T}$ pour éviter une précession «sauvage» du spin moyen autour d'un champ magnétique résiduel de direction inconnue [14]. D'un point de vue théorique, on se place alors dans le référentiel tournant autour de $O x$ à la pulsation de Larmor correspondante $\omega_{L}^{(x)}$ du spin nucléaire pour éliminer ce champ directeur [il faudrait en toute rigueur compenser l'écart entre les pulsations de Larmor métastable et fondamentale, par exemple au moyen d'un champ magnétique fictif créé par déplacement lumineux, mais cette précaution semble superflue car la pulsation de Larmor métastable reste petite devant le taux effectif d'échange de métastabilité $\gamma_{m}$ ]; il faut en principe faire tourner aussi la cavité à la vitesse angulaire $\omega_{L}^{(x)}$ pour qu'elle soit immobile dans le référentiel tournant; si la cavité reste fixe dans le référentiel du laboratoire, on peut établir le couplage de Faraday et effectuer une mesure du champ sortant de la cavité non plus en continu mais de manière stroboscopique (à chaque fois que la direction à comprimer en spin se confond avec l'axe optique) [7]. (ii) S'il existe pendant la phase de compression un petit champ magnétique statique parasite (en plus du champ directeur) dans le plan $y O z$, d'angle $\phi$ avec $O y$, ce champ tourne à la pulsation $-\omega_{\mathrm{L}}^{(x)}$ dans le référentiel tournant; dans l'équation stochastique du modèle à un mode (58) pour la détection homodyne, ceci ajoute une contribution d'hamiltonien hermitien $H_{\alpha}=\sqrt{N} \hbar \omega_{L}^{(y)}\left(\Omega_{\beta} / \Omega\right)\left[\cos \left(\phi-\omega_{L}^{(x)} t\right) X_{\alpha}+\sin \left(\phi-\omega_{L}^{(x)} t\right) P_{\alpha}\right]$ où $\omega_{L}^{(y)} \ll \omega_{L}^{(x)}$ est la pulsation de Larmor du spin nucléaire dans le champ parasite et $\Omega_{\beta} / \Omega$ est donné par l'équation (26). Le terme en $P_{\alpha}$ s'absorbe dans un changement de phase de l'ansatz (59). Le terme en $X_{\alpha}$ ne change pas le paramètre $u$ de l'ansatz (59) donc la variance de $P_{\alpha}$ dans une réalisation mais ajoute une partie déterministe oscillante à $\bar{P}_{\alpha}$, à savoir $\left(\sqrt{N} \omega_{L}^{(y)} / 2 \omega_{L}^{(x)}\right)\left(\Omega_{\beta} / \Omega\right)\left[\sin \left(\phi-\omega_{L}^{(x)} t\right)-\sin \phi\right]$ et, d'après $(65)$, une partie déterministe $\sqrt{\Gamma_{\text {ex }}}\left(\Omega_{\beta} / \Omega\right)\left(\sqrt{N} \omega_{L}^{(y)} / 2 \omega_{L}^{(x)}\right)\left\{\left[\cos \left(\phi-\omega_{L}^{(x)} t\right)-\cos \phi\right] /\left(\omega_{L}^{(x)} t\right)-\sin \phi\right\}$ au signal intégré $\sigma(t)$. Le terme en sin $\phi$ dans le signal peut être annulé en jouant sur l'instant initial «t $t=0$ » de la phase de compression; les autres contributions s'annulent pour une durée $t$ de l'expérience multiple entier de $2 \pi / \omega_{L}^{(x)}$. (iii) Si l'on veut utiliser l'état comprimé de spin pour mesurer un champ magnétique, on éteint la décharge et le champ directeur et on s'arrange pour que le champ à mesurer $\vec{B}_{\text {mes }}$ soit orienté selon $O y$. Le spin nucléaire collectif précesse alors dans le plan $x O z$ d'un angle qu'il faut mesurer pour remonter à $B_{\text {mes }}$, et la direction de compression initiale $O z$ est justement celle qu'il faut pour réduire l'incertitude de pointage angulaire sur le spin. Une autre stratégie consiste à partir d'un état polarisé ordinaire, non comprimé, du spin nucléaire et à effectuer, décharge allumée mais champ directeur éteint, la mesure en continu du champ lumineux sortant de la cavité en présence de $B_{\text {mes }} \vec{u}_{y}$; dans le modèle à un mode avec détection homodyne de la section 4.2.2, on retombe alors sur les propositions de magnétométrie des références [17, 18]. 
les seconds moments. On trouve que les quadratures $P$ restent de variances constantes et de covariances nulles dans les trois modes,

$$
\left\langle P_{a}^{2}\right\rangle(t)=\left\langle P_{b}^{2}\right\rangle(t)=\left\langle P_{c}^{2}\right\rangle(t)=\frac{1}{4} \quad ; \quad\left\langle P_{a} P_{b}\right\rangle(t)=\left\langle P_{a} P_{c}\right\rangle(t)=\left\langle P_{b} P_{c}\right\rangle(t)=0
$$

que la variance $\left\langle X_{c}^{2}\right\rangle$ reste bornée et que les covariances $\left\langle X_{a} X_{c}\right\rangle$ et $\left\langle X_{b} X_{c}\right\rangle$ restent nulles, tandis que les variances et la covariance des quadratures $X_{a}$ et $X_{b}$, et donc le nombre d'excitations dans les modes atomiques, ${ }^{9}$ divergent linéairement en temps, du moins tant que l'approximation de Primakoff est applicable. Nous donnons ici explicitement seulement les comportements aux temps longs :

$$
\begin{array}{ll}
\left\langle X_{a}^{2}\right\rangle(t) \underset{t \rightarrow+\infty}{=} \frac{\gamma_{m} \gamma_{f}}{\left(\gamma_{m}+\gamma_{f}\right)^{2}} \frac{\Omega^{2} t}{4 \kappa}+O(1) \quad\left\langle X_{b}^{2}\right\rangle(t) \underset{t \rightarrow+\infty}{=} \frac{\gamma_{f}^{2}}{\left(\gamma_{m}+\gamma_{f}\right)^{2}} \frac{\Omega^{2} t}{4 \kappa}+O(1) \\
\left\langle X_{a} X_{b}\right\rangle(t) \underset{t \rightarrow+\infty}{=} \frac{\gamma_{m}^{1 / 2} \gamma_{f}^{3 / 2}}{\left(\gamma_{m}+\gamma_{f}\right)^{2}} \frac{\Omega^{2} t}{4 \kappa}+O(1) \quad\left\langle X_{c}^{2}\right\rangle(t)-\frac{1}{4} \underset{t \rightarrow+\infty}{\rightarrow}\left(\frac{\Omega}{2 \kappa}\right)^{2}\left(1-\frac{2 \gamma_{m}}{\kappa+2\left(\gamma_{m}+\gamma_{f}\right)}\right)
\end{array}
$$

\subsection{Modèle à un mode}

Dans cette sous-section, nous établissons une équation pilote à un mode décrivant l'évolution lente du spin nucléaire dans la limite

$$
\Gamma_{\mathrm{ex}} \ll \gamma_{f}<\gamma_{m} \quad \text { et } \quad \Gamma_{\mathrm{ex}} \ll \kappa
$$

où $\Gamma_{\mathrm{ex}}$ est un taux de création d'excitations dans le mode bosonique nucléaire hybridé $\alpha$ défini plus bas sous l'effet du couplage de Faraday (il suffit de savoir ici que $\Gamma_{\mathrm{ex}} \propto \Omega^{2}$ si bien que (23) est une limite de couplage de Faraday faible $\Omega \rightarrow 0$ ). Il convient à cette fin d'introduire les opérateurs bosoniques d'annihilation dans une base astucieusement tournée, au moyen des combinaisons linéaires suivantes des opérateurs $a$ et $b$ :

$$
\alpha=\sqrt{\frac{\gamma_{m}}{\gamma_{m}+\gamma_{f}}} a+\sqrt{\frac{\gamma_{f}}{\gamma_{m}+\gamma_{f}}} b \quad ; \quad \beta=\sqrt{\frac{\gamma_{m}}{\gamma_{m}+\gamma_{f}}} b-\sqrt{\frac{\gamma_{f}}{\gamma_{m}+\gamma_{f}}} a
$$

$\alpha$ et $\beta$ correspondent en effet aux modes propres de la partie d'échange de métastabilité de l'équation pilote à trois modes (20) (en pratique, on a $\gamma_{m} \gg \gamma_{f}$, voir l'équation (8), si bien que le mode $\beta$ correspond au spin du métastable légèrement hybridé avec le spin de l'état fondamental, et $\alpha$ au spin nucléaire légèrement hybridé avec le spin du métastable). Tandis que le mode $\alpha$ subit une divergence en temps de son nombre moyen d'excitations (d'où la possibilité de définir un taux $\Gamma_{\mathrm{ex}}$ ), le mode $\beta$ est fortement amorti et tend vers une valeur stationnaire (voir les résultats (21) et (22), qui montrent que $\left\langle P_{\beta}^{2}\right\rangle=1 / 4$ et $\left\langle X_{\beta}^{2}\right\rangle=O(1)$ où $X_{\beta}=\left(\beta+\beta^{\dagger}\right) / 2$ ), ce qui permettra de l'éliminer adiabatiquement, tout comme le champ en cavité. Dans cette nouvelle base, l'équation pilote à trois modes (20) prend la forme

$$
\frac{\mathrm{d} \rho}{\mathrm{d} t}=\frac{1}{\mathrm{i} \hbar}[H, \rho]+\kappa\left(c \rho c^{\dagger}-\frac{1}{2}\left\{c^{\dagger} c, \rho\right\}\right)+\gamma_{\beta}\left(\beta \rho \beta^{\dagger}-\frac{1}{2}\left\{\beta^{\dagger} \beta, \rho\right\}\right)
$$

où $\gamma_{\beta} \equiv 2\left(\gamma_{m}+\gamma_{f}\right)$ et, en notant $P_{\alpha}=\left(\alpha-\alpha^{\dagger}\right) / 2$ i et $P_{\beta}=\left(\beta-\beta^{\dagger}\right) / 2 \mathrm{i}$ les quadratures $P$ des nouveaux modes,

$$
H=\hbar\left(\Omega_{\alpha} P_{\alpha}+\Omega_{\beta} P_{\beta}\right) P_{c} \quad \text { avec } \quad \Omega_{\alpha} \equiv \Omega \sqrt{\frac{\gamma_{f}}{\gamma_{m}+\gamma_{f}}} \quad \text { et } \quad \Omega_{\beta} \equiv \Omega \sqrt{\frac{\gamma_{m}}{\gamma_{m}+\gamma_{f}}}
$$

La référence [25] explique en toute généralité comment effectuer une élimination adiabatique au niveau de l'équation pilote. Ici, nous préférons l'effectuer, comme dans la référence [26], en couplage de Faraday faible $\Omega \rightarrow 0$ dans le formalisme des fonctions d'onde Monte-Carlo [27, 28], où l'opérateur densité solution de l'équation pilote (25)

9. Pour l'état initial considéré, on a à tout temps $\left\langle X_{a}\right\rangle=0$ et $\left\langle X_{a}^{2}\right\rangle-\frac{1}{4}=\left\langle a^{\dagger} a\right\rangle$, où $\left\langle a^{\dagger} a\right\rangle$ est le nombre moyen d'excitations dans le mode de spin nucléaire, si bien que $\operatorname{Var} X_{a}=\left\langle a^{\dagger} a\right\rangle+\frac{1}{4}$; en effet, on a $a^{\dagger} a+1 / 2=X_{a}^{2}+P_{a}^{2}$. Les mêmes relations valent pour les deux autres modes. 
s'obtient par moyenne de cas purs sur des réalisations stochastiques indépendantes, chaque réalisation correspondant à l'évolution déterministe d'un vecteur d'état non normalisé $|\psi(t)\rangle$ sous l'action de l'hamiltonien effectif non hermitien

$$
H_{\mathrm{eff}}=H-\frac{\mathrm{i} \hbar}{2}\left(\kappa c^{\dagger} c+\gamma_{\beta} \beta^{\dagger} \beta\right)
$$

interrompue aléatoirement par des sauts quantiques (évolutions discontinues $|\psi\rangle \rightarrow C|\psi\rangle$ ) d'opérateurs de saut

$$
C_{c}=\sqrt{\kappa} c \text { et } \quad C_{\beta}=\sqrt{\gamma_{\beta}} \beta .
$$

En l'absence du couplage cohérent $\Omega$ dans (26) le mode métastable hybridé et le mode de cavité restent dans l'état vide initial. Au premier ordre en $\Omega$, cet état est couplé à des états à une excitation dans la cavité (par l'action de $P_{c}$ ) et à zéro ou une excitation dans le mode du métastable hybridé (par l'action de $P_{\alpha}$ ou de $P_{\beta}$ ). Nous pouvons alors tronquer le vecteur d'état Monte-Carlo $|\psi\rangle$ dans la base de Fock $\left\{\left|n_{\alpha}\right\rangle_{\text {fond }}\left|n_{\beta}\right\rangle_{\text {méta }}\left|n_{c}\right\rangle_{\text {cav }}\right\}$ comme suit,

$$
|\psi\rangle=\left|\psi_{\alpha}^{00}\right\rangle|0\rangle|0\rangle+\left|\psi_{\alpha}^{01}\right\rangle|0\rangle|1\rangle+\left|\psi_{\alpha}^{11}\right\rangle|1\rangle|1\rangle
$$

en commettant une erreur de norme $O\left(\Omega^{2}\right)$. Sous l'effet de l'hamiltonien effectif (27), les composantes rapides $\left|\psi_{\alpha}^{01}\right\rangle$ et $\left|\psi_{\alpha}^{11}\right\rangle$ rejoignent exponentiellement un régime de suivi adiabatique de la composante lente $\left|\psi_{\alpha}^{00}\right\rangle$ avec des taux $\kappa / 2$ ou $\left(\kappa+\gamma_{\beta}\right) / 2$. D'où leur élimination adiabatique dans la limite $(23)^{10}$

$$
\left|\psi_{\alpha}^{11}\right\rangle_{\text {adiab }} \simeq \frac{\mathrm{i} \Omega_{\beta}}{2\left(\kappa+\gamma_{\beta}\right)}\left|\psi_{\alpha}^{00}\right\rangle \quad \text { et } \quad\left|\psi_{\alpha}^{01}\right\rangle_{\text {adiab }} \simeq \frac{\Omega_{\alpha}}{\kappa} P_{\alpha}\left|\psi_{\alpha}^{00}\right\rangle
$$

On reporte les expressions de $\left|\psi_{\alpha}^{11}\right\rangle_{\text {adiab }},\left|\psi_{\alpha}^{01}\right\rangle_{\text {adiab }}$ dans l'équation d'évolution hamiltonienne de $\left|\psi_{\alpha}^{00}\right\rangle$ pour obtenir

$$
\mathrm{i} \hbar \frac{\mathrm{d}}{\mathrm{d} t}\left|\psi_{\alpha}^{00}\right\rangle=-\frac{\mathrm{i} \hbar}{2}\left(\Gamma_{\mathrm{ex}} P_{\alpha}^{2}+\Gamma_{0}\right)\left|\psi_{\alpha}^{00}\right\rangle \equiv H_{\mathrm{eff}}^{00}\left|\psi_{\alpha}^{00}\right\rangle
$$

où l'on a introduit les taux

$$
\Gamma_{\mathrm{ex}}=\frac{\Omega_{\alpha}^{2}}{\kappa} \quad \text { et } \quad \Gamma_{0}=\frac{\Omega_{\beta}^{2}}{4\left(\kappa+\gamma_{\beta}\right)}
$$

En étudiant l'effet de l'opérateur de saut de cavité $C_{c}$ et de saut d'échange de métastabilité $C_{\beta}$ sur le vecteur d'état (29), nous pouvons interpréter l'hamiltonien effectif de l'équation (31). (i) Considérons d'abord l'effet d'un saut de cavité, qui se produit à l'instant $t$ avec un taux $\kappa\left(\left\langle\psi_{\alpha}^{11} \mid \psi_{\alpha}^{11}\right\rangle+\left\langle\psi_{\alpha}^{01} \mid \psi_{\alpha}^{01}\right\rangle\right)_{\text {adiab }} /\left\langle\psi_{\alpha}^{00} \mid \psi_{\alpha}^{00}\right\rangle$. Juste après le saut, le vecteur d'état, initialement en régime de suivi adiabatique, devient

$$
\left|\psi\left(t^{+}\right)\right\rangle=C_{c}\left|\psi\left(t^{-}\right)\right\rangle_{\text {adiab }} \propto\left|\psi_{\alpha}^{01}\left(t^{-}\right)\right\rangle_{\text {adiab }}|0\rangle|0\rangle+\left|\psi_{\alpha}^{11}\left(t^{-}\right)\right\rangle_{\text {adiab }}|1\rangle|0\rangle
$$

C'est la superposition d'une composante instable $|1\rangle|0\rangle$ et d'une composante stable $|0\rangle|0\rangle$. Avec une probabilité $\left\langle\psi_{\alpha}^{11} \mid \psi_{\alpha}^{11}\right\rangle_{\text {adiab }} /\left(\left\langle\psi_{\alpha}^{01} \mid \psi_{\alpha}^{01}\right\rangle+\left\langle\psi_{\alpha}^{11} \mid \psi_{\alpha}^{11}\right\rangle\right)_{\text {adiab }}$ le saut de cavité est alors suivi d'un saut d'échange de métastabilité avant

10. Dans le suivi adiabatique, les probabilités d'occupation des composantes excitées sont $\left\langle\psi_{\alpha}^{11} \mid \psi_{\alpha}^{11}\right\rangle_{\text {adiab }} /\langle\psi \mid \psi\rangle=\left[\Omega_{\beta}^{2} / 4\left(\kappa+\gamma_{\beta}\right)^{2}\right]\left\langle\psi_{\alpha}^{00} \mid \psi_{\alpha}^{00}\right\rangle /\langle\psi \mid \psi\rangle$ et $\left\langle\psi_{\alpha}^{01} \mid \psi_{\alpha}^{01}\right\rangle_{\text {adiab }} /\langle\psi \mid \psi\rangle=\left(\Gamma_{\text {ex }} / \kappa\right)\left\langle\psi_{\alpha}^{00}\left|P_{\alpha}^{2}\right| \psi_{\alpha}^{00}\right\rangle /\langle\psi \mid \psi\rangle$ où l'on a utilisé (32). Dans la limite (23), on vérifie aisément qu'elles sont $\ll 1$, si bien que presque toute la population est dans la composante $\left|\psi_{\alpha}^{00}\right\rangle|0\rangle|0\rangle$ comme il se doit, ce qui nous permettra dans la suite de remplacer $\langle\psi \mid \psi\rangle$ par $\left\langle\psi_{\alpha}^{00} \mid \psi_{\alpha}^{00}\right\rangle$. On vérifie également qu'une autre condition de validité de l'élimination adiabatique, à savoir la lenteur de l'évolution du spin nucléaire hybridé $\alpha$ par rapport aux variables rapides, qui s'écrit ici $\Gamma_{\mathrm{ex}}, \Gamma_{0} \ll \kappa, \kappa+\gamma_{\beta}$, est satisfaite. Ces considérations ne permettent cependant pas de montrer que la condition $\Gamma_{\mathrm{ex}} \ll \gamma_{f}$ est nécessaire (sauf si $\kappa \ll \gamma_{\beta}$ ). Pour le voir en toute généralité, nous poussons à l'ordre $\Omega^{4}$ le calcul de l'hamiltonien effectif $H_{\mathrm{eff}}^{00}=P H_{\mathrm{eff}} P+P H Q\left(z Q-Q H_{\mathrm{eff}} Q\right)^{-1} Q H P$ dans le sous-espace $n_{\beta}=n_{c}=0$ sur lequel $P$ projette (ici $Q=\mathbb{1}-P$ et $z=O\left(\Omega^{2}\right)$ ). Qualitativement, à cet ordre, par action de $H_{\alpha}$ puis $H_{\beta}$ sur $\left|\psi_{\alpha}^{00}\right\rangle|0\rangle|0\rangle$ (avec la notation évidente $H=H_{\alpha}+H_{\beta}$ ), on crée virtuellement une excitation $\beta$ seule, relaxant au taux $\gamma_{\beta} / 2$, d'où la condition d'adiabaticité supplémentaire $\Gamma_{0} \ll \gamma_{\beta}$; jointe à $\Gamma_{0} \ll \kappa$ et $\gamma_{f}<\gamma_{m}$, elle implique $\Gamma_{\text {ex }} \ll \gamma_{f}$ puisque $\Gamma_{\mathrm{ex}} / \gamma_{f}=\left(\Gamma_{0} / \kappa+\Gamma_{0} / \gamma_{\beta}\right)\left(4 \gamma_{\beta} / \gamma_{m}\right)<16\left(\Gamma_{0} / \kappa+\Gamma_{0} / \gamma_{\beta}\right)$. Quantitativement, nous trouvons une correction au coefficient de $P_{\alpha}^{2}$ dans $H_{\mathrm{eff}}^{00}$ de type $H_{\alpha} G_{0} H_{\beta} G_{0} H_{\beta} G_{0} H_{\alpha}\left(G_{0}\right.$ est la résolvante de $H_{\text {eff }}$ pour $\left.\Omega=0\right)$ de la forme $\hbar \Gamma_{\text {ex }} \Omega_{\beta}^{2} / \gamma_{\beta} \kappa$, qui doit être négligeable, ce qui impose $\Omega_{\beta}^{2} / \gamma_{\beta} \kappa \ll 1$, c'est-à-dire $\Gamma_{\mathrm{ex}} \ll \gamma_{f}$ compte tenu de $\gamma_{f}<\gamma_{m}$. Les corrections au terme scalaire sont négligeables dès que $\Gamma_{0} \ll \gamma_{\beta}, \kappa$, et le nouveau terme en $P_{\alpha}^{4}$ qui apparaît est négligeable devant $\hbar \Gamma_{\mathrm{ex}} P_{\alpha}^{2}$ pour $P_{\alpha}=O(1)$ si $\Gamma_{\mathrm{ex}} \ll \kappa$. 
que le vecteur d'état du système n'ait le temps de rejoindre sa valeur adiabatique. On a dans ce cas un « saut double», qui en définitive n'affecte pas la composante $\left|\psi_{\alpha}^{00}\left(t^{-}\right)\right\rangle$puisque

$$
C_{\beta} C_{c}\left|\psi\left(t^{-}\right)\right\rangle_{\text {adiab }} \propto\left|\psi_{\alpha}^{00}\left(t^{-}\right)\right\rangle|0\rangle|0\rangle
$$

Ce processus contribue au terme scalaire (proportionnel à l'identité) dans l'hamiltonien effectif de l'équation (31). Avec la probabilité complémentaire $\left\langle\psi_{\alpha}^{01} \mid \psi_{\alpha}^{01}\right\rangle_{\text {adiab }} /\left(\left\langle\psi_{\alpha}^{01} \mid \psi_{\alpha}^{01}\right\rangle+\left\langle\psi_{\alpha}^{11} \mid \psi_{\alpha}^{11}\right\rangle\right)_{\text {adiab }}$ le vecteur d'état rejoint sa valeur adiabatique avant que d'autres sauts ne se produisent, et est asservi à $\left|\psi_{\alpha}^{(01)}\left(t^{-}\right)\right\rangle_{\text {adiab }} \propto P_{\alpha}\left|\psi_{\alpha}^{00}\left(t^{-}\right)\right\rangle$, c'est-à-dire que la composante lente $\left|\psi_{\alpha}^{00}\left(t^{-}\right)\right\rangle$a subi de manière effective un saut quantique simple avec un opérateur de saut proportionnel à $P_{\alpha}$. Ce processus correspond au premier terme, proportionnel à $P_{\alpha}^{2}$, dans l'hamiltonien effectif de l'équation (31). (ii) Supposons ensuite que le saut à l'instant $t$ est un saut d'échange de métastabilité, ce qui se produit avec un taux $\gamma_{\beta}\left\langle\psi_{\alpha}^{11} \mid \psi_{\alpha}^{11}\right\rangle_{\text {adiab }} /\left\langle\psi_{\alpha}^{00} \mid \psi_{\alpha}^{00}\right\rangle$. On vérifie dans ce cas que le vecteur d'état après le saut, $C_{\beta}\left|\psi\left(t^{-}\right)\right\rangle$, est entièrement instable et subit presque immédiatement un second saut, un saut de cavité. L'effet total correspond là encore à un saut double et à l'action d'un opérateur scalaire sur la composante lente. Nous tirons de cette discussion les taux de saut simple et de saut double suivants :

$$
\begin{aligned}
\Gamma_{s} & =\frac{\kappa\left(\left\langle\psi_{\alpha}^{11} \mid \psi_{\alpha}^{11}\right\rangle+\left\langle\psi_{\alpha}^{01} \mid \psi_{\alpha}^{01}\right\rangle\right)_{\text {adiab }}}{\left\langle\psi_{\alpha}^{00} \mid \psi_{\alpha}^{00}\right\rangle} \frac{\left\langle\psi_{\alpha}^{01} \mid \psi_{\alpha}^{01}\right\rangle_{\text {adiab }}}{\left(\left\langle\psi_{\alpha}^{01} \mid \psi_{\alpha}^{01}\right\rangle+\left\langle\psi_{\alpha}^{11} \mid \psi_{\alpha}^{11}\right\rangle\right)_{\text {adiab }}}=\Gamma_{\text {ex }} \frac{\left\langle\psi_{\alpha}^{00}\left|P_{\alpha}^{2}\right| \psi_{\alpha}^{00}\right\rangle}{\left\langle\psi_{\alpha}^{00} \mid \psi_{\alpha}^{00}\right\rangle} \equiv \Gamma_{\mathrm{ex}}\left\langle P_{\alpha}^{2}\right\rangle \\
\Gamma_{d} & =\frac{\kappa\left(\left\langle\psi_{\alpha}^{11} \mid \psi_{\alpha}^{11}\right\rangle+\left\langle\psi_{\alpha}^{01} \mid \psi_{\alpha}^{01}\right\rangle\right)_{\text {adiab }}}{\left\langle\psi_{\alpha}^{00} \mid \psi_{\alpha}^{00}\right\rangle} \frac{\left\langle\psi_{\alpha}^{11} \mid \psi_{\alpha}^{11}\right\rangle_{\text {adiab }}}{\left(\left\langle\psi_{\alpha}^{01} \mid \psi_{\alpha}^{01}\right\rangle+\left\langle\psi_{\alpha}^{11} \mid \psi_{\alpha}^{11}\right\rangle\right)_{\text {adiab }}}+\frac{\gamma_{\beta}\left\langle\psi_{\alpha}^{11} \mid \psi_{\alpha}^{11}\right\rangle_{\text {adiab }}}{\left\langle\psi_{\alpha}^{00} \mid \psi_{\alpha}^{00}\right\rangle}=\Gamma_{0}
\end{aligned}
$$

On obtient finalement l'équation pilote à un mode décrivant l'évolution lente de l'opérateur densité $\rho_{\alpha}$ du mode bosonique $\alpha$ (hybridé mais presque purement de spin nucléaire) :

$$
\frac{\mathrm{d} \rho_{\alpha}}{\mathrm{d} t}=C_{s} \rho_{\alpha} C_{s}^{\dagger}-\frac{1}{2}\left\{C_{s}^{\dagger} C_{s}, \rho_{\alpha}\right\}+C_{d} \rho_{\alpha} C_{d}^{\dagger}-\frac{1}{2}\left\{C_{d}^{\dagger} C_{d}, \rho_{\alpha}\right\}
$$

en termes de deux sauts quantiques, le saut simple (uniquement de cavité) $C_{s}$ et le saut double (de cavité et d'échange de métastabilité dans cet ordre ou dans l'autre) $C_{d}$ :

$$
C_{s}=\sqrt{\Gamma_{\mathrm{ex}}} P_{\alpha} \quad ; \quad C_{d}=\sqrt{\Gamma_{0}} \mathbb{1}
$$

De l'équation (37) intégrée pour l'état initial vide de $\alpha$, on tire :

$$
\left\langle X_{\alpha}^{2}\right\rangle=\frac{1}{4}\left(1+\Gamma_{\mathrm{ex}} t\right) \quad ; \quad\left\langle P_{\alpha}^{2}\right\rangle=\frac{1}{4}
$$

ce qui désigne effectivement $\Gamma_{\mathrm{ex}}$ comme un taux de création d'excitations dans le mode $\alpha$. En revenant à la base atomique initiale (non tournée) et en limitant le vecteur d'état (29) à son premier terme, on retrouve l'équation (21) et les trois premiers résultats de l'équation (22) du modèle à trois modes, pourtant valables à couplage de Faraday $\Omega$ quelconque, pas nécessairement infinitésimal. Enfin, le nombre moyen de photons polarisés selon $O y$ sortant de la cavité par unité de temps, donné dans le modèle à un mode par $\Gamma_{0}+\Gamma_{\mathrm{ex}} / 4$ comme le montrera l'équation (45), est en accord avec la valeur exacte $\kappa\left\langle c^{\dagger} c\right\rangle_{s}$ où le nombre moyen stationnaire de photons polarisés selon $O y$ dans la cavité $\left\langle c^{\dagger} c\right\rangle_{s}=\left\langle X_{c}^{2}\right\rangle_{s}-1 / 4$ est le dernier résultat de (22). ${ }^{11}$

\section{Mesure quantique non destructive du spin nucléaire en continu}

Les moyennes quantiques calculées dans la section 3 correspondent seulement aux moyennes d'ensemble sur un nombre infini de réalisations de l'expérience. Dans cette section, nous étudions ce qui nous intéresse vraiment,

11. En revanche, la valeur de $\left\langle c^{\dagger} c\right\rangle_{\text {adiab }}$ dans la forme adiabatique (30) du vecteur d'état ne représente pas ce nombre. La solution du paradoxe tient à l'existence de la voie de désexcitation (ii), celle de l'annihilation en premier saut de l'excitation $n_{\beta}=1$ dans le mode du métastable immédiatement suivie par la perte d'un photon en cavité. Le vrai taux de sortie de photons polarisés selon $O y$ est donc $\kappa\left\langle c^{\dagger} c\right\rangle_{\text {adiab }}+\gamma_{\beta}\left\langle\beta^{\dagger} \beta\right\rangle_{\text {adiab }}$ 
l'évolution du système dans une ou plusieurs réalisations données, conditionnée aux résultats d'une mesure en continu sur la lumière polarisée selon $O y$ sortant de la cavité. Pour cela, nous revenons à la formulation en termes de fonctions d'onde Monte-Carlo, comme dans la section 3, où des trajectoires stochastiques $|\psi(t)\rangle$ correspondant à une succession particulière de sauts quantiques reconstruisent l'opérateur densité du système conditionné à des résultats de mesure [28]. La forme précise des opérateurs de saut Monte-Carlo, qui n'est pas unique dans la reformulation stochastique d'une équation pilote, est alors déterminée par les mesures particulières effectuées.

\section{1. Étude de la compression par comptage de photons : résultats analytiques à un mode et numériques à trois modes}

Supposons que l'on compte en continu et directement (par photodétection) le nombre de photons polarisés selon Oy sortant de la cavité (voir la figure 1b), comme l'a proposé la référence [29]. L'opérateur de saut associé à cette mesure est $\sqrt{\kappa} c$, de sorte que l'équation pilote à trois modes (20) est déjà sous la bonne forme pour analyser l'évolution du vecteur d'état $|\psi(t)\rangle$ conditionnée à la mesure.

Il en va de même dans la limite d'un faible couplage de Faraday, $\Omega \rightarrow 0$, qui conduit au modèle à un mode de la section 3.4. Comme les opérateurs de saut $C_{d}$ et $C_{s}$ de son équation pilote (37) correspondent tous deux à la perte en cavité d'un photon polarisé selon $O y$ (rappelons-le, $C_{d}$ résulte d'un saut de cavité immédiatement suivi ou précédé d'un saut d'échange de métastabilité, et $C_{s}$ d'un saut simple de cavité), la mesure ne peut faire la distinction entre les deux, et l'opérateur densité conditionné à un nombre donné $n$ de photons détectés est obtenu en moyennant sur des réalisations ayant ce même nombre total $n$ de sauts. Un vecteur d'état Monte-Carlo non normalisé ayant subi ces $n$ sauts pendant la durée $t$ s'écrit

$$
|\psi(t)\rangle=\mathrm{e}^{-\frac{\mathrm{i}}{\hbar} H_{\mathrm{eff}}^{00}\left(t-t_{n}\right)} C_{\epsilon_{n}} \mathrm{e}^{-\frac{\mathrm{i}}{\hbar} H_{\mathrm{eff}}^{00}\left(t_{n}-t_{n-1}\right)} C_{\epsilon_{n-1}} \ldots C_{\epsilon_{1}} \mathrm{e}^{-\frac{\mathrm{i}}{\hbar} H_{\mathrm{eff}}^{00} t_{1}}|\psi(0)\rangle
$$

où $\epsilon_{k} \in\{s, d\}$ et $t_{k}$ sont le type et l'instant du kème saut, $H_{\text {eff }}^{00}$ est l'hamiltonien effectif (31) et nous notons le vecteur d'état du modèle à un mode $|\psi\rangle$ plutôt que $\left|\psi_{\alpha}^{00}\right\rangle$ pour alléger. La moyenne quantique d'une observable $O$ s'obtient en moyennant sur toutes les trajectoires possibles, donc en sommant sur le nombre et le type des sauts et en intégrant sur leurs instants :

$$
\langle O\rangle(t)=\sum_{n} \int_{0<t_{1}<t_{2} \ldots<t_{n}<t} \mathrm{~d} t_{1} \mathrm{~d} t_{2} \ldots \mathrm{d} t_{n} \sum_{\left(\epsilon_{k}\right)}\langle\psi(t)|O| \psi(t)\rangle
$$

où la norme au carré de chaque vecteur d'état non normalisé $|\psi(t)\rangle$ donne automatiquement sa densité de probabilité [30]. En prenant $O=\mathbb{1}$, nous en déduisons la probabilité que $n$ sauts se soient produits dans l'intervalle de temps $[0, t]$ :

$$
\Pi_{n}(t)=\int_{0<t_{1}<t_{2} \ldots<t_{n}<t} \mathrm{~d} t_{1} \mathrm{~d} t_{2} \ldots \mathrm{d} t_{n} \sum_{\left(\epsilon_{k}\right)_{1 \leq k \leq n} \in\{s, d\}^{n}}\langle\psi(t) \mid \psi(t)\rangle
$$

Pour évaluer (42), nous tirons parti du fait que tous les opérateurs de saut dans (40) et leurs conjugués hermitiens commutent entre eux et avec $H_{\mathrm{eff}}^{00}{ }^{12}$ En utilisant les identités

$$
\sum_{\epsilon_{n}=s, d} \ldots \sum_{\epsilon_{1}=s, d}\left(C_{\epsilon_{n}}^{\dagger} C_{\epsilon_{n}} \ldots C_{\epsilon_{1}}^{\dagger} C_{\epsilon_{1}}\right)=\left(\sum_{\epsilon_{n}=s, d} C_{\epsilon_{n}}^{\dagger} C_{\epsilon_{n}}\right) \ldots\left(\sum_{\epsilon_{n}=s, d} C_{\epsilon_{1}}^{\dagger} C_{\epsilon_{1}}\right)=\left(\Gamma_{\mathrm{ex}} P_{\alpha}^{2}+\Gamma_{0} \mathbb{1}\right)^{n}
$$

et en injectant une relation de fermeture dans la base propre de $P_{\alpha}$ telle que $P_{\alpha}\left|p_{\alpha}\right\rangle=p_{\alpha}\left|p_{\alpha}\right\rangle$, après avoir intégré sur les temps $t_{k}$ comme le permet le produit télescopique des opérateurs d'évolution, nous obtenons

$$
\Pi_{n}(t)=\frac{t^{n}}{n !} \int_{-\infty}^{+\infty} \mathrm{d} p_{\alpha}\left(\Gamma_{\mathrm{ex}} p_{\alpha}^{2}+\Gamma_{0}\right)^{n} \mathrm{e}^{-\Gamma_{\mathrm{ex}} p_{\alpha}^{2} t} \mathrm{e}^{-\Gamma_{0} t} \Pi\left(p_{\alpha}, 0\right)=\left(\begin{array}{c}
2 n \\
n
\end{array}\right) \frac{\left(\Gamma_{\mathrm{ex}} t / 8\right)^{n} \mathrm{e}^{-\Gamma_{0} t}}{\left(1+\Gamma_{\mathrm{ex}} t / 2\right)^{n+1 / 2}} \Phi\left(-n, \frac{1}{2}-n ; \Gamma_{0} t+\frac{2 \Gamma_{0}}{\Gamma_{\mathrm{ex}}}\right)
$$

où $\Pi\left(p_{\alpha}, 0\right)$ est la distribution de probabilité initiale de $p_{\alpha}$ (une gaussienne de moyenne nulle et de variance $1 / 4$ ) et $\Phi$ est la fonction hypergéométrique confluente de Kummer ${ }_{1} F_{1}$. On remarque que (44) est en fait une moyenne

12. Pour cette raison, garder l'information sur les instants des sauts ne permet pas, par post-sélection, d'augmenter l'efficacité de la compression de spin. En effet, l'opérateur densité $\left.\rho_{\alpha}(t)\right|_{t_{1}, \ldots, t_{n}}$ sachant que $n$ sauts se sont produits aux instants $t_{1}, \ldots, t_{n}$ conduit à la même distribution de probabilité de $P_{\alpha}$ que l'opérateur densité $\left.\rho_{\alpha}(t)\right|_{n}$ sachant seulement qu'il y a eu $n$ sauts pendant $[0, t]$. 
gaussienne sur $p_{\alpha}$ d'une loi de Poisson de paramètre $\lambda=\left(\Gamma_{\mathrm{ex}} p_{\alpha}^{2}+\Gamma_{0}\right) t$. On en déduit la moyenne et la variance du nombre de photodétections pendant la durée $t$ :

$$
\langle n\rangle=\left(\Gamma_{0}+\frac{1}{4} \Gamma_{\mathrm{ex}}\right) t \quad ; \quad \operatorname{Var} n=\langle n\rangle+\frac{\left(\Gamma_{\mathrm{ex}} t\right)^{2}}{8}
$$

Toujours en utilisant l'équation (44), nous accédons à la distribution de probabilité de $p_{\alpha}$ sachant que $n$ photons ont été détectés dans l'intervalle de temps $[0, t]$ :

$$
\Pi_{t}\left(p_{\alpha} \mid n\right)=\frac{1}{\Pi_{n}(t)} \frac{t^{n}}{n !}\left(\Gamma_{\mathrm{ex}} p_{\alpha}^{2}+\Gamma_{0}\right)^{n} \mathrm{e}^{-\Gamma_{\mathrm{ex}} p_{\alpha}^{2} t} \mathrm{e}^{-\Gamma_{0} t} \Pi\left(p_{\alpha}, 0\right)
$$

Comme on pouvait s'y attendre, c'est une fonction paire de $p_{\alpha}$, la photodétection donnant accès seulement à l'intensité du champ sortant polarisé selon $O y$ et ne pouvant distinguer entre des valeurs opposées $\pm p_{\alpha}$ de la quadrature $P_{\alpha}$ du spin nucléaire hybridé selon $O z$. Il en résulte donc une compression des fluctuations de $P_{\alpha}^{2}$ plutôt que de $P_{\alpha}$, ce que nous caractérisons par la moyenne et la variance conditionnelles de $P_{\alpha}^{2}$ sachant que $n$ photons ont été détectés pendant $t$, déduites de (46) :

$$
\left\langle P_{\alpha}^{2}\right\rangle_{n}=\frac{(n+1)}{\Gamma_{\mathrm{ex}} t} \frac{\Pi_{n+1}(t)}{\Pi_{n}(t)}-\frac{\Gamma_{0}}{\Gamma_{\mathrm{ex}}} \quad ; \quad \operatorname{Var}_{n}\left(P_{\alpha}^{2}\right) \equiv\left\langle P_{\alpha}^{4}\right\rangle_{n}-\left\langle P_{\alpha}^{2}\right\rangle_{n}^{2}=\frac{(n+1)^{2}}{\left(\Gamma_{\mathrm{ex}} t\right)^{2}}\left[\frac{(n+2) \Pi_{n+2}(t)}{(n+1) \Pi_{n}(t)}-\frac{\Pi_{n+1}^{2}(t)}{\Pi_{n}^{2}(t)}\right]
$$

Enfin, au moyen de l'équation (46), nous trouvons que pour $\Gamma_{\mathrm{ex}} t \rightarrow+\infty$, la distribution de probabilité de $p_{\alpha}^{2}$ conditionnée au nombre $n$ de photodétections est piquée autour d'une valeur $p_{0}^{2}$ avec une variance conditionnelle tendant vers zéro $^{13}$ :

$$
p_{0}^{2}-\frac{1}{4}=\frac{n-\langle n\rangle}{\Gamma_{\mathrm{ex}} t} \quad \text { d'où }\left\langle P_{\alpha}^{2}\right\rangle_{n} \underset{\Gamma_{\mathrm{ex}} t \rightarrow+\infty}{\sim} p_{0}^{2} ; \quad \operatorname{Var}_{n}\left(P_{\alpha}^{2}\right) \underset{\Gamma_{\mathrm{ex}} t \rightarrow+\infty}{\sim} \frac{n}{\left(\Gamma_{\mathrm{ex}} t\right)^{2}} \rightarrow 0
$$

En remplaçant dans cette expression $n$ par sa valeur moyenne et en tenant compte de la valeur $1 / 8$ de la variance de $P_{\alpha}^{2}$ dans l'état initial, nous aboutissons au taux de compression du spin nucléaire par comptage de photons :

$$
\Gamma_{\mathrm{sq}}=\frac{\Gamma_{\mathrm{ex}}^{2}}{8\left(\Gamma_{0}+\frac{1}{4} \Gamma_{\mathrm{ex}}\right)}
$$

De même, la distribution de probabilité conditionnelle de $p_{\alpha}$ présente deux pics à $\pm p_{0}$ comme on peut le voir sur la fonction de Wigner de la figure $5 \mathrm{~b}$, obtenue par simulation numérique de l'évolution conditionnelle du système aux temps longs dans le modèle à un mode (37). ${ }^{14}$ Ceci montre bien que, lors d'une réalisation donnée de l'expérience, la

13. D'après l'équation (45), le second membre de la première équation dans (48) est asymptotiquement de l'ordre de l'unité pour une séquence de photodétection typique. Cette équation n'a en fait de sens que pour $p_{0}^{2}$ positif donc $n>\Gamma_{0} t$; alors, les équivalents (48) s'appliquent lorsque l'écart entre les deux pics dans $\Pi_{t}\left(p_{\alpha} \mid n\right)$ est beaucoup plus grand que leur largeur, ce qui impose $2 p_{0}^{2} \gg n^{1 / 2} / \Gamma_{\mathrm{ex}} t=\left(\Gamma_{0}+\Gamma_{\mathrm{ex}} p_{0}^{2}\right)^{1 / 2} / \Gamma_{\mathrm{ex}} t^{1 / 2}$. Pour les obtenir, on pose $n=\gamma t$ avec $\gamma>\Gamma_{0}$, puis on écrit (46) sous la forme $\exp \left[-t S\left(p_{\alpha}\right)\right] \Pi\left(p_{\alpha}, 0\right)$ et on quadratise $S\left(p_{\alpha}\right)$ autour de ses minima.

14. L'absence de franges montre qu'on a préparé un mélange statistique plutôt qu'une superposition cohérente de deux états comprimés en la quadrature $P_{\alpha}$. On trouve en effet à partir de l'équation (41) que $\left\langle p_{0}\left|\rho_{n}(t)\right|-p_{0}\right\rangle /\left\langle p_{0}\left|\rho_{n}(t)\right| p_{0}\right\rangle=\left[\left(\Gamma_{0}-\Gamma_{\operatorname{ex}} p_{0}^{2}\right) /\left(\Gamma_{0}+\Gamma_{\text {ex }} p_{0}^{2}\right)\right]^{n} \simeq \exp \left(-2 \Gamma_{\operatorname{ex}} t p_{0}^{2}\right) \lll 1$ sur la figure $5 \mathrm{~b}, \rho_{n}(t)$ étant l'opérateur densité conditionnel. La méthode de Laplace donne aux temps longs la distribution de Wigner conditionnelle $W_{t}\left(x_{\alpha}, p_{\alpha} \mid n\right) \sim \pi \Pi_{t}\left(p_{\alpha} \mid n\right) \exp \left[-2 x_{\alpha}^{2} / \Gamma_{\mathrm{ex}} t s\left(p_{\alpha}\right)\right] / \sqrt{\pi \Gamma_{\mathrm{ex}} t s\left(p_{\alpha}\right) / 2}$ avec $s\left(p_{\alpha}\right)=\left[1+\left(\Gamma_{0}+\Gamma_{\mathrm{ex}} p_{0}^{2}\right) /\left(\Gamma_{0}+\Gamma_{\mathrm{ex}} p_{\alpha}^{2}\right)\right] / 2$. On remarque que $s\left( \pm p_{0}\right)=$ 1 et $s\left(p_{\alpha}\right) \simeq 1$ pour $\Gamma_{0} \gg \Gamma_{\text {ex }}$. Alors que $W_{t}(0,0 \mid n)$ est écrasé exponentiellement en temps (dans la limite $\Gamma_{\text {ex }} \ll \Gamma_{0}$, il vient $W_{t}(0,0 \mid n) \sim$ $\left.\left(\Gamma_{\mathrm{ex}} / \Gamma_{0}\right)^{1 / 2} p_{0} \exp \left(2 p_{0}^{2}\right) \exp \left(-4 p_{0}^{4} \Gamma_{\mathrm{sq}} t\right)\right)$, on a aussi $W_{t}(0,0 \mid n)=\langle 2 \varepsilon\rangle_{n}$ où $\varepsilon= \pm 1$ est la parité de la fonction d'onde Monte-Carlo $\psi\left(p_{\alpha}, t\right)$. Dans une simulation numérique, on a donc seulement une décroissance lente $\langle\varepsilon\rangle_{n} \approx 1 / \sqrt{\mathcal{N}_{n}}$ où $\mathcal{N}_{n}$ est le nombre de trajectoires ayant subi $n$ sauts pendant $t$; ceci conduit à des franges non physiques à valeurs négatives dans la distribution de Wigner près de l'axe $O x_{\alpha}$. Pour minimiser cet effet et le rendre imperceptible à une résolution en $p_{\alpha}$ pas trop haute $\left(\mathrm{d} p_{\alpha}=0,044\right.$ sur la figure $\left.5 \mathrm{~b}\right)$, on arrête la simulation Monte-Carlo à un stade où il y a exactement le même nombre $\mathcal{N}_{n} / 2$ de fonctions d'onde paires et impaires. Pour obtenir une superposition cohérente d'états comprimés, il faudrait effectuer une post-sélection supplémentaire, en se limitant aux réalisations Monte-Carlo de fonction d'onde $\psi\left(p_{\alpha}, t\right)$ de parité fixée $\varepsilon$ (ayant subi un nombre pair de sauts simples si $\varepsilon=1$, un nombre impair sinon, l'opérateur de saut $C_{s}$ changeant la parité). Dans l'opérateur densité conditionnel

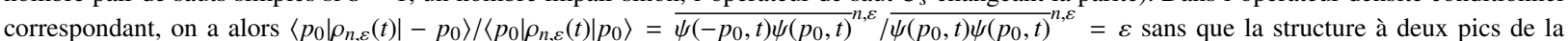
distribution de $P_{\alpha}$ ne soit affectée aux temps longs car $\Pi_{t}\left(p_{\alpha} \mid n, \varepsilon\right) / \Pi_{t}\left(p_{\alpha} \mid n\right) \propto 1+\varepsilon\left[\left(\Gamma_{0}-\Gamma_{\text {ex }} p_{\alpha}^{2}\right) /\left(\Gamma_{0}+\Gamma_{\text {ex }} p_{\alpha}^{2}\right)\right]^{n} \rightarrow 1$ lorsque $n \rightarrow+\infty$ à $p_{\alpha}$ non nul fixé. La distribution de Wigner $W_{t}\left(x_{\alpha}, p_{\alpha} \mid n, \varepsilon\right)$ présente désormais des franges positives et négatives d'amplitude maximale 2 sur l'axe $p_{\alpha}=0$. Cette technique de filtrage vient à bout aussi des mécanismes de décohérence de la section 4.2.4 car les fonctions d'onde Monte-Carlo restent de parité bien définie après action de l'opérateur de saut correspondant $\gamma_{\alpha}^{1 / 2} \alpha$. Cette idée de contrôler la décohérence au travers de mesures de parité est bien connue en électrodynamique quantique en cavité [31]. 

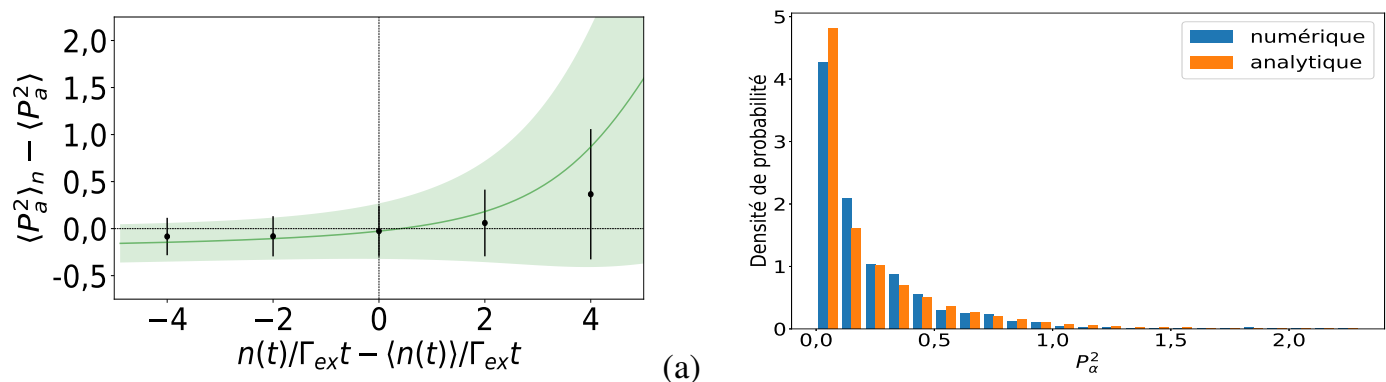

(b)

Figure 4 - Compression de $P_{a}^{2}$ par comptage de photons aux temps courts, $\Gamma_{\mathrm{ex}} t=15$, où $\Gamma_{\mathrm{ex}}$ est le taux (32) de création d'excitations dans le mode bosonique nucléaire hybridé $\alpha$. (a) Moyenne et écart-type conditionnels de la quadrature du spin nucléaire au carré $P_{a}^{2}$ sachant que $n$ photodétections ont eu lieu dans l'intervalle de temps [0, $t$, en fonction de ce nombre $n$. L'écart-type est représenté sous la forme d'un intervalle de confiance. La moyenne inconditionnelle $\left\langle P_{a}^{2}\right\rangle=1 / 4$ est indépendante du temps, voir l'équation (21). Points noirs et barres d'erreur : simulation numérique du modèle à 3 modes avec 3000 réalisations; ligne verte et zone colorée : prédictions analytiques tirées des équations (44), (47) et (50) du modèle à un mode. En pratique, les points noirs sont obtenus après moyenne sur des classes de valeurs de $n$ centrées sur ces points (dans une classe donnée, les trajectoires ont des nombres de photodétections proches mais des histoires indépendantes pour les sauts d'échange de métastabilité auxquelles l'expérimentateur n'a pas accès). Paramètres du modèle à 3 modes : $\Omega / \kappa=1 / 3, \gamma_{m} / \kappa=1 / 10, \gamma_{f} / \kappa=1 / 1000$ (si bien que $\Gamma_{\mathrm{ex}} / \kappa=1 / 909$ ), $n_{a}^{\max }=64, n_{b}^{\max }=n_{c}^{\max }=8$. Ceci correspond à $\Gamma_{0} / \Gamma_{\mathrm{ex}}=12500 / 601 \simeq 20,8$ et $\Gamma_{\mathrm{sq}} / \Gamma_{\mathrm{ex}}=601 / 101202 \simeq 1 / 168$ où $\Gamma_{\mathrm{sq}}$ est le taux de compression (49). (b) Pour la classe centrée sur $n=\langle n(t)\rangle$, histogramme des valeurs conditionnelles de $P_{\alpha}^{2}$. Barres bleues : simulation numérique du modèle à trois modes; barres orange : prédictions analytiques tirées de l'équation (46) du modèle à un mode.

photodétection en continu des photons polarisés selon $O y$ sortant de la cavité rend de plus en plus certaine la valeur de $P_{\alpha}^{2}$, et donc dans une large mesure de $I_{z}^{2}$, le carré de la composante selon $O z$ du spin nucléaire collectif, comme on le voit en reliant dans la limite $\Omega \rightarrow 0$ les moments conditionnels de $P_{a}^{2}$, c'est-à-dire de $I_{z}^{2}$, à ceux de $P_{\alpha}^{2}$ :

$$
\left\langle P_{a}^{2}\right\rangle_{n}=\frac{\gamma_{m}}{\gamma_{f}+\gamma_{m}}\left\langle P_{\alpha}^{2}\right\rangle_{n}+\frac{\gamma_{f} / 4}{\gamma_{f}+\gamma_{m}} \quad ; \quad \operatorname{Var}_{n}\left(P_{a}^{2}\right)=\frac{\gamma_{m}^{2}}{\left(\gamma_{f}+\gamma_{m}\right)^{2}} \operatorname{Var}_{n}\left(P_{\alpha}^{2}\right)+\frac{\gamma_{f} \gamma_{m}}{\left(\gamma_{f}+\gamma_{m}\right)^{2}}\left\langle P_{\alpha}^{2}\right\rangle_{n}+\frac{\gamma_{f}^{2} / 8}{\left(\gamma_{f}+\gamma_{m}\right)^{2}}
$$

Puisque la compression porte sur $P_{a}^{2}$ plutôt que sur $P_{a}$, la distribution angulaire conditionnelle du spin nucléaire collectif est bimodale (elle présente comme celle de $P_{\alpha}$ deux pics bien séparés pourvu que $\left.\gamma_{f} / \gamma_{m} \ll\left(2 p_{0}\right)^{2}\right)$; ces structures plus étroites que la limite quantique standard permettent quand même un pointage angulaire plus précis qu'avec un état non comprimé. Nous redéfinissons donc le gain métrologique (13) en remplaçant au troisième membre de cette équation la variance conditionnelle de $P_{a}$ par le carré de la mi-largeur $\delta P_{a}$ des pics centrés en $\pm P_{a, 0}$ de la distribution de probabilité conditionnelle de $P_{a}$, puis en assimilant le centre $P_{a, 0}^{2}$ et la largeur $\delta\left(P_{a}^{2}\right)$ de la distribution de $P_{a}^{2}$ à la moyenne et à l'écart-type conditionnels de $P_{a}^{2}$ :

$$
\xi^{2}=\frac{4\left(\delta P_{a}\right)^{2}}{\eta}=\frac{\left(2 P_{a, 0} \delta P_{a}\right)^{2}}{\eta P_{a, 0}^{2}}=\frac{\left[\delta\left(P_{a}^{2}\right)\right]^{2}}{\eta P_{a, 0}^{2}}=\frac{\operatorname{Var}_{n}\left(P_{a}^{2}\right)}{\eta\left\langle P_{a}^{2}\right\rangle_{n}}
$$

Remarquons que cette expression ne se déduit pas de la méthode des moments exposée dans la section II.B.6 de la référence [4] en prenant $P_{a}^{2}$ comme estimateur, car l'effet de la transformation unitaire $\exp \left(2 \mathrm{i} \theta X_{a}\right)$ (en pratique, une précession du spin nucléaire autour d'un champ magnétique selon $O y$ ) n'est pas de déplacer le pic dans la distribution de $P_{a}^{2}$ mais de le cliver en deux pics centrés en $\left(P_{a, 0} \pm \theta\right)^{2}$.

Effectuons pour terminer une vérification numérique de ces prédictions analytiques dans le modèle à trois modes. Sur la figure 4a, nous représentons la moyenne conditionnelle du carré $P_{a}^{2}$ de la quadrature de spin nucléaire sachant que $n$ photodétections se sont produites dans l'intervalle de temps $[0, t]$, avec $\Gamma_{\mathrm{ex}} t=15$ (points noirs), en fonction de ce nombre $n$. L'ensemble des réalisations est divisé en 5 classes correspondant à un nombre de photodétections 


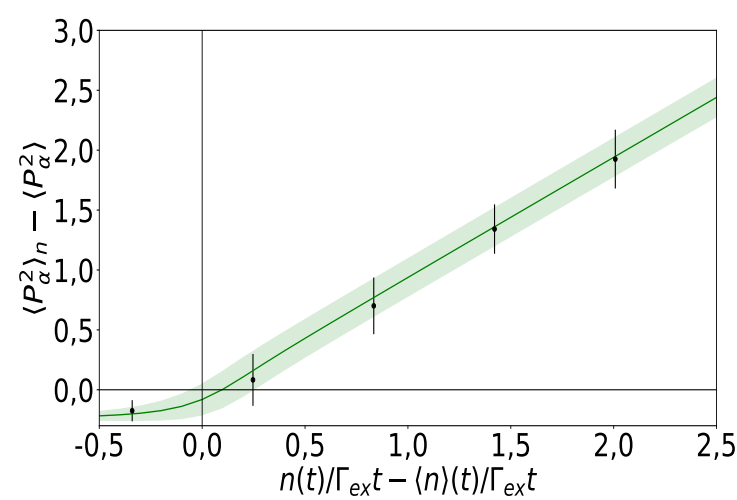

(a)

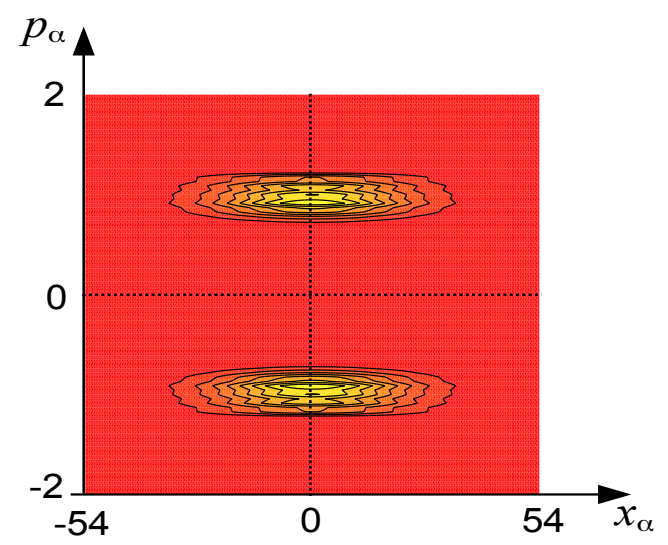

(b)

Figure 5 - Compression de $P_{\alpha}^{2}$ par comptage de photons aux temps longs dans le modèle à un mode (37). (a) Moyenne et écart-type conditionnels de $P_{\alpha}^{2}$ sachant que le nombre de photodétections $n$ tombe dans une classe de valeurs donnée, de manière similaire à la figure 4a mais pour $\Gamma_{\mathrm{ex}} t=1000$ et 2000 réalisations (ce temps long rendrait difficile une simulation dans le modèle à trois modes). (b) Distribution de Wigner du mode bosonique nucléaire hybridé dans l'espace des quadratures $\left(X_{\alpha}, P_{\alpha}\right)$ à $\Gamma_{\mathrm{ex}} t=1000$, obtenue par moyennage des dyades $|\psi(t)\rangle\langle\psi(t)|$ dont le nombre de photodétections tombe dans la 3ème classe de (a) (302 trajectoires sur 5000 réalisations). Elle présente deux lignes de crête mais pas de franges d'interférence (voir la note 14).

tombant dans un intervalle donné, et les points noirs sont obtenus en moyennant sur les réalisations dans une même classe. Les résultats numériques sont proches des prédictions analytiques tirées de (47) et (50) et représentées en vert, sauf dans les classes extrêmes qui comportent un nombre trop faible de réalisations. En revanche, les prédictions analytiques asymptotiques (48), non représentées, seraient en désaccord avec les simulations des deux modèles car le temps $t=15 / \Gamma_{\mathrm{ex}}$ n'est pas assez long, il est très inférieur au temps de compression $1 / \Gamma_{\mathrm{sq}}$. Sur la figure $4 \mathrm{~b}$, nous représentons la distribution de probabilité conditionnelle de $P_{\alpha}^{2}$ correspondant à la classe centrale de la figure $4 \mathrm{a}$; il y a là aussi bon accord entre analytique à un mode et numérique à trois modes. Sur la figure 5 , nous explorons justement les temps longs dans le modèle à un mode, avec $\Gamma_{\mathrm{ex}} t=1000$ c'est-à-dire $\Gamma_{\mathrm{sq}} t \simeq 5,94$. La figure 5a, qui est l'équivalent de la figure $4 \mathrm{a}$, montre que $\left\langle P_{\alpha}^{2}\right\rangle_{n}$ est alors relié au nombre de photodétections $n$ comme dans la prédiction analytique (48), c'est-à-dire selon la première bissectrice dans les unités de la figure, avec un écart-type conditionnel dans (48) à peu près constant $\simeq\left(\Gamma_{0} t\right)^{1 / 2} / \Gamma_{\mathrm{ex}} t \simeq 1 /\left(\Gamma_{\mathrm{sq}} t\right)^{1 / 2}$ car $\Gamma_{0}$ est ici $\gg \Gamma_{\mathrm{ex}}$.

\section{2. Étude de la compression par détection homodyne : solution analytique à un mode et à trois modes}

Nous supposons maintenant que les photons sortant de la cavité polarisés selon $O y$ sont mesurés en continu par détection homodyne [32], comme sur la figure 1c. Il nous faut d'abord trouver les bonnes équations stochastiques donnant l'évolution du vecteur d'état du système conditionnée à la détection homodyne, puisque les opérateurs de saut apparaissant naturellement dans l'écriture (25) ou (37) de l'équation pilote à trois modes ou à un mode sont inadaptés. Nous présentons ensuite quelques résultats analytiques obtenus dans le modèle à un mode puis dans le modèle à trois modes, avant de discuter brièvement l'effet du temps de cohérence fini des atomes métastables. Afin d'obtenir ces résultats, nous avons utilisé le fait que, pour l'état initial vide considéré ici, le vecteur d'état conditionnel est donné exactement à tout temps par un ansatz gaussien [33], quel que soit le nombre de modes du modèle, en présence ou en absence de décohérence. 


\subsubsection{Formulation stochastique adaptée de l'équation pilote}

Une équation pilote générale de la forme de Lindblad [34]

$$
\frac{\mathrm{d} \rho}{\mathrm{d} t}=\frac{1}{\mathrm{i} \hbar}[H, \rho]+\sum_{m} C_{m} \rho C_{m}^{\dagger}-\frac{1}{2}\left\{C_{m}^{\dagger} C_{m}, \rho\right\}
$$

avec $H$ la partie hermitienne de l'hamiltonien et $C_{m}$ les opérateurs de saut, peut être réécrite de manière équivalente en ajoutant une constante arbitraire aux opérateurs de saut et/ou en les mélangeant par combinaison linéaire unitaire quelconque. Afin de tenir compte d'une détection homodyne sur le champ sortant, on forme, à partir d'un opérateur de saut $C_{m}$ correspondant à une photodétection, les deux opérateurs de saut «homodynes» $D_{m, \pm}[28]$

$$
D_{m,+}=\frac{\mu \mathbb{1}+C_{m}}{\sqrt{2}} \quad ; \quad D_{m,-}=\frac{\mu \mathbb{1}-C_{m}}{\sqrt{2}}
$$

où $\mu^{2}$ a les dimensions d'une pulsation. La mesure de la différence des taux de saut $D_{+}^{\dagger} D_{+}-D_{-}^{\dagger} D_{-}$donne alors accès à une quadrature de $C_{m}$. Ainsi, pour $\mu$ réel et $C_{m}$ correspondant à l'opérateur saut de cavité $C_{c}$, voir l'équation (28), la différence entre les nombres de photons $N_{ \pm}$détectés pendant le court intervalle de temps $\Delta t$ dans les deux voies de sortie de la figure 1c, qui constitue par définition le signal homodyne,

$$
N_{+}=\left(D_{c,+}^{\dagger} D_{c,+}\right) \Delta t \quad ; \quad N_{-}=\left(D_{c,-}^{\dagger} D_{c,-}\right) \Delta t \quad ; \quad \frac{N_{+}-N_{-}}{2 \mu}=\frac{c+c^{\dagger}}{2} \sqrt{\kappa} \Delta t
$$

donne accès à $X_{c}$; c'est bien la quadrature du champ conjuguée à $P_{c}$ donc translatée d'une quantité proportionnelle à $P_{b}$ et au temps sous l'action de l'hamiltonien $H$ (12), ce qui renseigne sur $P_{a}$ au travers des collisions d'échange de métastabilité. Dans le cas de l'équation pilote à 3 modes (25), on est obligé d'appliquer la procédure de dédoublement (53) a priori seulement à l'opérateur de saut de cavité. En pratique, nous l'appliquerons également à l'opérateur de saut $C_{\beta}$, c'est-à-dire que nous dédoublerons par homodynage tous les opérateurs de saut $C_{m}$, afin d'éviter l'inconfort d'une représentation mixte mêlant sauts quantiques discrets et évolution stochastique continue, voir l'équation (55) à venir. Dans le cas de l'équation pilote à un mode (37), il faut de toute façon «homodyner » les deux opérateurs de saut $C_{s}$ et $C_{d}$, puisque chacun s'accompagne de la perte d'un photon en cavité, comme l'explique la section 3.4.

Dans la limite d'un oscillateur local de grande amplitude $\mu$, on peut faire comme si $\Delta t$ était infinitésimal ${ }^{15}$ et représenter l'évolution de la fonction d'onde Monte-Carlo, cette fois normalisée à l'unité, par une équation stochastique non linéaire continue sans sauts quantiques $[28,35,36]$ en point de vue d'Ito :

$$
\begin{aligned}
\mathrm{d}|\phi(t)\rangle & =-\frac{\mathrm{i}}{\hbar} H|\phi(t)\rangle \mathrm{d} t-\frac{1}{2} \sum_{m}\left(C_{m}^{\dagger} C_{m}-\left\langle\phi(t)\left|C_{m}+C_{m}^{\dagger}\right| \phi(t)\right\rangle C_{m}+\frac{1}{4}\left\langle\phi(t)\left|C_{m}+C_{m}^{\dagger}\right| \phi(t)\right\rangle^{2}\right)|\phi(t)\rangle \mathrm{d} t \\
& +\sum_{m}\left(C_{m}-\frac{1}{2}\left\langle\phi(t)\left|C_{m}+C_{m}^{\dagger}\right| \phi(t)\right\rangle\right)|\phi(t)\rangle \mathrm{d} \zeta_{m}(t)
\end{aligned}
$$

où, à chaque opérateur de saut $C_{m}$ dans l'écriture initiale de l'équation pilote, on associe un processus stochastique en temps continu $\mathrm{d} \zeta_{m}(t)$, à valeurs réelles, gaussien, de moyenne nulle, de variance $\mathrm{d} t$, statistiquement indépendant des autres processus et sans mémoire. Au même niveau d'approximation, l'opérateur de signal homodyne (54) est remplacé par la somme de sa moyenne et d'un bruit classique représentant ses fluctuations, qui n'est autre que le d $\zeta_{m}$ correspondant [28] :

$$
\frac{N_{+}-N_{-}}{2 \mu}=\frac{\sqrt{\kappa}\left\langle\phi\left|c+c^{\dagger}\right| \phi\right\rangle}{2} \mathrm{~d} t+\frac{1}{2} \mathrm{~d} \zeta_{c}
$$

En pratique, plus que l'historique d'homodynage, c'est-à-dire la dépendance en temps détaillée du signal de détection homodyne, c'est sa moyenne temporelle sur un intervalle de temps $[0, t]$ qui est facilement accessible dans une expérience et permet une post-sélection des états (c'est-à-dire des réalisations expérimentales) sur un ensemble de poids

15. Cette approximation est valable pour une résolution en temps, c'est-à-dire un pas temporel $\Delta t$, telle que $\mu^{-2} \ll \Delta t \ll \kappa^{-1}$, où $\kappa$ est en pratique le taux d'évolution le plus rapide du système dans l'expérience. 

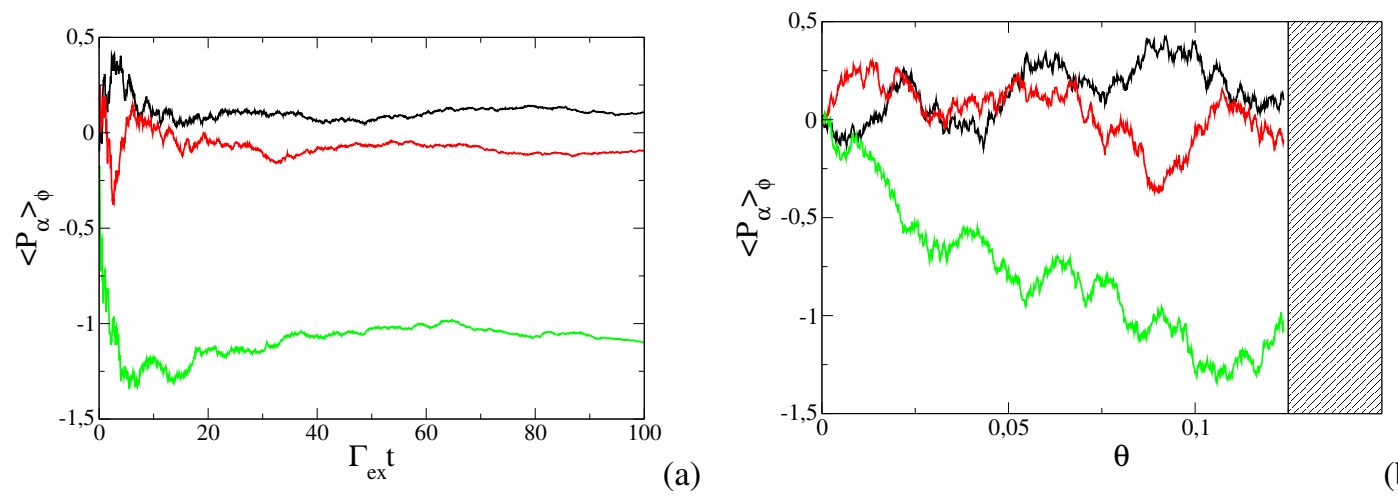

(a)

(b)

FIGURE 6 - Dans le cas de la compression par détection homodyne en continu, marche au hasard (61) effectuée dans le modèle à un mode par la valeur moyenne quantique de la quadrature $P_{\alpha}$ du spin nucléaire dans une réalisation donnée de l'expérience. (a) Valeur moyenne en fonction du temps vrai $t$ adimensionné par le taux (32) de création d'excitations pour trois réalisations de l'expérience; il s'agit d'un mouvement brownien étiré convergeant aux temps longs vers une valeur fixe mais imprédictible. (b) Idem en fonction du temps renormalisé compact $\theta$ (62); il s'agit cette fois d'un mouvement brownien ordinaire mais limité à $\theta \leq 1 / 8$.

statistique non négligeable. Nous introduisons donc le signal intégré ayant la dimension de la racine d'une pulsation,

$$
\sigma(t) \equiv \frac{N_{+}^{\mathrm{tot}}-N_{-}^{\mathrm{tot}}}{2 \mu t}=\frac{1}{t} \int_{0}^{t} \mathrm{~d} t^{\prime}\left[\sqrt{\kappa}\left\langle\phi\left(t^{\prime}\right)\left|X_{c}\right| \phi\left(t^{\prime}\right)\right\rangle+\frac{1}{2} \frac{\mathrm{d} \zeta_{c}\left(t^{\prime}\right)}{\mathrm{d} t^{\prime}}\right]
$$

et nous calculerons dans la suite la moyenne et la variance de la quadrature $P_{a}$ du spin nucléaire conditionnées à $\sigma .{ }^{16}$

\subsubsection{Résultats analytiques dans le modèle à un mode}

Écrivons explicitement l'équation stochastique (55) pour le modèle à un mode (37) :

$$
\mathrm{d}|\phi(t)\rangle=-\frac{\mathrm{d} t}{2} \Gamma_{\mathrm{ex}}\left[P_{\alpha}-\bar{P}_{\alpha}(t)\right]^{2}|\phi(t)\rangle+\sqrt{\Gamma_{\mathrm{ex}}} \mathrm{d} \zeta_{s}(t)\left[P_{\alpha}-\bar{P}_{\alpha}(t)\right]|\phi(t)\rangle
$$

avec $\bar{P}_{\alpha}(t) \equiv\left\langle\phi(t)\left|P_{\alpha}\right| \phi(t)\right\rangle$. Le fait marquant est que les sauts associés à l'opérateur $C_{d}$ proportionnel à l'identité, qui ajoutaient du bruit dans la détection par comptage de photons de la section 4.1, ne donnent pas de contribution, l'opérateur $C_{d}$ disparaissant de l'équation d'évolution conditionnelle dans le cas homodyne. En effet, les photons émis lors de ces sauts proviennent de la composante $|1\rangle|1\rangle$ du vecteur d'état (29) contenant une excitation $\beta$, ce qui les rend optiquement incohérents avec le champ lumineux injecté en cavité, c'est-à-dire avec la composante $|0\rangle|0\rangle$ de (29), au sens où $|1\rangle|1\rangle$ contribue à $\left\langle c^{\dagger} c\right\rangle$ mais pas à $\left\langle c+c^{\dagger}\right\rangle$. Il ne reste donc que le processus stochastique $\mathrm{d} \zeta_{s}$ associé à l'opérateur de saut $C_{s}$. Ce processus se confond avec celui $\mathrm{d} \zeta_{c}$ apparaissant dans le signal de détection homodyne (56), $\mathrm{d} \zeta_{s} \equiv \mathrm{d} \zeta_{c}$, fait admis ici mais qui sera établi en revenant au modèle à trois modes dans la section 4.2.3.

L'équation stochastique (58) présente un terme de bruit linéaire et un terme déterministe quadratique en l'opérateur $P_{\alpha}$, réels dans l'espace de Fourier. Pour l'état initial considéré ici, elle est donc résolue exactement par un

16. Rappelons que la moyenne et la variance d'une observable (ici $P_{a}$ ) dans une seule réalisation $|\phi(t)\rangle$ de l'équation stochastique, donc de l'expérience, n'ont en général pas de sens physique, car il n'y a pas de mesure possible de la valeur moyenne d'une observable dans une seule réalisation, il faut au contraire moyenner sur un grand nombre de réalisations de l'expérience ayant évolué pendant $t$ à partir du même cas pur ou opérateur densité initial. Un contre-exemple correspond au cas où l'on peut, par des mesures en continu, remonter à la dépendance en temps de tous les processus stochastiques $\mathrm{d} \zeta_{m}$; c'est le cas du modèle à un mode soumis à une détection homodyne, la dépendance en temps du signal (56) fixant celle de l'unique processus stochastique $\mathrm{d} \zeta_{c}$. Ceci permettrait alors, en principe, de sélectionner, parmi un grand nombre de réalisations de l'expérience, celles conduisant à l'état $|\phi(t)\rangle$ choisi, et d'en déduire les moyennes d'observables dans $|\phi(t)\rangle$; en pratique, ce serait irréaliste, compte tenu du poids statistique infinitésimal des réalisations à garder. 
ansatz gaussien sur la fonction d'onde en représentation impulsion, réel et correctement normalisé pour la relation de commutation $\left[X_{\alpha}, P_{\alpha}\right]=\mathrm{i} / 2$ :

$$
\left\langle p_{\alpha} \mid \phi(t)\right\rangle=[2 \pi u(t)]^{1 / 4} \exp \left\{-u(t)\left[p_{\alpha}-\bar{P}_{\alpha}(t)\right]^{2}\right\}
$$

En revanche, la gaussianité est perdue dans la compression par photodétection de la section 4.1. En utilisant le calcul d'Ito, ${ }^{17}$ on trouve que $u$ suit une équation d'évolution déterministe, à intégrer avec la condition initiale $u(0)=1$ :

$$
\mathrm{d} u(t)=\Gamma_{\text {ex }} \mathrm{d} t \quad \text { donc } \quad u(t)=1+\Gamma_{\text {ex }} t \quad \text { et } \quad \operatorname{Var}_{\phi} P_{\alpha}(t) \equiv \frac{1}{4 u(t)}=\frac{1}{4} \frac{1}{1+\Gamma_{\text {ex }} t}
$$

où nous avons donné aussi la variance de $P_{\alpha}$ dans l'état $|\phi\rangle$. En revanche, l'équation sur la valeur moyenne de $P_{\alpha}$ dans $|\phi\rangle$ est purement stochastique, avec un coefficient de diffusion $D(t)$ dépendant du temps et la condition initale $\bar{P}_{\alpha}(0)=0$ :

$$
\mathrm{d} \bar{P}_{\alpha}(t)=[2 D(t)]^{1 / 2} \mathrm{~d} \zeta_{s}(t) \quad \text { avec } \quad D(t)=\frac{\Gamma_{\mathrm{ex}}}{8 u(t)^{2}}=\frac{\Gamma_{\mathrm{ex}}}{8\left(1+\Gamma_{\mathrm{ex}} t\right)^{2}}
$$

Comme $D(t)$ est d'intégrale finie, $\bar{P}_{\alpha}(t)$ se stabilise asymptotiquement (aux temps longs) à une valeur fixe sur une seule réalisation, comme on le voit sur la figure 6, avec une variance dans l'état quantique $\operatorname{Var}_{\phi} P_{\alpha}$ tendant vers 0 . Ce phénomène de « convergence stochastique » vers un état propre de l'observable mesurée (en l'occurrence $P_{\alpha}$ ) est attendu dans la description d'une mesure quantique par une équation de diffusion du vecteur d'état [35, 36, 37]. Pour le montrer ici, on introduit un temps $\theta$ renormalisé en termes duquel $\bar{P}_{\alpha}$ effectue un mouvement brownien ordinaire avec un coefficient de diffusion unité, et on remarque que ce temps est borné :

$$
\theta=\int_{0}^{t} \mathrm{~d} t^{\prime} D\left(t^{\prime}\right)=\frac{\Gamma_{\mathrm{ex}} t}{8\left(1+\Gamma_{\mathrm{ex}} t\right)} \underset{t \rightarrow+\infty}{\rightarrow} \theta_{\infty}=\frac{1}{8}
$$

À l'instant renormalisé $\theta_{\infty}, \bar{P}_{\alpha}$ suit une loi gaussienne de moyenne nulle et de variance $1 / 4: \bar{P}_{\alpha}$ a donc la même distribution de probabilité asymptotique $(t \rightarrow+\infty)$ que celle de l'observable $P_{\alpha}$ dans l'état quantique initial du spin nucléaire.

Venons-en maintenant à la moyenne et à la variance de $P_{\alpha}$ conditionnées à la valeur $\mathcal{S}$ du signal d'homodynage intégré en temps $\sigma$ (57). De façon remarquable, nous trouvons que la moyenne conditionnelle est toujours proportionnelle au signal, avec un coefficient de proportionnalité dépendant du temps, et que la variance conditionnelle dépend du temps mais pas du signal :

$$
\left\langle P_{\alpha}\right\rangle_{\sigma=\mathcal{S}}=m(t) \frac{\mathcal{S}}{\sqrt{\Gamma_{\mathrm{ex}}}} \quad \text { où } \quad m(t)=\frac{\Gamma_{\mathrm{ex}} t}{1+\Gamma_{\mathrm{ex}} t} \quad ; \quad \operatorname{Var}_{\sigma=\mathcal{S}}\left(P_{\alpha}\right)=\mathcal{V}(t) \quad \text { où } \quad \mathcal{V}(t)=\frac{1}{4\left(1+\Gamma_{\mathrm{ex}} t\right)}
$$

Ces expressions désignent $\Gamma_{\mathrm{ex}}$ comme le taux de compression du spin nucléaire par homodynage dans le modèle à un mode donc à faible couplage de Faraday :

$$
\Gamma_{\mathrm{sq}} \underset{\Omega \rightarrow 0}{\sim} \Gamma_{\mathrm{ex}}=\frac{\Omega^{2} \gamma_{f}}{\kappa\left(\gamma_{m}+\gamma_{f}\right)}
$$

Sur la figure 7a, nous représentons $m(t)$ et $\mathcal{V}(t)$ en fonction du temps réduit $\Gamma_{\text {ex }} t$. De même que la variance quantique sur une réalisation $\operatorname{Var}_{\phi} P_{\alpha}$, avec laquelle elle coïncide en fait, la variance conditionnelle tend asymptotiquement vers zéro comme l'inverse du temps. Dans la moyenne conditionnelle, le coefficient $m(t)$ tend vers 1 aux temps longs. Pour le comprendre, relions le signal intégré (57) à $\bar{P}_{\alpha}$ en utilisant les expressions adiabatiques (30) dans le vecteur d'état tronqué (29) :

$$
\sigma(t)=\frac{1}{t} \int_{0}^{t} \mathrm{~d} t^{\prime}\left[\sqrt{\Gamma_{\mathrm{ex}}} \bar{P}_{\alpha}\left(t^{\prime}\right)+\frac{1}{2} \frac{\mathrm{d} \zeta_{s}\left(t^{\prime}\right)}{\mathrm{d} t^{\prime}}\right]
$$

17. On ne garde que les termes linéaires en $\mathrm{d} t$ ou en le bruit, et on remplace systématiquement les termes quadratiques $\mathrm{d} \zeta_{s}^{2}$ par leur moyenne $\mathrm{d} t$. 
Comme $\bar{P}_{\alpha}(t)$ se stabilise asymptotiquement sur une seule réalisation, et que la moyenne temporelle du bruit $\mathrm{d} \zeta_{s}$ tend vers zéro comme $1 / t^{1 / 2}$ presque sûrement, $\sigma(+\infty)$ donne directement la valeur de $\bar{P}_{\alpha}(+\infty)$ à un facteur constant $\sqrt{\Gamma_{\mathrm{ex}}}$ près.

Pour établir les résultats (63), relions d'abord la variance conditionnelle de l'opérateur $P_{\alpha}$ à celle de sa moyenne quantique sur une réalisation $\bar{P}_{\alpha}$ comme suit :

$$
\begin{aligned}
\operatorname{Var}_{\sigma=\mathcal{S}}\left(P_{\alpha}\right) & \equiv\left\langle\left\langle\phi\left|P_{\alpha}^{2}\right| \phi\right\rangle\right\rangle_{\sigma=\mathcal{S}}-\left\langle\left\langle\phi\left|P_{\alpha}\right| \phi\right\rangle\right\rangle_{\sigma=\mathcal{S}}^{2}=\left\langle\left\langle\phi\left|P_{\alpha}^{2}\right| \phi\right\rangle-\left\langle\phi\left|P_{\alpha}\right| \phi\right\rangle^{2}\right\rangle_{\sigma=\mathcal{S}}+\left\langle\bar{P}_{\alpha}^{2}\right\rangle_{\sigma=\mathcal{S}}-\left\langle\bar{P}_{\alpha}\right\rangle_{\sigma=\mathcal{S}}^{2} \\
& =\left\langle\operatorname{Var}_{\phi} P_{\alpha}\right\rangle_{\sigma=\mathcal{S}}+\operatorname{Var}_{\sigma=\mathcal{S}}\left(\bar{P}_{\alpha}\right)=\frac{1}{4} \frac{1}{1+\Gamma_{\mathrm{ex}} t}+\operatorname{Var}_{\sigma=\mathcal{S}}\left(\bar{P}_{\alpha}\right)
\end{aligned}
$$

où nous avons utilisé l'expression (60) de la variance quantique de $P_{\alpha}$ dans l'état $|\phi\rangle$. Il reste donc à déterminer la distribution de probabilité conditionnelle de $\bar{P}_{\alpha}$ sachant que $\sigma=\mathcal{S}$,

$$
P\left(\bar{P}_{\alpha}=p_{\alpha} \mid \sigma=\mathcal{S}\right) \equiv \frac{P\left(\bar{P}_{\alpha}=p_{\alpha}, \sigma=\mathcal{S}\right)}{P(\sigma=\mathcal{S})}
$$

Or, la variable aléatoire $\bar{P}_{\alpha}(t)$, résultant d'un mouvement brownien (61), a une distribution de probabilité gaussienne; il en va de même pour l'intégrale temporelle de $\bar{P}_{\alpha}$ et du bruit $\mathrm{d} \zeta_{s}$, donc du signal $\sigma$ (65) qui en est la somme. Comme les variables $\bar{P}_{\alpha}$ et $\sigma$ sont de moyennes nulles, leur distribution de probabilité conjointe est caractérisée par leur matrice de covariance, ou plus directement par sa matrice inverse, si bien que

$$
\begin{aligned}
P\left(\bar{P}_{\alpha}=p_{\alpha} \mid \sigma=\mathcal{S}\right) & =\frac{\frac{1}{2 \pi \sqrt{\left\langle\bar{P}_{\alpha}^{2}\right\rangle_{\text {stoch }}\left\langle\sigma^{2}\right\rangle_{\text {stoch }}-\left\langle\sigma \bar{P}_{\alpha}\right\rangle_{\text {stoch }}^{2}}} \exp \left(-\frac{1}{2} \frac{p_{\alpha}^{2}\left\langle\sigma^{2}\right\rangle_{\text {stoch }}+\mathcal{S}^{2}\left\langle\bar{P}_{\alpha}^{2}\right\rangle_{\text {stoch }}-2 p_{\alpha} \mathcal{S}\left\langle\sigma \bar{P}_{\alpha}\right\rangle_{\text {stoch }}}{\left\langle\bar{P}_{\alpha}^{2}\right\rangle_{\text {stoch }}\left\langle\sigma^{2}\right\rangle_{\text {stoch }}-\left\langle\sigma \bar{P}_{\alpha}\right\rangle_{\text {stoch }}^{2}}\right)}{\frac{1}{\sqrt{2 \pi\left\langle\sigma^{2}\right\rangle_{\text {stoch }}}} \exp \left(-\frac{\mathcal{S}^{2}}{2\left\langle\sigma^{2}\right\rangle_{\text {stoch }}}\right)} \\
& =\frac{1}{\sqrt{2 \pi\left[\left\langle\bar{P}_{\alpha}^{2}\right\rangle_{\text {stoch }}-\left\langle\sigma \bar{P}_{\alpha}\right\rangle_{\text {stoch }}^{2} /\left\langle\sigma^{2}\right\rangle_{\text {stoch }}\right]}} \exp \left[-\frac{1}{2} \frac{\left(p_{\alpha}-\mathcal{S}\left\langle\sigma \bar{P}_{\alpha}\right\rangle_{\text {stoch }} /\left\langle\sigma^{2}\right\rangle_{\text {stoch }}\right)^{2}}{\left\langle\bar{P}_{\alpha}^{2}\right\rangle_{\text {stoch }}-\left\langle\sigma \bar{P}_{\alpha}\right\rangle_{\text {stoch }} /\left\langle\sigma^{2}\right\rangle_{\text {stoch }}}\right]
\end{aligned}
$$

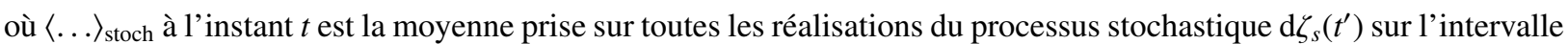
de temps $[0, t]$. On en déduit que, dans les équations (63),

$$
m(t)=\sqrt{\Gamma_{\mathrm{ex}}} \frac{\left\langle\sigma(t) \bar{P}_{\alpha}(t)\right\rangle_{\text {stoch }}}{\left\langle\sigma^{2}(t)\right\rangle_{\text {stoch }}} \text { et } \quad \mathcal{V}(t)=\frac{1}{4\left(1+\Gamma_{\mathrm{ex}} t\right)}+\left\langle\bar{P}_{\alpha}^{2}(t)\right\rangle_{\text {stoch }}-\frac{\left\langle\sigma(t) \bar{P}_{\alpha}(t)\right\rangle_{\text {stoch }}^{2}}{\left\langle\sigma^{2}(t)\right\rangle_{\text {stoch }}}
$$

Afin de déterminer leurs variances et covariance, on écrit $\sigma(t)$ et $\bar{P}_{\alpha}(t)$ comme des fonctionnelles linéaires du processus stochastique $\mathrm{d} \zeta_{s}$ et on utilise le fait que les forces de Langevin $\mathrm{d} \zeta_{s}(t) / \mathrm{d} t$ et $\mathrm{d} \zeta_{s}\left(t^{\prime}\right) / \mathrm{d} t^{\prime}$ ont une fonction de corrélation de Dirac $\delta\left(t-t^{\prime}\right)$. Donnons l'exemple de la première contribution à $\sigma(t)$ :

$$
\int_{0}^{t} \mathrm{~d} t^{\prime \prime} \bar{P}_{\alpha}\left(t^{\prime \prime}\right)=\int_{0}^{t} \mathrm{~d} t^{\prime \prime} \int_{0}^{t^{\prime \prime}} \mathrm{d} t^{\prime}\left[2 D\left(t^{\prime}\right)\right]^{1 / 2} \frac{\mathrm{d} \zeta_{s}\left(t^{\prime}\right)}{\mathrm{d} t^{\prime}}=\int_{0}^{t} \mathrm{~d} t^{\prime} \int_{t^{\prime}}^{t} \mathrm{~d} t^{\prime \prime}\left[2 D\left(t^{\prime}\right)\right]^{1 / 2} \frac{\mathrm{d} \zeta_{s}\left(t^{\prime}\right)}{\mathrm{d} t^{\prime}}=\int_{0}^{t} \mathrm{~d} t^{\prime}\left(t-t^{\prime}\right)\left[2 D\left(t^{\prime}\right)\right]^{1 / 2} \frac{\mathrm{d} \zeta_{s}\left(t^{\prime}\right)}{\mathrm{d} t^{\prime}}
$$

où l'on a changé l'ordre d'intégration sur $t^{\prime}$ et $t^{\prime \prime}$ puis intégré explicitement sur $t^{\prime \prime}$. On aboutit aux expressions cherchées (63), dont la simplicité découle du fait que, sur une réalisation de l'expérience, on a toujours

$$
\sigma(t)=\sqrt{\Gamma_{\mathrm{ex}}} \frac{1+\Gamma_{\mathrm{ex}} t}{\Gamma_{\mathrm{ex}} t} \bar{P}_{\alpha}(t)
$$

De manière remarquable, la connaissance du seul signal intégré $\sigma(t)$ dans une réalisation de l'expérience de durée $t$ suffit à préparer le spin nucléaire dans un cas pur gaussien bien défini (59), avec un paramètre $u$ donné par l'équation (60) et une quadrature moyenne $\bar{P}_{\alpha}$ reliée au signal par l'équation (71).

Pour terminer, revenons à la quadrature $P_{a}$ du spin nucléaire non hybridé, qui est celle véritablement utilisable dans l'expérience une fois la décharge éteinte dans la cellule, comme le montre l'expression (13) du gain métrologique dans 

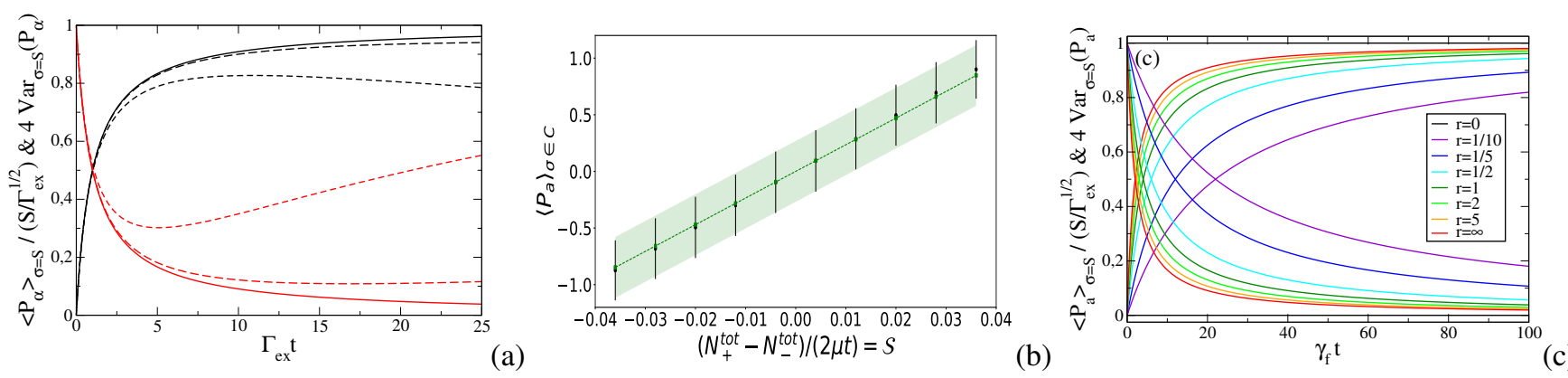

Figure 7 - Compression du spin nucléaire par mesure homodyne en continu. (a) Dans le modèle à un mode, moyenne (en noir) et variance (multipliée par 4, en rouge) de la quadrature $P_{\alpha}$ du spin nucléaire hybridé conditionnées au signal d'homodynage intégré $\sigma$, en fonction du temps d'intégration $t$. Traits pleins : expressions analytiques (63). Tiretés : expressions (102) et (103) en présence de décohérence par désexcitation des métastables sur les parois de la cellule (tireté long : $\epsilon=1 / 100$, tireté court : $\epsilon=1 / 10$, avec $\epsilon=\gamma_{\alpha} / \Gamma_{\text {ex }}$ et $\gamma_{\alpha}$ le taux de décohérence ramené (98)). (b) Dans le modèle à trois modes, pour $\Gamma_{\mathrm{ex}} t=5$, moyenne et écart-type de la quadrature $P_{a}$ du spin nucléaire conditionnées à l'appartenance du signal $\sigma$ à une classe $C$, l'intervalle de valeurs de $\sigma$ ayant été partagé en 10 classes de même largeur (les valeurs $\mathcal{S}$ de $\sigma$ sont en unités de $\Gamma_{\mathrm{ex}}^{1 / 2}$ sur l'axe des abscisses). L'écart-type est représenté sous la forme d'un intervalle de confiance. En noir : simulation numérique de l'équation stochastique (55) avec 1079 réalisations. Tireté vert et zone colorée : résultats exacts tirés des relations (85) et (86), et de l'expression analytique de la distribution de probabilité conditionnelle de $\bar{P}_{a}$ en termes des variances et covariance (92) sur le modèle de l'équation (68). L'écart entre numérique et analytique dans les classes extrêmes est imputable aux faibles nombres de réalisations tombant dans ces classes. Valeurs des paramètres : $\Omega / \kappa=1 / 10, \gamma_{m} / \kappa=1 / 10, \gamma_{f} / \kappa=1 / 100, \Gamma_{\mathrm{ex}} / \kappa=1 / 1000$. (c) Dans la limite $\Gamma_{\mathrm{ex}} \rightarrow 0$ à $\Gamma_{\mathrm{ex}} / 2 \gamma_{f}$ fixé du modèle à trois modes, moyenne et variance conditionnelles de $P_{a}(94)$ en fonction du temps réduit $\gamma_{f} t$, pour différentes valeurs du rapport $r=2 \Gamma_{\mathrm{ex}} / \gamma_{f}$ (courbes croissantes : moyenne, courbes décroissantes : variance).

une mesure de précession. En quittant la base tournée par inversion de la transformation (24) et en limitant l'équation (29) à son premier terme (à l'ordre dominant en $\Omega$ ), il vient

$$
\left\langle P_{a}\right\rangle_{\sigma=\mathcal{S}}=\left(\frac{\gamma_{m}}{\gamma_{f}+\gamma_{m}}\right)^{1 / 2}\left\langle P_{\alpha}\right\rangle_{\sigma=\mathcal{S}} \quad \text { et } \quad \operatorname{Var}_{\sigma=\mathcal{S}}\left(P_{a}\right)=\frac{\gamma_{f}}{4\left(\gamma_{f}+\gamma_{m}\right)}+\frac{\gamma_{m}}{\gamma_{f}+\gamma_{m}} \operatorname{Var}_{\sigma=\mathcal{S}}\left(P_{\alpha}\right)
$$

La variance conditionnelle de $P_{a}$ aux temps longs tend vers une valeur non nulle, bien que faible en pratique : c'est la limite intrinsèque de ce schéma de compression de spin nucléaire, qui utilise l'état métastable de ${ }^{3} \mathrm{He}$ comme intermédiaire.

\subsubsection{Solution du modèle à trois modes}

L'étude de la compression de spin dans le cadre du modèle à un mode est limitée au régime (23) où le taux de compression $\Gamma_{\mathrm{sq}} \sim \Gamma_{\mathrm{ex}}$ correspond à l'échelle de temps la plus longue du système. Il est cependant crucial pour les applications de voir jusqu'où on peut accélérer le processus de compression en augmentant $\Gamma_{\text {ex }}$ donc, par exemple, le couplage de Faraday $\Omega$ des atomes métastables au champ en cavité. À cette fin, nous obtenons la solution analytique du modèle à trois modes en utilisant le caractère gaussien du vecteur d'état qui résulte, comme pour le modèle à un mode, de l'état initial considéré (le vide), de la linéarité des opérateurs de saut $C_{m}$ et de la quadraticité de l'hamiltonien $H$ en les quadratures des modes. L'équation stochastique (55) admet donc comme solution exacte l'ansatz gaussien généralisant celui de l'équation (59),

$$
\left\langle p_{\alpha}, p_{\beta}, x_{c} \mid \phi(t)\right\rangle=\phi(\mathbf{q}, t)=[8 \pi \operatorname{det} \underline{\underline{u}}(t)]^{1 / 4} \exp \{-[\mathbf{q}-\overline{\mathbf{q}}(t)] \cdot \underline{\underline{u}}(t)[\mathbf{q}-\overline{\mathbf{q}}(t)]\} \equiv \mathrm{e}^{-S}
$$

où $\underline{u}$ est une matrice $3 \times 3$ symétrique réelle, $\overline{\mathbf{q}}$ est un vecteur à trois composantes réelles, les coordonnées $q_{\alpha}=p_{\alpha}$ et $q_{\beta} \stackrel{\equiv}{=} p_{\beta}$ sont dans l'espace de Fourier (base propre de la quadrature $P$ ) et la coordonnée $q_{c}=x_{c}$ est dans l'espace des 
«positions » (base propre de la quadrature $X$ ). La seule astuce ici était de choisir comme opérateur de saut d'échange de métastabilité $C_{\beta}=\sqrt{\gamma_{\beta}} \mathrm{i} \beta$; ce choix de phase, qui ne change bien entendu pas l'équation pilote (25), reste légitime pour l'évolution conditionnée à la détection homodyne du champ car les sauts de métastabilité ne sont pas mesurés. Dans la représentation mixte de la fonction d'onde (73), l'hamiltonien $H$ est alors imaginaire pur et les opérateurs de saut sont réels, d'où l'ansatz réel (73). ${ }^{18}$

Pour obtenir les équations du mouvement sur $\underline{\underline{u}}$ et $\overline{\mathbf{q}}$, nous calculons de deux manières différentes la variation relative $\mathrm{d} \phi(\mathbf{q}, t) / \phi(\mathbf{q}, t)$ de la fonction d'onde, d'une part en la reliant à la variation $\mathrm{d} S$ de la quantité $S$ dans (73), séparée en une partie déterministe $\mathrm{d} S_{d}$ et une partie de bruit $\mathrm{d} S_{b}$, d'autre part en reportant l'ansatz (73) dans l'équation stochastique (55). En identifiant les parties déterministes et les parties bruitées des deux formes qui en résultent, nous obtenons

$$
\begin{aligned}
-\mathrm{d} S_{b}= & \gamma_{\beta}^{1 / 2}\left(\frac{1}{2} \partial_{q_{\beta}} S-q_{\beta}+\bar{q}_{\beta}\right) \mathrm{d} \zeta_{\beta}-\kappa^{1 / 2}\left(\frac{1}{2} \partial_{q_{c}} S-q_{c}+\bar{q}_{c}\right) \mathrm{d} \zeta_{c} \\
-\mathrm{d} S_{d}+\frac{1}{2}\left(\mathrm{~d} S_{b}\right)^{2}= & \left(\Omega_{\alpha} q_{\alpha}+\Omega_{\beta} q_{\beta}\right) \frac{\mathrm{d} t}{2} \partial_{q_{c}} S-\frac{\gamma_{\beta} \mathrm{d} t}{2}\left\{q_{\beta}^{2}-\frac{1}{2}+\frac{1}{4}\left[\partial_{q_{\beta}}^{2} S-\left(\partial_{q_{\beta}} S\right)^{2}\right]+\bar{q}_{\beta}\left(\partial_{q_{\beta}} S-2 q_{\beta}\right)+\bar{q}_{\beta}^{2}\right\} \\
& -\frac{\kappa \mathrm{d} t}{2}\left\{q_{c}^{2}-\frac{1}{2}+\frac{1}{4}\left[\partial_{q_{c}}^{2} S-\left(\partial_{q_{c}} S\right)^{2}\right]+\bar{q}_{c}\left(\partial_{q_{c}} S-2 q_{c}\right)+\bar{q}_{c}^{2}\right\}
\end{aligned}
$$

Il reste à reporter dans (75) l'expression de $\mathrm{d} S_{b}$ tirée de (74), en appliquant la règle d'Ito de remplacement des carrés des bruits par leur moyenne, puis à identifier les termes de degré 2 en $\mathbf{q}-\overline{\mathbf{q}}$ pour obtenir l'équation linéaire purement déterministe sur $\underline{\underline{u}}:{ }^{19}$

$$
\begin{array}{lll}
\mathrm{d} u_{\alpha \alpha}=-\Omega_{\alpha} \mathrm{d} t u_{\alpha c} & \mathrm{~d} u_{\alpha \beta}=-\frac{\mathrm{d} t}{2}\left(\gamma_{\beta} u_{\alpha \beta}+\Omega_{\beta} u_{\alpha c}+\Omega_{\alpha} u_{\beta c}\right) & \mathrm{d} u_{\alpha c}=-\frac{\mathrm{d} t}{2}\left(\kappa u_{\alpha c}+\Omega_{\alpha} u_{c c}\right) \\
\mathrm{d} u_{\beta \beta}=-\Omega_{\beta} \mathrm{d} t u_{\beta c}+\gamma_{\beta} \mathrm{d} t\left(1-u_{\beta \beta}\right) & \mathrm{d} u_{\beta c}=-\frac{\mathrm{d} t}{2}\left[\left(\gamma_{\beta}+\kappa\right) u_{\beta c}+\Omega_{\beta} u_{c c}\right] & \mathrm{d} u_{c c}=\kappa \mathrm{d} t\left(1-u_{c c}\right)
\end{array}
$$

et les termes de degré 1 en $\mathbf{q}-\overline{\mathbf{q}}$ pour obtenir l'équation linéaire stochastique sur $\overline{\mathbf{q}}$ :

$$
\mathrm{d} \overline{\mathbf{q}}=\frac{1}{2}\left(\begin{array}{ccc}
0 & 0 & 0 \\
0 & -\gamma_{\beta} & 0 \\
\Omega_{\alpha} & \Omega_{\beta} & -\kappa
\end{array}\right) \mathrm{d} t \overline{\mathbf{q}}+\frac{1}{2}[\mathrm{Id}-\underline{\underline{c}}(t)]\left(\begin{array}{c}
0 \\
\gamma_{\beta}^{1 / 2} \mathrm{~d} \zeta_{\beta}(t) \\
-\kappa^{1 / 2} \mathrm{~d} \zeta_{c}(t)
\end{array}\right)
$$

Faut-il le préciser, $\overline{\mathbf{q}}$ est le vecteur des moyennes quantiques des variables $q$ dans le vecteur d'état $(73) ;{ }^{20}$ par ailleurs, on a introduit la notation $\underline{\underline{c}}$ pour la matrice inverse de $\underline{u}$, qui n'est autre que la matrice de covariance quantique des $q$ à un facteur numérique près. On a donc :

$$
\left\langle\phi(t)\left|q_{i}\right| \phi(t)\right\rangle=\bar{q}_{i}(t) \quad \text { et } \quad\left\langle\phi(t)\left|q_{i} q_{j}\right| \phi(t)\right\rangle=\bar{q}_{i}(t) \bar{q}_{j}(t)+\frac{1}{4} c_{i j}(t) \quad \forall i, j \in\{\alpha, \beta, c\} \quad \text { avec } \quad \underline{\underline{c}}(t)=[\underline{\underline{u}}(t)]^{-1}
$$

18. Par exemple, $\mathrm{i} \beta=\mathrm{i}\left(X_{\beta}+\mathrm{i} P_{\beta}\right)$ est représenté en impulsion par l'opérateur réel $-\partial_{p_{\beta}} / 2-p_{\beta}$, et $\beta^{\dagger} \beta$ par $-\partial_{p_{\beta}}^{2} / 4+p_{\beta}^{2}-1 / 2$.

19. On remarque que les termes quadratiques en $\underline{u}$ au second membre de (75) se compensent avec ceux de $\left(\mathrm{d} S_{b}\right)^{2} / 2$ au premier membre.

20. On peut donc retrouver l'équation (77) à partir de l'équation stochastique déduite de (55) sur la moyenne d'une observable $O$, d $\langle O\rangle=$ $(\mathrm{d} t / \mathrm{i} \hbar)\langle[O, H]\rangle+(\mathrm{d} t / 2) \sum_{m}\left\langle C_{m}^{\dagger}\left[O, C_{m}\right]+\right.$ h.c. $\rangle+\sum_{m}\left[\left\langle O C_{m}+\right.\right.$ h.c. $\left.\rangle-\left\langle C_{m}+C_{m}^{\dagger}\right\rangle\langle O\rangle\right] \mathrm{d} \zeta_{m}$, où $\langle\ldots\rangle$ est prise dans l'état $|\phi(t)\rangle$, en la spécialisant aux cas $O=P_{\alpha}, O=P_{\beta}$ et $O=X_{c}$. 
Le système différentiel (76) s'intègre aisément pour la condition initale $\underline{\underline{u}}(0)=\mathrm{Id}$ :

$$
\begin{aligned}
& u_{\alpha \alpha}(t)=1+\frac{\Omega_{\alpha}^{2} t}{\kappa}-\frac{2 \Omega_{\alpha}^{2}}{\kappa^{2}}\left(1-\mathrm{e}^{-\kappa t / 2}\right) \\
& u_{\alpha \beta}(t)=\frac{\Omega_{\alpha} \Omega_{\beta}}{\gamma_{\beta}}\left(\frac{1}{\gamma_{\beta}+\kappa}+\frac{1}{\kappa}\right)\left(1-\mathrm{e}^{-\gamma_{\beta} t / 2}\right)+\frac{\Omega_{\alpha} \Omega_{\beta}}{\kappa\left(\kappa-\gamma_{\beta}\right)}\left(\mathrm{e}^{-\kappa t / 2}-\mathrm{e}^{-\gamma_{\beta} t / 2}\right)+\frac{\Omega_{\alpha} \Omega_{\beta}}{\kappa\left(\gamma_{\beta}+\kappa\right)}\left(\mathrm{e}^{-\left(\gamma_{\beta}+\kappa\right) t / 2}-\mathrm{e}^{-\gamma_{\beta} t / 2}\right) \\
& u_{\alpha c}(t)=-\frac{\Omega_{\alpha}}{\kappa}\left(1-\mathrm{e}^{-\kappa t / 2}\right) \\
& u_{\beta \beta}(t)=1+\frac{\Omega_{\beta}^{2}}{\gamma_{\beta}\left(\gamma_{\beta}+\kappa\right)}\left(1-\mathrm{e}^{-\gamma_{\beta} t}\right)-\frac{2 \Omega_{\beta}^{2}}{\kappa^{2}-\gamma_{\beta}^{2}}\left(\mathrm{e}^{-\gamma_{\beta} t}-\mathrm{e}^{-\left(\gamma_{\beta}+\kappa\right) t / 2}\right) \\
& u_{\beta c}(t)=-\frac{\Omega_{\beta}}{\gamma_{\beta}+\kappa}\left(1-\mathrm{e}^{-\left(\gamma_{\beta}+\kappa\right) t / 2}\right) \\
& u_{c c}(t)=1
\end{aligned}
$$

Il en serait allé autrement si nous avions pris comme inconnue la matrice de covariance $\underline{\underline{c}}(t)$, qui obéit à un système différentiel non linéaire de Riccati [38]. Comme $\overline{\mathbf{q}}$ décrit un mouvement brownien (partiellement amorti car la matrice de frottement dans (77) est de valeurs propres $0, \gamma_{\beta} / 2$ et $\kappa / 2$ ), et comme le signal homodyne moyenné sur l'intervalle de temps $[0, t] \sigma$ s'en déduit par intégration, ces variables aléatoires ont une statistique gaussienne et nous pouvons reproduire le raisonnement de la section 4.2.2. Nous trouvons pour la moyenne et la variance conditionnelles de la quadrature $P_{a}$ du spin nucléaire sachant que $\sigma=\mathcal{S}$, cette variance déterminant le gain métrologique (13) :

$$
\begin{aligned}
\left\langle P_{a}\right\rangle_{\sigma=\mathcal{S}} & =\frac{\left\langle\sigma(t) \bar{P}_{a}(t)\right\rangle_{\text {stoch }}}{\left\langle\sigma^{2}(t)\right\rangle_{\text {stoch }}} \mathcal{S} \\
\operatorname{Var}_{\sigma=\mathcal{S}}\left(P_{a}\right) & =\frac{1}{4}\left[\frac{\Omega_{\beta}^{2}}{\Omega^{2}} c_{\alpha \alpha}(t)+\frac{\Omega_{\alpha}^{2}}{\Omega^{2}} c_{\beta \beta}(t)-2 \frac{\Omega_{\alpha} \Omega_{\beta}}{\Omega^{2}} c_{\alpha \beta}(t)\right]+\left\langle\bar{P}_{a}^{2}(t)\right\rangle_{\text {stoch }}-\frac{\left\langle\sigma(t) \bar{P}_{a}(t)\right\rangle_{\text {stoch }}^{2}}{\left\langle\sigma^{2}(t)\right\rangle_{\text {stoch }}}=\frac{1}{4}-\frac{\left\langle\sigma(t) \bar{P}_{a}(t)\right\rangle_{\text {stoch }}^{2}}{\left\langle\sigma^{2}(t)\right\rangle_{\text {stoch }}}(86)
\end{aligned}
$$

L'expression entre crochets dans l'équation (86) est l'élément de matrice de $\underline{c}(t)$ dans le vecteur $\left(\Omega_{\beta} / \Omega,-\Omega_{\alpha} / \Omega, 0\right)$

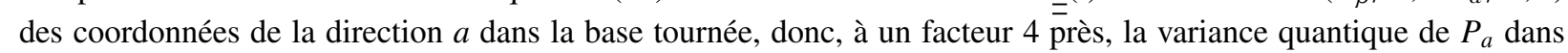
l'état stochastique $\phi(t)$, dépendant du temps mais, rappelons-le, indépendante de la réalisation particulière de $\phi(t)$. L'expression simplifiée au troisième membre découle de la propriété (21) sur la moyenne non conditionnelle $\left\langle P_{a}^{2}\right\rangle(t)=$ $1 / 4$ et de la chaîne d'égalités

$\left\langle P_{a}^{2}\right\rangle(t)=\left\langle\left\langle\phi(t)\left|P_{a}^{2}\right| \phi(t)\right\rangle\right\rangle_{\text {stoch }}=\left\langle\left\langle\phi(t)\left|P_{a}^{2}\right| \phi(t)\right\rangle-\left\langle\phi(t)\left|P_{a}\right| \phi(t)\right\rangle^{2}+\left\langle\phi(t)\left|P_{a}\right| \phi(t)\right\rangle^{2}\right\rangle_{\text {stoch }}=\left\langle\operatorname{Var}_{\phi(t)} P_{a}\right\rangle_{\text {stoch }}+\left\langle\bar{P}_{a}^{2}(t)\right\rangle_{\text {stoch }}$

Il reste, pour déterminer les variance et covariance des variables aléatoires $\bar{P}_{a}(t)$ et $\sigma(t)$, à calculer leurs amplitudes sur les processus stochastiques $\mathrm{d} \zeta_{\beta}\left(t^{\prime}\right)$ et $\mathrm{d} \zeta_{c}\left(t^{\prime}\right)$, en intégrant formellement l'équation (77) par la méthode de variation de la constante pour $\bar{P}_{a}$ et $\bar{X}_{c}$, et en procédant comme dans l'équation (70) pour $\sigma$ :

$$
\begin{aligned}
& p_{\beta}\left(t, t^{\prime}\right)=-\frac{1}{2} \gamma_{\beta}^{1 / 2}\left\{\frac{\Omega_{\beta}}{\Omega} c_{\alpha \beta}\left(t^{\prime}\right)+\frac{\Omega_{\alpha}}{\Omega}\left[1-c_{\beta \beta}\left(t^{\prime}\right)\right] \mathrm{e}^{-\gamma_{\beta}\left(t-t^{\prime}\right) / 2}\right\} \\
& p_{c}\left(t, t^{\prime}\right)=\frac{1}{2} \kappa^{1 / 2}\left\{\frac{\Omega_{\beta}}{\Omega} c_{\alpha c}\left(t^{\prime}\right)-\frac{\Omega_{\alpha}}{\Omega} c_{\beta c}\left(t^{\prime}\right) \mathrm{e}^{-\gamma_{\beta}\left(t-t^{\prime}\right) / 2}\right\} \\
& \sigma_{\beta}\left(t, t^{\prime}\right)=\frac{\left(\gamma_{\beta} \kappa\right)^{1 / 2}}{2 t}\left\{-c_{\alpha \beta}\left(t^{\prime}\right)\left[t-t^{\prime}-f_{\kappa}\left(t-t^{\prime}\right)\right] \frac{\Omega_{\alpha}}{\kappa}+\left[1-c_{\beta \beta}\left(t^{\prime}\right)\right]\left[f_{\gamma_{\beta}}\left(t-t^{\prime}\right)-f_{\kappa}\left(t-t^{\prime}\right)\right] \frac{\Omega_{\beta}}{\kappa-\gamma_{\beta}}-c_{\beta c}\left(t^{\prime}\right) f_{\kappa}\left(t-t^{\prime}\right)\right\} \\
& \sigma_{c}\left(t, t^{\prime}\right)=\frac{1}{2 t}-\frac{\kappa}{2 t}\left\{-c_{\alpha c}\left(t^{\prime}\right)\left[t-t^{\prime}-f_{\kappa}\left(t-t^{\prime}\right)\right] \frac{\Omega_{\alpha}}{\kappa}-c_{\beta c}\left(t^{\prime}\right)\left[f_{\gamma_{\beta}}\left(t-t^{\prime}\right)-f_{\kappa}\left(t-t^{\prime}\right)\right] \frac{\Omega_{\beta}}{\kappa-\gamma_{\beta}}+\left[1-c_{c c}\left(t^{\prime}\right)\right] f_{\kappa}\left(t-t^{\prime}\right)\right\}
\end{aligned}
$$


où $f_{\lambda}(\tau) \equiv[1-\exp (-\lambda \tau / 2)] /(\lambda / 2)$. On obtient :

$$
\begin{aligned}
\left\langle\sigma(t) \bar{P}_{a}(t)\right\rangle_{\text {stoch }} & =\int_{0}^{t} \mathrm{~d} t^{\prime}\left[p_{\beta}\left(t, t^{\prime}\right) \sigma_{\beta}\left(t, t^{\prime}\right)+p_{c}\left(t, t^{\prime}\right) \sigma_{c}\left(t, t^{\prime}\right)\right] \quad ; \quad\left\langle\sigma^{2}(t)\right\rangle_{\text {stoch }}=\int_{0}^{t} \mathrm{~d} t^{\prime}\left[\sigma_{\beta}^{2}\left(t, t^{\prime}\right)+\sigma_{c}^{2}\left(t, t^{\prime}\right)\right] ; \\
\left\langle\bar{P}_{a}^{2}(t)\right\rangle_{\text {stoch }} & =\int_{0}^{t} \mathrm{~d} t^{\prime}\left[p_{\beta}^{2}\left(t, t^{\prime}\right)+p_{c}^{2}\left(t, t^{\prime}\right)\right]
\end{aligned}
$$

On déduit de ces résultats les limites aux temps longs ${ }^{21}$

$$
\left\langle P_{a}\right\rangle_{\sigma=\mathcal{S}} \underset{t \rightarrow+\infty}{\rightarrow}\left(\frac{\gamma_{m}}{\gamma_{f}+\gamma_{m}}\right)^{1 / 2} \frac{\mathcal{S}}{\Gamma_{\mathrm{ex}}^{1 / 2}} \quad ; \quad \operatorname{Var}_{\sigma=\mathcal{S}}\left(P_{a}\right) \underset{t \rightarrow+\infty}{\rightarrow} \frac{1}{4} \frac{\gamma_{f}}{\gamma_{f}+\gamma_{m}}
$$

avec lesquelles les prédictions (72) du modèle à un mode, pourtant obtenues dans la limite de couplage faible (23), sont en accord parfait.

En application de notre solution analytique du modèle à trois modes, faisons tendre le taux $\Gamma_{\mathrm{ex}}$ vers zéro à temps réduit $\tau=\Gamma_{\mathrm{ex}} t$ fixé en maintenant (contrairement au modèle à un mode) le rapport $\Gamma_{\mathrm{ex}} / \gamma_{f}$ à une valeur constante non infinitésimale. La motivation physique est claire : dans les expériences projetées [14], $\gamma_{f}$ et $\Gamma_{\text {ex }}$ sont du même ordre de grandeur mais sont vraiment beaucoup plus petits que $\gamma_{m}$ et $\kappa$ (par des facteurs $\approx 10^{-6}$ et $10^{-9}$ ). Nous trouvons dans cette limite : 22

$$
\left\langle P_{a}\right\rangle_{\sigma=\mathcal{S}} \sim \frac{\Gamma_{\mathrm{sq}} t}{1+\Gamma_{\mathrm{sq}} t} \frac{\mathcal{S}}{\Gamma_{\mathrm{ex}}^{1 / 2}} \quad \text { et } \quad \operatorname{Var}_{\sigma=\mathcal{S}}\left(P_{a}\right) \sim \frac{1}{4} \frac{1}{1+\Gamma_{\mathrm{sq}} t}
$$

où l'on a introduit le taux de compression du spin nucléaire

$$
\Gamma_{\mathrm{sq}} \equiv\left(\frac{1}{\Gamma_{\mathrm{ex}}}+\frac{2}{\gamma_{f}}\right)^{-1}
$$

On retrouve l'adimensionnement naturel du signal par $\Gamma_{\mathrm{ex}}^{1 / 2}$ déjà constaté dans le modèle à un mode et les mêmes formes fonctionnelles en temps, mais on perd toute relation de proportionnalité de type (71) entre signal intégré et moyenne de quadrature sur une réalisation, la variance conditionnelle de $\bar{P}_{a}$ étant désormais $\not \equiv 0 .{ }^{23}$ Nous représentons sur la figure $7 \mathrm{c}$ la dépendance en le temps adimensionné $\gamma_{f} t$ de la moyenne et de la variance conditionnelles (94) pour différentes valeurs du rapport $r=2 \Gamma_{\mathrm{ex}} / \gamma_{f}$. On remarque que le processus de compression est d'autant plus rapide que $r$ est plus grand, et qu'il sature à un comportement limite. C'était prévisible, car $\Gamma_{\mathrm{sq}}$ est une fonction croissante de $r$ de limite finie; à temps fixé, la moyenne conditionnelle (en unités de $\mathcal{S} / \Gamma_{\mathrm{ex}}^{1 / 2}$ ) est donc une fonction croissante et la variance conditionnelle une fonction décroissante de $r$, comme on le voit sur la figure 7c. Plus précisément, dans la limite de couplage faible $\Omega \rightarrow 0$, où $r \rightarrow 0$, le taux de compression est équivalent au taux de création d'excitations $\Gamma_{\mathrm{ex}}$, en accord avec le modèle à un mode, et dans la limite $r \rightarrow+\infty$, il sature à la valeur $\gamma_{f} / 2$. On ne peut donc comprimer plus rapidement qu'au taux $\gamma_{f}$, ce qui n'est pas surprenant : on ne peut espérer réduire les fluctuations du spin nucléaire avant que chaque atome dans l'état fondamental n'ait subi en moyenne au moins une collision d'échange de métastabilité, le taux effectif $\gamma_{f}$ étant en pratique du même ordre de grandeur que le taux individuel $1 / T$ dans l'équation (8) sauf en cas d'extrême polarisation, voir la figure $3 \mathrm{a}$.

\footnotetext{
21. Donnons quelques résultats et considérations intermédiaires. (i) Alors que $c_{\beta \beta}\left(t^{\prime}\right), c_{\beta c}\left(t^{\prime}\right)$ et $c_{c c}\left(t^{\prime}\right)$ ont une limite finie lorsque $t^{\prime} \rightarrow+\infty$ [on aura besoin de $c_{\beta \beta}(+\infty)=(1+\rho)^{-1}, c_{\beta c}(+\infty)=\Omega_{\beta} /\left(\left(\gamma_{\beta}+\kappa\right)(1+\rho)\right)$ avec $\left.\rho=\Omega_{\beta}^{2} \kappa /\left(\gamma_{\beta}\left(\gamma_{\beta}+\kappa\right)^{2}\right)\right], c_{\alpha \alpha}\left(t^{\prime}\right), c_{\alpha \beta}\left(t^{\prime}\right)$ et $c_{\alpha c}\left(t^{\prime}\right)$ tendent vers zéro comme $1 / t^{\prime}$. (ii) Dans une intégrale sur $t^{\prime}$ contenant le facteur exponentiel exp[- $\left.\gamma_{\beta}\left(t-t^{\prime}\right) / 2\right]$ ou son carré, on peut remplacer la fonction qui le multiplie par sa limite en $t^{\prime}=+\infty$. (iii) Pour toute fonction uniformément bornée $w\left(t, t^{\prime}\right)$, on peut montrer pour $v \in\{\beta, c\}$ que $\int_{0}^{t} \mathrm{~d} t^{\prime}\left[\left(t-t^{\prime}\right) c_{\alpha v}\left(t^{\prime}\right)+\right.$ $\left.w\left(t, t^{\prime}\right)\right]^{2} / t^{2} \rightarrow \int_{0}^{+\infty} \mathrm{d} t^{\prime} c_{\alpha v}^{2}\left(t^{\prime}\right)$. (iv) On obtient alors les limites asymptotiques $\left\langle P_{a}^{2}(t)\right\rangle_{\text {stoch }} \rightarrow\left(\Omega_{\beta} / 2 \Omega\right)^{2} I+\left(\Omega_{\alpha} / 2 \Omega\right)^{2} \rho /(1+\rho),\left\langle\sigma^{2}(t)\right\rangle_{\text {stoch }} \rightarrow$ $\left(\Omega_{\alpha}^{2} / 4 \kappa\right) \mathcal{I},\left\langle\sigma(t) \bar{P}_{a}(t)\right\rangle_{\text {stoch }} \rightarrow\left(\Omega_{\alpha} \Omega_{\beta} / 4 \Omega \kappa^{1 / 2}\right) \mathcal{I}$ où $\mathcal{I} \equiv \int_{0}^{+\infty} \mathrm{d} t^{\prime}\left[\gamma_{\beta} c_{\alpha \beta}^{2}\left(t^{\prime}\right)+\kappa c_{\alpha c}^{2}\left(t^{\prime}\right)\right]$. Nous déduisons ainsi (93) de (85) et de la première égalité dans (86), sans avoir besoin de connaître la valeur de $\mathcal{I}$. On tire de la seconde égalité dans (86) le résultat $\mathcal{I}=1$, que l'on peut déduire aussi de l'équation du mouvement $\mathrm{d} c_{\alpha \alpha} / \mathrm{d} t=-\gamma_{\beta} c_{\alpha \beta}^{2}-\kappa c_{\alpha c}^{2}$ intégrée entre $t=0$ et $t=+\infty$.

22. En pratique, il suffit de faire tendre $\Omega_{\alpha}$ vers zéro à $\tau=\Gamma_{\mathrm{ex}} t>0, \Omega_{\beta}, \gamma_{\beta}$ et $\kappa$ fixés. En particulier, ceci fait disparaître tous les transitoires exponentiels dans les équations (79)-(84). Pour simplifier les calculs, il est utile d'introduire la quantité $\rho=\Omega^{2} \kappa /\left[2 \gamma_{m}\left(\kappa+2 \gamma_{m}\right)^{2}\right]$ si bien que $\rho=\left(\Gamma_{\mathrm{ex}} / 2 \gamma_{f}\right)\left(1+2 \gamma_{m} / \kappa\right)^{-2}$ dans la limite $\gamma_{f} \rightarrow 0$.

23. On a en effet $\operatorname{Var}_{\sigma=\mathcal{S}}\left(\bar{P}_{a}\right) \sim \Gamma_{\mathrm{ex}} t /\left[4\left(1+\Gamma_{\mathrm{ex}} t\right)\right]-\Gamma_{\mathrm{sq}} t /\left[4\left(1+\Gamma_{\mathrm{sq}} t\right)\right]$.
} 


\subsubsection{Effet de la décohérence}

Pour être complets, nous tenons compte, dans le schéma de compression homodyne, de la durée de vie finie $\left(2 \gamma_{0}\right)^{-1}$ des atomes métastables, qui se désexcitent lorsqu'ils atteignent les parois de la cellule après un mouvement diffusif dans le gaz. À cette fin, nous ajoutons un opérateur de saut $\sqrt{2 \gamma_{0}} b$ à l'équation pilote à trois modes (20). Comme la partie autre qu'hamiltonienne hermitienne reste quadratique en les quadratures des modes, elle peut être mise sous forme réduite par une rotation appropriée des modes atomiques, comme nous l'avions fait déjà dans la section 3.4 : il faut décomposer le vecteur $(a, b)$ dans la base propre orthonormale de la matrice des taux

$$
\underline{\underline{\Gamma}}=\left(\begin{array}{cc}
2 \gamma_{f} & -2 \sqrt{\gamma_{f} \gamma_{m}} \\
-2 \sqrt{\gamma_{f} \gamma_{m}} & 2\left(\gamma_{0}+\gamma_{m}\right)
\end{array}\right)
$$

avec des coefficients à valeur opérateur $\alpha$ et $\beta$. La direction $\beta$ reste celle de la valeur propre maximale $\gamma_{\beta}$ de $\underline{\underline{\Gamma}}$, et $\alpha$ celle de la valeur propre minimale $\gamma_{\alpha}$, désormais non nulle. Ceci conduit à l'équation pilote

$$
\frac{\mathrm{d} \rho}{\mathrm{d} t}=\frac{1}{\mathrm{i} \hbar}\left[\hbar\left(\Omega_{\alpha} P_{\alpha}+\Omega_{\beta} P_{\beta}\right) P_{c}, \rho\right]+\kappa\left(c \rho c^{\dagger}-\frac{1}{2}\left\{c^{\dagger} c, \rho\right\}\right)+\gamma_{\alpha}\left(\alpha \rho \alpha^{\dagger}-\frac{1}{2}\left\{\alpha^{\dagger} \alpha, \rho\right\}\right)+\gamma_{\beta}\left(\beta \rho \beta^{\dagger}-\frac{1}{2}\left\{\beta^{\dagger} \beta, \rho\right\}\right)
$$

La nouvelle expression des pulsations de Faraday $\Omega_{\alpha}, \Omega_{\beta}$ et des taux $\gamma_{\alpha}, \gamma_{\beta}$ se trouve dans l'Annexe B, qui donne aussi l'expression analytique de la moyenne et de la variance de la quadrature $P_{a}$ du spin nucléaire conditionnées au signal homodyne intégré, en toute généralité. Nous nous restreignons ici à la limite physiquement utile $\gamma_{0} \ll \gamma_{m}$ (on a toujours $\gamma_{f}<\gamma_{m}$ ). À l'ordre le plus bas en $\gamma_{0}$, les coefficients $\Omega_{\alpha}, \Omega_{\beta}$ et $\gamma_{\beta}$ restent inchangés, et l'on a

$$
\gamma_{\alpha} \simeq \frac{2 \gamma_{0} \gamma_{f}}{\gamma_{m}+\gamma_{f}}
$$

ce qui n'est autre que le taux de décohérence ramené dans le spin nucléaire hybridé. De plus, nous nous plaçons dans la limite (23), avec $\gamma_{\alpha}=O\left(\Gamma_{\mathrm{ex}}\right)$, ce qui permet d'évaluer l'effet de la décohérence en utilisant le modèle à un mode, dont l'obtention reste la même que dans la section 3.4. L'équation stochastique (58) est complétée comme suit,

$$
\mathrm{d}|\phi(t)\rangle=-\frac{\Gamma_{\text {ex }} \mathrm{d} t}{2}\left(P_{\alpha}-\bar{P}_{\alpha}\right)^{2}|\phi(t)\rangle+\sqrt{\Gamma_{\text {ex }}} \mathrm{d} \zeta_{s}(t)\left(P_{\alpha}-\bar{P}_{\alpha}\right)|\phi(t)\rangle-\frac{\gamma_{\alpha} \mathrm{d} t}{2}\left(\alpha^{\dagger} \alpha+2 \mathrm{i} \bar{P}_{\alpha} \alpha+\bar{P}_{\alpha}^{2}\right)|\phi(t)\rangle+\sqrt{\gamma_{\alpha}} \mathrm{d} \zeta_{\alpha}(t)\left(\mathrm{i} \alpha+\bar{P}_{\alpha}\right)|\phi(t)\rangle
$$

Nous avons pris soin de choisir $\gamma_{\alpha}^{1 / 2} \mathrm{i} \alpha$ comme opérateur de saut de la décohérence ramenée (la justification est la même que dans la section 4.2.3, les sauts de décohérence n'étant pas mesurés), ce qui permet de résoudre l'équation par le même ansatz gaussien réel (59). Nous trouvons cette fois ${ }^{24}$

$$
\begin{aligned}
\mathrm{d} u & =\left[\Gamma_{\mathrm{ex}}+\gamma_{\alpha}(1-u)\right] \mathrm{d} t \quad \Longrightarrow \quad u(\tau)=1+\frac{1-\exp (-\epsilon \tau)}{\epsilon} \\
\mathrm{d} \bar{P}_{\alpha} & =-\frac{1}{2} \gamma_{\alpha} \bar{P}_{\alpha} \mathrm{d} t+\frac{\sqrt{\Gamma_{\mathrm{ex}}} \mathrm{d} \zeta_{s}+\sqrt{\gamma_{\alpha}}(u-1) \mathrm{d} \zeta_{\alpha}}{2 u}
\end{aligned}
$$

où nous avons posé $\tau=\Gamma_{\mathrm{ex}} t$ et $\epsilon=\gamma_{\alpha} / \Gamma_{\mathrm{ex}}$. Les mêmes arguments de gaussianité que dans la section 4.2.2 conduisent aux mêmes dépendances en le signal $\mathcal{S}$ de la moyenne et de la variance conditionnelles, ${ }^{25}$

$$
\begin{aligned}
\left\langle P_{\alpha}\right\rangle_{\sigma=\mathcal{S}} & =m(\tau) \frac{\mathcal{S}}{\sqrt{\Gamma_{\mathrm{ex}}}} \text { avec } m(\tau)=\sqrt{\Gamma_{\mathrm{ex}}} \frac{\left\langle\sigma(t) \bar{P}_{\alpha}(t)\right\rangle_{\text {stoch }}}{\left\langle\sigma^{2}(t)\right\rangle_{\text {stoch }}} \\
\operatorname{Var}_{\sigma=\mathcal{S}}\left(P_{\alpha}\right) & =\mathcal{V}(\tau) \quad \text { avec } \quad \mathcal{V}(\tau)=\frac{1}{4}-\frac{\left\langle\sigma(t) \bar{P}_{\alpha}(t)\right\rangle_{\text {stoch }}^{2}}{\left\langle\sigma^{2}(t)\right\rangle_{\text {stoch }}}
\end{aligned}
$$

24. Dans le régime $\epsilon \ll 1$, la limite aux temps longs de la variance de $P_{\alpha}$ sur une seule réalisation dépend fortement du choix de phase dans l'opérateur de saut de la décohérence ramenée, ce qui souligne le caractère non physique de cette variance (voir la note 16) : si l'on prend $\gamma_{\alpha}^{1 / 2} \alpha$ comme opérateur de saut, on trouve que $\operatorname{Var}_{\phi} P_{\alpha} \rightarrow \epsilon^{1 / 2} / 4$ [14] au lieu de $\operatorname{Var}_{\phi} P_{\alpha} \rightarrow 1 / 4 \epsilon$ comme dans l'équation (100). Plus généralement, le choix $\gamma_{\alpha}^{1 / 2} \exp (\mathrm{i} \theta) \alpha,-\pi / 2<\theta<\pi / 2$, conduit à l'équation de Riccati sur le paramètre $u$ de l'ansatz gaussien (désormais complexe) d $u=$ $\Gamma_{\operatorname{ex}} \mathrm{d} t+\gamma_{\alpha} \mathrm{d} t\left\{\exp (2 \mathrm{i} \theta) u+[1-\exp (2 \mathrm{i} \theta)] / 2-u^{2}[1+\exp (2 \mathrm{i} \theta)] / 2\right\}$ si bien que $\operatorname{Var}_{\phi} P_{\alpha} \rightarrow(1 / 4) \epsilon^{1 / 2} \sqrt{\cos \theta} \cos (\theta / 2)$ en un temps $\tau \sim 1 / \sqrt{2 \epsilon[1+\exp (2 \mathrm{i} \theta)]}$ la loi de puissance en $\epsilon$ obtenue pour $\theta=0$ est donc la règle, celle obtenue pour $\theta=\pi / 2$ est l'exception.

25. Nous avons simplifié l'expression (103) à l'aide de l'identité $[4 u(\tau)]^{-1}+\left\langle\bar{P}_{\alpha}^{2}\right\rangle_{\text {stoch }}=1 / 4$, qui résulte comme dans l'équation (87) du fait que la moyenne inconditionnelle $\left\langle P_{\alpha}^{2}\right\rangle=1 / 4$, même en présence de décohérence. 
et les variance et covariance prises sur les processus stochastiques $\mathrm{d} \zeta_{s} \equiv \mathrm{d} \zeta_{c}$ et $\mathrm{d} \zeta_{\alpha}$,

$$
\begin{aligned}
\frac{\left\langle\sigma^{2}\right\rangle_{\text {stoch }}}{\Gamma_{\mathrm{ex}}} & =\int_{0}^{\tau} \frac{\mathrm{d} \tau^{\prime}}{\tau^{2}}\left\{\left[\frac{1}{2}+\frac{1-\mathrm{e}^{\epsilon\left(\tau^{\prime}-\tau\right) / 2}}{\epsilon u\left(\tau^{\prime}\right)}\right]^{2}+\frac{\left[u\left(\tau^{\prime}\right)-1\right]^{2}}{u^{2}\left(\tau^{\prime}\right)} \frac{\left[1-\mathrm{e}^{\epsilon\left(\tau^{\prime}-\tau\right) / 2}\right]^{2}}{\epsilon}\right\}=\frac{\epsilon \tau-2\left(1-\mathrm{e}^{-\epsilon \tau / 2}\right)}{\epsilon^{2} \tau^{2}}+\frac{1}{4 \tau} \\
\frac{\left\langle\sigma \bar{P}_{\alpha}\right\rangle_{\text {stoch }}}{\sqrt{\Gamma_{\mathrm{ex}}}} & =\int_{0}^{\tau} \frac{\mathrm{d} \tau^{\prime}}{\tau} \frac{\mathrm{e}^{\epsilon\left(\tau^{\prime}-\tau\right) / 2}}{2 u\left(\tau^{\prime}\right)}\left\{\frac{1}{2}+\frac{1-\mathrm{e}^{\epsilon\left(\tau^{\prime}-\tau\right) / 2}}{\epsilon u\left(\tau^{\prime}\right)}+\frac{\left[u\left(\tau^{\prime}\right)-1\right]^{2}}{u\left(\tau^{\prime}\right)}\left[1-\mathrm{e}^{\epsilon\left(\tau^{\prime}-\tau\right) / 2}\right]\right\}=\frac{1-\mathrm{e}^{-\epsilon \tau / 2}}{2 \epsilon \tau}
\end{aligned}
$$

Ces expressions permettent d'évaluer facilement l'effet de la décohérence sur la compression de spin au travers du gain métrologique (13), voir les tiretés sur la figure 7a. Pour le cas utile en pratique d'une faible décohérence $\epsilon \ll 1$ et d'un temps court devant $1 / \gamma_{\alpha}$, elles peuvent être développées au premier ordre en $\epsilon$ :

$$
m(\tau)=\frac{\tau}{1+\tau}-\epsilon \frac{(\tau+3) \tau^{2}}{12(\tau+1)^{2}}+O\left(\epsilon^{2} \tau^{2}\right) \quad ; \quad \mathcal{V}(\tau)=\frac{1}{4(\tau+1)}+\epsilon \frac{(\tau+3 / 2) \tau^{2}}{12(\tau+1)^{2}}+O\left(\epsilon^{2} \tau^{2}\right)
$$

On en déduit que la compression optimale sur $P_{\alpha}$ est obtenue à un temps $t_{\mathrm{opt}} \sim\left(3 / \Gamma_{\mathrm{ex}} \gamma_{\alpha}\right)^{1 / 2}$ et correspond à une variance conditionnelle $\mathcal{V}_{\mathrm{opt}} \sim\left(\gamma_{\alpha} / 12 \Gamma_{\mathrm{ex}}\right)^{1 / 2}$. Remarquons qu'on introduit souvent, dans les études de compression de spin dans les gaz d'atomes alcalins en cavité, la coopérativité $C$ du système couplé atome-champ, définie comme le carré de la pulsation de couplage divisé par les taux de décroissance des états couplés [39]. En ce sens, la coopérativité du système spin nucléaire hybridé-champ vaut

$$
C \equiv \frac{\Omega_{\alpha}^{2}}{\kappa \gamma_{\alpha}}=\frac{\Gamma_{\mathrm{ex}}}{\gamma_{\alpha}} \simeq \frac{\Omega^{2}}{2 \gamma_{0} \kappa}
$$

si bien que nous retrouvons la loi d'échelle d'exposant $-1 / 2$, habituelle dans les alcalins, reliant la variance de spin optimale à $C$ [39]. Plus généralement, la décohérence a un effet faible sur la compression du spin nucléaire tant qu'on reste à des temps courts devant $t_{\mathrm{opt}}$. Le lecteur trouvera en fin d'Annexe B une extension de ces lois d'échelle au-delà du modèle à un mode, c'est-à-dire pour un rapport $\Gamma_{\mathrm{ex}} / \gamma_{f}$ quelconque, non infinitésimal; c'est elle qui a été retenue dans le résumé de l'article. Le lien entre $\mathcal{V}_{\text {opt }}$ et la coopérativité (107) est alors rompu.

Remerciements : Alice Sinatra remercie Franck Laloë pour d'utiles discussions. Tous les auteurs à l'exception d'Yvan Castin bénéficient d'un financement par le projet européen de recherche et d'innovation Horizon 2020 macQsimal numéro 820393. Matteo Fadel remercie le Fonds de recherche de l'Université de Bâle pour les jeunes chercheurs d'excellence.

\section{Annexe A. Traitement semi-classique et réduction à trois spins couplés}

Nous donnons ici les équations non linéaires qui décrivent la dynamique du système dans la théorie semi-classique, et nous les linéarisons pour de faibles fluctuations autour d'une solution stationnaire partiellement polarisée.

Équations semi-classiques non linéaires. En partant des considérations et des notations de la section 2, nous prenons la moyenne des équations du mouvement de Heisenberg dans l'état quantique du système et effectuons l'approximation de décorrélation (dite semi-classique en optique quantique) $\langle A B\rangle \simeq\langle A\rangle\langle B\rangle$ où $A$ et $B$ sont deux opérateurs, pour obtenir les équations d'évolution non linéaires suivantes sur les moyennes de $\vec{S}$ spin de Stokes du champ lumineux en cavité, $\vec{I}$ spin nucléaire collectif dans l'état fondamental, $\vec{J}$ et $\vec{K}$ spins collectifs associés aux multiplicités $F=3 / 2$ et $F=1 / 2$ dans l'état métastable, et $\overrightarrow{\vec{Q}}$ tenseur d'alignement collectif dans $F=3 / 2$, de composantes cartésiennes $Q_{\alpha \beta}$ :

$$
\begin{array}{rlrl}
\frac{\mathrm{d}\left\langle S_{x}\right\rangle}{\mathrm{d} t} & =-\frac{\kappa}{2}\left(\left\langle S_{x}\right\rangle-\frac{n_{\mathrm{ph}}}{2}\right)-\chi\left\langle K_{z}\right\rangle\left\langle S_{y}\right\rangle & \frac{\mathrm{d}\left\langle S_{y}\right\rangle}{\mathrm{d} t}=-\frac{\kappa}{2}\left\langle S_{y}\right\rangle+\chi\left\langle K_{z}\right\rangle\left\langle S_{x}\right\rangle & \frac{\mathrm{d}\left\langle S_{z}\right\rangle}{\mathrm{d} t}=-\frac{\kappa}{2}\left\langle S_{z}\right\rangle \\
\frac{\mathrm{d}\left\langle K_{x}\right\rangle}{\mathrm{d} t}=\left.\frac{\mathrm{d}\left\langle K_{x}\right\rangle}{\mathrm{d} t}\right|_{\mathrm{ECH}}-\chi\left\langle K_{y}\right\rangle\left\langle S_{z}\right\rangle & \frac{\mathrm{d}\left\langle K_{y}\right\rangle}{\mathrm{d} t}=\left.\frac{\mathrm{d}\left\langle K_{y}\right\rangle}{\mathrm{d} t}\right|_{\mathrm{ECH}}+\chi\left\langle K_{x}\right\rangle\left\langle S_{z}\right\rangle & \frac{\mathrm{d}\left\langle K_{z}\right\rangle}{\mathrm{d} t}=\left.\frac{\mathrm{d}\left\langle K_{z}\right\rangle}{\mathrm{d} t}\right|_{\mathrm{ECH}} \\
\frac{\mathrm{d}\langle\vec{J}\rangle}{\mathrm{d} t}=\left.\frac{\mathrm{d}\langle\vec{J}\rangle}{\mathrm{d} t}\right|_{\mathrm{ECH}} & \frac{\mathrm{d}\left\langle Q_{\alpha \beta}\right\rangle}{\mathrm{d} t}=\left.\frac{\mathrm{d}\left\langle Q_{\alpha \beta}\right\rangle}{\mathrm{d} t}\right|_{\mathrm{ECH}} \frac{\mathrm{d}\langle\vec{I}\rangle}{\mathrm{d} t}=\left.\frac{\mathrm{I}\rangle}{\mathrm{d} t}\right|_{\mathrm{ECH}}
\end{array}
$$


Les termes proportionnels au taux de perte $\kappa$ du miroir de sortie de la cavité font relaxer $\left\langle S_{x}\right\rangle$ vers sa valeur stationnaire $\left\langle S_{x}\right\rangle_{s}=n_{\mathrm{ph}} / 2$ forcée par le champ laser polarisé selon $O x$ injecté dans la cavité, et les moyennes transverses $\left\langle S_{y}\right\rangle$ et $\left\langle S_{z}\right\rangle$ vers zéro. Les termes proportionnels au couplage de Faraday $\chi$ entre le mode de cavité et le spin $\vec{K}$ dérivent de l'hamiltonien (2). La contribution des collisions d'échange de métastabilité (ECH) entre atomes fondamentaux et métastables se déduit directement de l'équation pilote sur l'opérateur densité à un atome des références [21, 22] par simple multiplication ou division par le nombre total de fondamentaux $N_{\text {cell }}$ ou métastables $n_{\text {cell }}$ dans la cellule : ${ }^{26}$

$$
\begin{aligned}
\left.\frac{\mathrm{d}\langle\vec{K}\rangle}{\mathrm{d} t}\right|_{\mathrm{ECH}} & =-\frac{7}{9 \tau}\langle\vec{K}\rangle+\frac{1}{9 \tau}\langle\vec{J}\rangle-\frac{1}{9 \tau} \frac{n_{\text {cell }}}{N_{\text {cell }}}\langle\vec{I}\rangle-\frac{4}{3 \tau} \frac{1}{N_{\text {cell }}}\langle\vec{Q}\rangle \cdot\langle\vec{I}\rangle \\
\left.\frac{\mathrm{d}\langle\vec{J}\rangle}{\mathrm{d} t}\right|_{\mathrm{ECH}} & =-\frac{4}{9 \tau}\langle\vec{J}\rangle+\frac{10}{9 \tau}\langle\vec{K}\rangle+\frac{10}{9 \tau} \frac{n_{\text {cell }}}{N_{\text {cell }}}\langle\vec{I}\rangle+\frac{4}{3 \tau} \frac{1}{N_{\text {cell }}}\langle\overrightarrow{\vec{Q}}\rangle \cdot\langle\vec{I}\rangle \\
\left.\frac{\mathrm{d}\left\langle Q_{\alpha \beta}\right\rangle}{\mathrm{d} t}\right|_{\mathrm{ECH}} & =-\frac{2}{3 \tau}\left\langle Q_{\alpha \beta}\right\rangle+\frac{1}{9 \tau} \frac{1}{N_{\text {cell }}}\left(3 \frac{\left\langle I_{\alpha}\right\rangle\left\langle\Sigma_{\beta}\right\rangle+\left\langle I_{\beta}\right\rangle\left\langle\Sigma_{\alpha}\right\rangle}{2}-\delta_{\alpha \beta}\langle\vec{I}\rangle \cdot\langle\vec{\Sigma}\rangle\right) \\
\left.\frac{\mathrm{d}\langle\vec{I}\rangle}{\mathrm{d} t}\right|_{\mathrm{ECH}} & =-\frac{1}{T}\langle\vec{I}\rangle+\frac{1}{3 T} \frac{N_{\text {cell }}}{n_{\text {cell }}}(\langle\vec{J}\rangle-\langle\vec{K}\rangle)
\end{aligned}
$$

où $\langle\vec{\Sigma}\rangle=\frac{2}{3}[\langle\vec{J}\rangle+2\langle\vec{K}\rangle]$ est la valeur moyenne du spin électronique dans l'état métastable. On se reportera aux équations (1.37b), (1.37a), (1.39) et (1.25) de la référence [22] (en tenant compte d'un écart d'un facteur 6 sur la définition du tenseur d'alignement), ou aux équations (VIII.30), (VIII.29), (VIII.32) et (VIII.15) de la référence [21] (en ajoutant un facteur de Kronecker $\delta_{\alpha \beta}$ omis dans (VIII.32)). Ici $1 / \tau$ et $1 / T$, les taux individuels des collisions d'échange de métastabilité subies par un atome dans l'état métastable et dans l'état fondamental, sont dans le rapport $T / \tau=N_{\text {cell }} / n_{\text {cell }}$ puisque, dans l'unité de temps, un nombre égal d'atomes fondamentaux et d'atomes métastables ont subi une collision d'échange [21, 22].

Solution stationnaire partiellement polarisée. Dans un état stationnaire polarisé de polarisation nucléaire $\eta \in[0,1]$,

$$
\left\langle I_{x}\right\rangle_{s}=\eta \frac{N_{\text {cell }}}{2} \quad ; \quad\left\langle I_{y}\right\rangle_{s}=\left\langle I_{z}\right\rangle_{s}=0 \quad ; \quad\left\langle S_{x}\right\rangle_{s}=\frac{n_{\mathrm{ph}}}{2} \quad ; \quad\left\langle S_{y}\right\rangle_{s}=\left\langle S_{z}\right\rangle_{s}=0
$$

l'invariance par rotation d'axe $O x$ contraint les spins moyens dans l'état métastable à être alignés selon $O x$, et le tenseur d'alignement moyen à être diagonal dans la base cartésienne, avec des valeurs propres égales selon les directions $O y$ et $O z$. Le système (A.1)-(A.3) admet ainsi une solution stationnaire avec comme seules moyennes non nulles dans l'état métastable :

$$
\left\langle K_{x}\right\rangle_{s}=\frac{\eta}{2} \frac{1-\eta^{2}}{3+\eta^{2}} n_{\text {cell }} ;\left\langle J_{x}\right\rangle_{s}=\eta \frac{5+\eta^{2}}{3+\eta^{2}} n_{\text {cell }} ;\left\langle\Sigma_{x}\right\rangle_{s}=\frac{4 \eta}{3+\eta^{2}} n_{\text {cell }} ;\left\langle Q_{y y}\right\rangle_{s}=\left\langle Q_{z z}\right\rangle_{s}=-\frac{1}{2}\left\langle Q_{x x}\right\rangle_{s}=-\frac{\eta}{12}\left\langle\Sigma_{x}\right\rangle_{s}
$$

Équations semi-classiques linéarisées. On linéarise maintenant les équations (A.1)-(A.3) en les fluctuations classiques autour de la solution stationnaire (A.8)-(A.9) en effectuant la substitution $\langle A\rangle \rightarrow\langle A\rangle_{s}+\delta A$ et en traitant $\delta A$ au premier ordre. En nous limitant au sous-espace des fluctuations transverses, c'est-à-dire aux directions $\alpha=y, z$

\footnotetext{
26. Les moyennes collectives sont en effet reliées comme suit aux moyennes à un atome \langle\rangle$_{\text {at }}:\langle\vec{I}\rangle=N_{\text {cell }}\langle\vec{I}\rangle_{\text {at }},\langle\vec{J}\rangle=n_{\text {cell }}\langle\vec{J}\rangle_{\text {at }},\langle\vec{K}\rangle=n_{\text {cell }}\langle\vec{K}\rangle_{\text {at }}$, $\langle\vec{Q}\rangle=n_{\text {cell }}\langle\vec{Q}\rangle_{\text {at }},\langle\vec{\Sigma}\rangle=n_{\text {cell }}\langle\vec{\Sigma}\rangle_{\text {at }}$.
} 
orthogonales aux spins moyens, nous obtenons un système fermé :

$$
\begin{aligned}
\frac{\mathrm{d}}{\mathrm{d} t} \delta S_{\alpha} & =-\frac{\kappa}{2} \delta S_{\alpha}+\chi \delta_{\alpha y}\left\langle S_{x}\right\rangle_{s} \delta K_{z} \\
\frac{\mathrm{d}}{\mathrm{d} t} \delta K_{\alpha} & =-\frac{7}{9 \tau} \delta K_{\alpha}+\frac{1}{9 \tau} \delta J_{\alpha}-\frac{2 \eta}{3 \tau} \delta Q_{\alpha x}-\frac{1}{9 T}\left(1+\frac{12}{n_{\text {cell }}}\left\langle Q_{\alpha \alpha}\right\rangle_{s}\right) \delta I_{\alpha}+\chi \delta_{\alpha y}\left\langle K_{x}\right\rangle_{s} \delta S_{z} \\
\frac{\mathrm{d}}{\mathrm{d} t} \delta J_{\alpha} & =-\frac{4}{9 \tau} \delta J_{\alpha}+\frac{10}{9 \tau} \delta K_{\alpha}+\frac{2 \eta}{3 \tau} \delta Q_{\alpha x}+\frac{10}{9 T}\left(1+\frac{6}{5 n_{\text {cell }}}\left\langle Q_{\alpha \alpha}\right\rangle\right) \delta I_{\alpha} \\
\frac{\mathrm{d}}{\mathrm{d} t} \delta Q_{\alpha x} & =-\frac{2}{3 \tau} \delta Q_{\alpha x}+\frac{\eta}{12 \tau} \delta \Sigma_{\alpha}+\frac{1}{6 T n_{\text {cell }}}\left\langle\Sigma_{x}\right\rangle_{s} \delta I_{\alpha} \\
\frac{\mathrm{d}}{\mathrm{d} t} \delta I_{\alpha} & =-\frac{1}{T} \delta I_{\alpha}+\frac{1}{3 \tau}\left(\delta J_{\alpha}-\delta K_{\alpha}\right)
\end{aligned}
$$

Réduction à trois spins collectifs couplés. En posant $\frac{\mathrm{d}}{\mathrm{d} t} \delta J_{\alpha}=0$ dans l'équation (A.12) et $\frac{\mathrm{d}}{\mathrm{d} t} \delta Q_{\alpha x}=0$ dans l'équation (A.13), nous éliminons adiabatiquement les fluctuations du spin collectif $\vec{J}$ et du tenseur d'alignement collectif dont les évolutions sont régies par l'échange de métastabilité uniquement :

$$
\delta J_{\alpha}^{\text {adiab }}=2 \frac{10+\eta^{2}}{8-\eta^{2}} \delta K_{\alpha}+\frac{12 \tau}{T} \frac{5+2 \eta^{2}}{\left(3+\eta^{2}\right)\left(8-\eta^{2}\right)} \delta I_{\alpha} \quad ; \quad \delta Q_{\alpha x}^{\text {adiab }}=\frac{3 \eta}{8-\eta^{2}} \delta K_{\alpha}+\frac{\tau}{T} \frac{\eta\left(13+\eta^{2}\right)}{\left(3+\eta^{2}\right)\left(8-\eta^{2}\right)} \delta I_{\alpha}
$$

Le report des expressions adiabatiques (A.15) dans les équations (A.11) et (A.14) sur $\delta K_{\alpha}$ et $\delta I_{\alpha}$ conduit dans le corps de l'article au système réduit (5)-(7) couplant les fluctuations des trois spins (3), où $\gamma_{f}$ et $\gamma_{m}$, les taux effectifs d'échange de métastabilité entre le spin nucléaire et le spin $F=1 / 2$ du métastable, sont donnés par l'équation (8).

\section{Annexe B. Solution du modèle à trois modes avec décohérence pour la détection homodyne}

Nous donnons ici la solution analytique du modèle à trois modes en présence de décohérence, voir l'équation pilote (97), pour une évolution du système conditionnée à une mesure homodyne en continu de la composante polarisée selon $O y$ du champ sortant de la cavité. La valeur des coefficients $\gamma_{\alpha}, \gamma_{\beta}, \Omega_{\alpha}$ et $\Omega_{\beta}$, ainsi que des opérateurs d'annihilation $\alpha$ et $\beta$, se déduit d'une diagonalisation de la matrice des taux (96). Les taux $\gamma_{\alpha}$ et $\gamma_{\beta}$ en sont les valeurs propres rangées par ordre croissant :

$$
\gamma_{\alpha, \beta}=\gamma_{m}+\gamma_{f}+\gamma_{0} \mp\left[\left(\gamma_{m}+\gamma_{f}+\gamma_{0}\right)^{2}-4 \gamma_{f} \gamma_{0}\right]^{1 / 2}
$$

En termes des pulsations de Faraday $\Omega_{\alpha}$ et $\Omega_{\beta}$, les vecteurs propres normalisés correspondants s'écrivent $\left(\Omega_{\beta} / \Omega, \Omega_{\alpha} / \Omega\right)$ et $\left(-\Omega_{\alpha} / \Omega, \Omega_{\beta} / \Omega\right)$, si bien que $\alpha=\left(\Omega_{\beta} a+\Omega_{\alpha} b\right) / \Omega$ et $\beta=\left(\Omega_{\beta} b-\Omega_{\alpha} a\right) / \Omega$ avec

$$
\Omega_{\alpha}=\frac{\Omega\left(\gamma_{f}-\gamma_{\alpha} / 2\right)}{\left[\gamma_{m} \gamma_{f}+\left(\gamma_{f}-\gamma_{\alpha} / 2\right)^{2}\right]^{1 / 2}} \quad ; \quad \Omega_{\beta}=\frac{\Omega \sqrt{\gamma_{m} \gamma_{f}}}{\left[\gamma_{m} \gamma_{f}+\left(\gamma_{f}-\gamma_{\alpha} / 2\right)^{2}\right]^{1 / 2}}
$$

dans un choix de signe assurant que $\alpha \rightarrow a$ et $\beta \rightarrow b$ lorsque $\gamma_{f} \rightarrow 0$ et reproduisant (26) lorsque $\gamma_{0} \rightarrow 0$. Puisque l'opérateur de saut $C_{\alpha} \propto \alpha$ décrit des processus non mesurés, nous pouvons, comme nous l'avons fait pour $C_{\beta}$, le prendre de la forme $\sqrt{\gamma_{\alpha}} \mathrm{i} \alpha$ et réutiliser l'ansatz gaussien réel (73) afin de résoudre l'équation stochastique (55) sur le vecteur d'état. Dans l'équation d'évolution de la matrice $\underline{u}$ apparaissant dans l'ansatz, les indices $\alpha$ et $\beta$ jouent désormais des rôles symétriques et l'on obtient

$$
\begin{array}{lll}
\mathrm{d} u_{\alpha \alpha}=-\Omega_{\alpha} \mathrm{d} t u_{\alpha c}+\gamma_{\alpha} \mathrm{d} t\left(1-u_{\alpha \alpha}\right) & \mathrm{d} u_{\alpha \beta}=-\frac{\mathrm{d} t}{2}\left[\left(\gamma_{\alpha}+\gamma_{\beta}\right) u_{\alpha \beta}+\Omega_{\beta} u_{\alpha c}+\Omega_{\alpha} u_{\beta c}\right] & \mathrm{d} u_{\alpha c}=-\frac{\mathrm{d} t}{2}\left[\left(\gamma_{\alpha}+\kappa\right) u_{\alpha c}+\Omega_{\alpha} u_{c c}\right] \\
\mathrm{d} u_{\beta \beta}=-\Omega_{\beta} \mathrm{d} t u_{\beta c}+\gamma_{\beta} \mathrm{d} t\left(1-u_{\beta \beta}\right) & \mathrm{d} u_{\beta c}=-\frac{\mathrm{d} t}{2}\left[\left(\gamma_{\beta}+\kappa\right) u_{\beta c}+\Omega_{\beta} u_{c c}\right] & \mathrm{d} u_{c c}=\kappa \mathrm{d} t\left(1-u_{c c}\right)
\end{array}
$$


dont la solution pour la condition initiale $\underline{\underline{u}}(0)=$ Id s'écrit

$$
\begin{aligned}
u_{\alpha \alpha}(t)= & 1+\frac{\Omega_{\alpha}^{2}}{\gamma_{\alpha}\left(\kappa+\gamma_{\alpha}\right)}\left(1-\mathrm{e}^{-\gamma_{\alpha} t}\right)-\frac{2 \Omega_{\alpha}^{2}}{\kappa^{2}-\gamma_{\alpha}^{2}}\left(\mathrm{e}^{-\gamma_{\alpha} t}-\mathrm{e}^{-\left(\kappa+\gamma_{\alpha}\right) t / 2}\right) \\
u_{\alpha \beta}(t)= & \frac{\Omega_{\alpha} \Omega_{\beta}}{\gamma_{\alpha}+\gamma_{\beta}}\left(\frac{1}{\kappa+\gamma_{\alpha}}+\frac{1}{\kappa+\gamma_{\beta}}\right)\left(1-\mathrm{e}^{-\left(\gamma_{\alpha}+\gamma_{\beta}\right) t / 2}\right)+\frac{\Omega_{\alpha} \Omega_{\beta}}{\left(\kappa-\gamma_{\beta}\right)\left(\kappa+\gamma_{\alpha}\right)}\left(\mathrm{e}^{-\left(\kappa+\gamma_{\alpha}\right) t / 2}-\mathrm{e}^{-\left(\gamma_{\alpha}+\gamma_{\beta}\right) t / 2}\right) \\
& +\frac{\Omega_{\alpha} \Omega_{\beta}}{\left(\kappa-\gamma_{\alpha}\right)\left(\kappa+\gamma_{\beta}\right)}\left(\mathrm{e}^{-\left(\kappa+\gamma_{\beta}\right) t / 2}-\mathrm{e}^{-\left(\gamma_{\alpha}+\gamma_{\beta}\right) t / 2}\right) \\
u_{\alpha c}(t)= & -\frac{\Omega_{\alpha}}{\kappa+\gamma_{\alpha}}\left(1-\mathrm{e}^{-\left(\kappa+\gamma_{\alpha}\right) t / 2}\right) \\
u_{\beta \beta}(t)= & 1+\frac{\Omega_{\beta}^{2}}{\gamma_{\beta}\left(\kappa+\gamma_{\beta}\right)}\left(1-\mathrm{e}^{-\gamma_{\beta} t}\right)-\frac{2 \Omega_{\beta}^{2}}{\kappa^{2}-\gamma_{\beta}^{2}}\left(\mathrm{e}^{-\gamma_{\beta} t}-\mathrm{e}^{-\left(\kappa+\gamma_{\beta}\right) t / 2}\right) \\
u_{\beta c}(t)= & -\frac{\Omega_{\beta}}{\kappa+\gamma_{\beta}}\left(1-\mathrm{e}^{-\left(\kappa+\gamma_{\beta}\right) t / 2}\right) \\
u_{c c}(t)= & 1
\end{aligned}
$$

Le vecteur des coordonnées moyennes $\overline{\mathbf{q}}$ apparaissant dans l'ansatz (73) obéit à l'équation stochastique

$$
\mathrm{d} \overline{\mathbf{q}}=\frac{1}{2}\left(\begin{array}{ccc}
-\gamma_{\alpha} & 0 & 0 \\
0 & -\gamma_{\beta} & 0 \\
\Omega_{\alpha} & \Omega_{\beta} & -\kappa
\end{array}\right) \mathrm{d} t \overline{\mathbf{q}}+\frac{1}{2}[\mathrm{Id}-\underline{\underline{c}}(t)]\left(\begin{array}{c}
\gamma_{\alpha}^{1 / 2} \mathrm{~d} \zeta_{\alpha}(t) \\
\gamma_{\beta}^{1 / 2} \mathrm{~d} \zeta_{\beta}(t) \\
-\kappa^{1 / 2} \mathrm{~d} \zeta_{c}(t)
\end{array}\right)
$$

La moyenne non conditionnelle $\left\langle P_{a}^{2}\right\rangle$ valant toujours $1 / 4$, la moyenne et la variance de $P_{a}$ conditionnées au signal homodyne intégré restent données par les équations (85) et (86), en généralisant les expressions (92) des variances et covariance des variables aléatoires $\bar{P}_{a}(t)$ et $\sigma(t)$ au cas de trois processus stochastiques indépendants d $\zeta_{\alpha}\left(t^{\prime}\right)$, d $\zeta_{\beta}\left(t^{\prime}\right)$ et $\mathrm{d} \zeta_{c}\left(t^{\prime}\right)$ comme suit :

$$
\begin{aligned}
\left\langle\sigma(t) \bar{P}_{a}(t)\right\rangle_{\text {stoch }} & =\int_{0}^{t} \mathrm{~d} t^{\prime} \sum_{v \in\{\alpha, \beta, c\}} p_{v}\left(t, t^{\prime}\right) \sigma_{v}\left(t, t^{\prime}\right) ;\left\langle\sigma^{2}(t)\right\rangle_{\text {stoch }}=\int_{0}^{t} \mathrm{~d} t^{\prime} \sum_{v \in\{\alpha, \beta, c\}} \sigma_{v}^{2}\left(t, t^{\prime}\right) ; \\
\left\langle\bar{P}_{a}^{2}(t)\right\rangle_{\text {stoch }} & =\int_{0}^{t} \mathrm{~d} t^{\prime} \sum_{v \in\{\alpha, \beta, c\}} p_{v}^{2}\left(t, t^{\prime}\right)
\end{aligned}
$$

avec les expressions compactes des amplitudes correspondantes

$$
\begin{aligned}
& p_{v}\left(t, t^{\prime}\right)=(-1)^{\delta_{v c}} \frac{\sqrt{\gamma_{v}}}{2 \Omega}\left\{\Omega_{\beta} \mathrm{e}^{-\gamma_{\alpha}\left(t-t^{\prime}\right) / 2}\left[\delta_{\alpha v}-c_{\alpha v}\left(t^{\prime}\right)\right]-\Omega_{\alpha} \mathrm{e}^{-\gamma_{\beta}\left(t-t^{\prime}\right) / 2}\left[\delta_{\beta v}-c_{\beta v}\left(t^{\prime}\right)\right]\right\} \\
& \sigma_{v}\left(t, t^{\prime}\right)=\frac{\delta_{v c}}{2 t}+(-1)^{\delta_{v c}} \frac{\sqrt{\kappa \gamma_{\nu}}}{2 t}\left\{\left[\delta_{c v}-c_{c v}\left(t^{\prime}\right)\right] f_{\kappa}\left(t-t^{\prime}\right)+\sum_{\mu \in\{\alpha, \beta\}} \frac{\Omega_{\mu}}{\kappa-\gamma_{\mu}}\left[\delta_{\mu v}-c_{\mu v}\left(t^{\prime}\right)\right]\left[f_{\gamma_{\mu}}\left(t-t^{\prime}\right)-f_{\kappa}\left(t-t^{\prime}\right)\right]\right\}
\end{aligned}
$$

L'indice $v$ court sur les trois valeurs $\alpha, \beta, c$ et l'on a posé $\gamma_{c}=\kappa$. La fonction $\delta$ est celle de Kronecker, et la fonction $f_{\lambda}$ est la même que dans les équations (88)-(91).

La solution générale que nous venons d'exposer comporte les cinq taux $\gamma_{\alpha}, \Gamma_{\mathrm{ex}}=\Omega_{\alpha}^{2} / \kappa, \gamma_{f}$ d'une part, $\gamma_{\beta}, \kappa \mathrm{d}$ 'autre part. Le régime pertinent expérimentalement est celui où les deux derniers sont «infiniment » plus grands que les trois premiers et ne contribuent qu'au travers de régimes transitoires inobservables. Mathématiquement, on accède à cette limite en faisant tendre $\gamma_{f}$ vers zéro à $\kappa, \gamma_{m}, \gamma_{0}$ et $\Omega$ fixés et à $\tau=\Gamma_{\text {ex }} t>0$ fixé. Alors les trois premiers taux tendent conjointement vers zéro, c'est-à-dire dans des rapports de limite finie et non nulle $\Gamma_{\mathrm{ex}} / \gamma_{f} \rightarrow \Omega^{2} \gamma_{m} /\left[\kappa\left(\gamma_{0}+\gamma_{m}\right)^{2}\right]$ et $\gamma_{\alpha} / \gamma_{f} \rightarrow 2 \gamma_{0} /\left(\gamma_{0}+\gamma_{m}\right)$, le taux $\gamma_{\beta}$ se réduit à $\gamma \equiv 2\left(\gamma_{0}+\gamma_{m}\right)$ et le couplage de Faraday $\Omega_{\beta}$ à $\Omega$. Tous les transitoires exponentiels disparaissent dans les éléments de matrice (B.4)-(B.8) de $\underline{u}$ sauf ceux relaxant au taux $\gamma_{\alpha}$. Les amplitudes 
(B.12) et (B.13) sur les processus stochastiques se réduisent à

$$
\begin{aligned}
\frac{p_{\alpha}\left(t, t^{\prime}\right)}{\sqrt{\Gamma_{\mathrm{ex}}}}=\frac{u\left(\tau^{\prime}\right)-1}{2 u\left(\tau^{\prime}\right)} \sqrt{\epsilon} \mathrm{e}^{-\epsilon\left(\tau-\tau^{\prime}\right) / 2} & \frac{\sigma_{\alpha}\left(t, t^{\prime}\right)}{\Gamma_{\mathrm{ex}}}=\frac{u\left(\tau^{\prime}\right)-1}{\tau u\left(\tau^{\prime}\right)} \sqrt{\epsilon} \frac{1-\mathrm{e}^{-\epsilon\left(\tau-\tau^{\prime}\right) / 2}}{\epsilon} \\
\frac{p_{\beta}\left(t, t^{\prime}\right)}{\sqrt{\Gamma_{\mathrm{ex}}}}=\frac{\sqrt{\rho}}{(1+\rho) u\left(\tau^{\prime}\right)} \mathrm{e}^{-\epsilon\left(\tau-\tau^{\prime}\right) / 2} & \frac{\sigma_{\beta}\left(t, t^{\prime}\right)}{\Gamma_{\mathrm{ex}}}=\frac{\sqrt{\rho}}{(1+\rho) \tau}\left[\frac{2}{u\left(\tau^{\prime}\right)} \frac{1-\mathrm{e}^{-\epsilon\left(\tau-\tau^{\prime}\right) / 2}}{\epsilon}+\rho+\frac{\gamma}{\kappa}(\rho-1)\right] \\
\frac{p_{c}\left(t, t^{\prime}\right)}{\sqrt{\Gamma_{\mathrm{ex}}}}=\frac{(1-\rho)}{2(1+\rho) u\left(\tau^{\prime}\right)} \mathrm{e}^{-\epsilon\left(\tau-\tau^{\prime}\right) / 2} & \frac{\sigma_{c}\left(t, t^{\prime}\right)}{\Gamma_{\mathrm{ex}}}=\frac{1}{\tau}\left[\frac{1}{2}+\frac{1-\rho}{1+\rho} \frac{1}{u\left(\tau^{\prime}\right)} \frac{1-\mathrm{e}^{-\epsilon\left(\tau-\tau^{\prime}\right) / 2}}{\epsilon}+\frac{\rho}{1+\rho}\left(1+\frac{2 \gamma}{\kappa}\right)\right]
\end{aligned}
$$

où $\epsilon=\gamma_{\alpha} / \Gamma_{\mathrm{ex}}$ comme dans la section 4.2.4, la fonction $u(\tau)$ est donnée par l'équation (100) et la notation $\rho=$ $\Omega^{2} \kappa /\left[\gamma(\kappa+\gamma)^{2}\right]$ généralise celle de la note 22. Les relations (85) et (86) restent valables, avec les nouvelles expressions des variance et covariance

$$
\frac{\left\langle\sigma^{2}\right\rangle_{\mathrm{stoch}}}{\Gamma_{\mathrm{ex}}}=\frac{\epsilon \tau-2\left(1-\mathrm{e}^{-\epsilon \tau / 2}\right)}{\epsilon^{2} \tau^{2}}+\frac{\Gamma_{\mathrm{ex}}}{4 \tau \Gamma_{\mathrm{sq}}^{\mathrm{gen}}} \quad \text { et } \quad \frac{\left\langle\sigma \bar{P}_{a}\right\rangle_{\mathrm{stoch}}}{\sqrt{\Gamma_{\mathrm{ex}}}}=\frac{1-\mathrm{e}^{-\epsilon \tau / 2}}{2 \epsilon \tau}
$$

et du taux de compression généralisé

$$
\Gamma_{\mathrm{sq}}^{\mathrm{gen}}=\left[\frac{1}{\Gamma_{\mathrm{ex}}}+\frac{2\left(\gamma_{0}+\gamma_{m}\right)}{\gamma_{f} \gamma_{m}}\right]^{-1}
$$

qui reproduisent les variance et covariance (104) et (105) du modèle à un mode avec décohérence lorsque $\Gamma_{\mathrm{ex}} / \gamma_{f} \rightarrow 0$ et le taux de compression de spin (95) du modèle à trois modes sans décohérence lorsque $\gamma_{0} \rightarrow 0$. Les nouveaux résultats peuvent être simplifiés dans la limite utile de faible décohérence ramenée $\gamma_{\alpha} / \Gamma_{\mathrm{ex}} \rightarrow 0$ par un développement à l'ordre un en $\epsilon$, ce qui permet de généraliser comme suit les résultats (106) sur la moyenne et la variance conditionnelles à une valeur de $\Gamma_{\mathrm{ex}} / \gamma_{f}$ non infinitésimale :

$$
\begin{aligned}
& m(t)=\frac{\Gamma_{\mathrm{sq}}^{\mathrm{gen}} t}{1+\Gamma_{\mathrm{sq}}^{\mathrm{gen}} t}-\frac{\gamma_{\alpha}}{\Gamma_{\mathrm{sq}}^{\mathrm{gen}}} \frac{\left(3+\Gamma_{\mathrm{sq}}^{\mathrm{gen}} t\right)\left(\Gamma_{\mathrm{sq}}^{\mathrm{gen}} t\right)^{2}}{12\left(1+\Gamma_{\mathrm{sq}}^{\mathrm{gen}} t\right)^{2}}+O\left[\left(\gamma_{\alpha} t\right)^{2}\right] \\
& \mathcal{V}(t)=\frac{1}{4\left(1+\Gamma_{\mathrm{sq}}^{\mathrm{gen}} t\right)}+\frac{\gamma_{\alpha}}{\Gamma_{\mathrm{sq}}^{\mathrm{gen}}} \frac{\left(\Gamma_{\mathrm{sq}}^{\mathrm{gen}} t+3 / 2\right)\left(\Gamma_{\mathrm{sq}}^{\mathrm{gen}} t\right)^{2}}{12\left(1+\Gamma_{\mathrm{sq}}^{\mathrm{gen}} t\right)^{2}}+O\left[\left(\gamma_{\alpha} t\right)^{2}\right]
\end{aligned}
$$

Cette généralisation revient simplement à remplacer $\tau$ par $\Gamma_{\mathrm{sq}}^{\mathrm{gen}} t$ et $\epsilon$ par $\gamma_{\alpha} / \Gamma_{\mathrm{sq}}^{\mathrm{gen}}$ dans les seconds membres de (106). ${ }^{27}$ La compression optimale sur $P_{a}$ est alors obtenue au bout d'un temps $t_{\mathrm{opt}} \sim\left(3 / \Gamma_{\mathrm{sq}}^{\mathrm{gen}} \gamma_{\alpha}\right)^{1 / 2}$ et correspond à une variance conditionnelle $\operatorname{Var}_{\sigma=\mathcal{S}}^{\mathrm{opt}}\left(P_{a}\right) \sim\left(\gamma_{\alpha} / 12 \Gamma_{\mathrm{sq}}^{\mathrm{gen}}\right)^{1 / 2}$; le gain métrologique optimal s'en déduit par l'équation (13).

\section{Références}

[1] J. MacFall, H. Charles, R. Black, H. Middleton, J. Swartz, B. Saam, B. Driehuys, C. Erickson, W. Happer, G. Cates, G. Johnson, C. Ravin, «Human lung air spaces: potential for MR imaging with hyperpolarized He-3», Radiology 200 (1996), p. 553.

[2] C. Gemmel, W. Heil, S. Karpuk, K. Lenz, C. Ludwig, Y. Sobolev, K. Tullney, M. Burghoff, W. Kilian, S. Knappe-Grüneberg, W. Müller, A. Schnabel, F. Seifert, L. Trahms, S. Baeßler, «Ultra-sensitive magnetometry based on free precession of nuclear spins », Eur. Phys. J. D 57 (2010), p. 303.

[3] T. R. Gentile, P. J. Nacher, B. Saam, T. G. Walker, « Optically polarized ${ }^{3} \mathrm{He}$ », Rev. Mod. Phys. 89 (2017), 045004.

[4] L. Pezzè, A. Smerzi, M. K. Oberthaler, R. Schmied, P. Treutlein, «Quantum metrology with nonclassical states of atomic ensembles », Rev. Mod. Phys. 90 (2018), 035005.

[5] A. Dantan, G. Reinaudi, A. Sinatra, F. Laloë, E. Giacobino, M. Pinard, «Long-Lived Quantum Memory with Nuclear Atomic Spins », Phys. Rev. Lett. 95 (2005), 123002.

[6] G. Reinaudi, A. Sinatra, A. Dantan, M. Pinard, « Squeezing and entangling nuclear spins in helium 3 », Journal of Modern Optics 54 (2007), p. 675 .

27. Elle vaut en fait à tous les ordres en $\epsilon$ puisque le remplacement proposé ne change pas $\epsilon \tau$ (toujours égal à $\gamma_{\alpha} t$ ) et fait passer des équations (104) et (105) à l'équation (B.17). 
[7] G. Vasilakis, H. Shen, K. Jensen, M. Balabas, D. Salart, B. Chen, E. Polzik, « Generation of a squeezed state of an oscillator by stroboscopic back-action-evading measurement », Nature Phys. 11 (2015), p. 389.

[8] O. Hosten, N. J. Engelsen, R. Krishnakumar, M. A. Kasevich, « Measurement noise 100 times lower than the quantum-projection limit using entangled atoms », Nature $\mathbf{5 2 9}$ (2016), p. 505.

[9] Han Bao, Junlei Duan, Shenchao Jin, Xingda Lu, Pengxiong Li, Weizhi Qu, Mingfeng Wang, I. Novikova, E.E. Mikhailov, Kai-Feng Zhao, K. Mølmer, Heng Shen, Yanhong Xiao, «Spin squeezing of $10^{11}$ atoms by prediction and retrodiction measurements », Nature $\mathbf{5 8 1}$ (2020), p. 159.

[10] M.-Z. Huang, J. A. de la Paz, T. Mazzoni, K. Ott, A. Sinatra, C. L. G. Alzar, J. Reichel, « Self-amplifying spin measurement in a long-lived spin-squeezed state », prépublication, arXiv:2007.01964 (2020).

[11] D.J. Wineland, J.J. Bollinger, W.M. Itano, D.J. Heinzen, Squeezed atomic states and projection noise in spectroscopy, Phys. Rev. A 50 (1994), p. 67.

[12] M. Kitagawa, M. Ueda, Squeezed spin states, Phys. Rev. A 47 (1993), p. 5138.

[13] G. Santarelli, Ph. Laurent, P. Lemonde, A. Clairon, A.G. Mann, S. Chang, A.N. Luiten, C. Salomon, Quantum Projection Noise in an Atomic Fountain: A High Stability Cesium Frequency Standard, Phys. Rev. Lett. 82 (1999), p.4619.

[14] A. Serafin, M. Fadel, P. Treutlein, A. Sinatra, « Nuclear spin squeezing in Helium-3 by continuous quantum non-demolition measurement », prépublication, hal-03058456 (2020).

[15] O. Katz, R. Shaham, E. S. Polzik, O. Firstenberg, «Long-Lived Entanglement Generation of Nuclear Spins Using Coherent Light », Phys. Rev. Lett. 124 (2020), 043602

[16] O. Katz, R. Shaham, O. Firstenberg, « Quantum interface for noble-gas spins », prépublication, arXiv:1905.12532 (2019).

[17] J.M. Geremia, J.K. Stockton, A.C. Doherty, H. Mabuchi, «Quantum Kalman Filtering and the Heisenberg Limit in Atomic Magnetometry », Phys. Rev. Lett. 91 (2003), 250801.

[18] K. Mølmer, L.B. Madsen, «Estimation of a classical parameter with Gaussian probes: Magnetometry with collective atomic spins », Phys. Rev. A 70 (2004), 052102

[19] R. Jiménez-Martínez, J. Kolodynski, C. Troullinou, V.G. Lucivero, Jia Kong, M.W. Mitchell, « Signal Tracking Beyond the Time Resolution of an Atomic Sensor by Kalman Filtering », Phys. Rev. Lett. 120 (2018), 040503.

[20] Cheng Zhang, K. Mølmer, «Estimating a fluctuating magnetic field with a continuously monitored atomic ensemble», Phys. Rev. A 102 (2020), 063716

[21] J. Dupont-Roc, «Étude de quelques effets liés au pompage optique en champ faible », Thèse, Université Paris VI, 1972.

[22] J. Dupont-Roc, M. Leduc, F. Laloë, «Contribution à l'étude du pompage optique par échange de métastabilité dans 3He. - Première Partie », Journal de Physique 34 (1973), p. 961.

[23] J. Cviklinski, A. Dantan, J. Ortalo, M. Pinard, «Conditional squeezing of an atomic alignment », Phys. Rev. A 76 (2007), 033830

[24] A. Kuzmich, L. Mandel, J. Janis, Y. E. Young, R. Ejnisman, N. P. Bigelow, «Quantum nondemolition measurements of collective atomic spin », Phys. Rev. A 60 (1999), p. 2346.

[25] F. Reiter, A.S. Sørensen, «Effective operator formalism for open quantum systems », Phys. Rev. A 85 (2012), 032111.

[26] Y. Castin, K. Mølmer, «Monte Carlo Wave-Function Analysis of 3D Optical Molasses », Phys. Rev. Lett. 74 (1995), p. 3772.

[27] K. Mølmer, Y. Castin, J. Dalibard, « Monte Carlo wave-function method in quantum optics », J. Opt. Soc. Am. B 10 (1993), p. 524.

[28] Y. Castin, J. Dalibard, K. Mølmer, «A Wave Function approach to dissipative processes », AIP Conference Proceedings, Thirteenth International Conference on Atomic Physics (édité par H. Walther, T.W. Hänsch, B. Neizert), 275 (1992).

[29] H. M. Wiseman, G. J. Milburn, «Quantum theory of field-quadrature measurements », Phys. Rev. A 47 (1993), p. 642.

[30] Yun Li, Y. Castin, A. Sinatra, «Optimum Spin Squeezing in Bose-Einstein Condensates with Particle Losses », Phys. Rev. Lett. 100 (2008), 210401.

[31] S. Zippilli, D. Vitali, P. Tombesi, J.-M. Raimond, « Scheme for decoherence control in microwave cavities », Phys. Rev. A 67 (2003), 052101.

[32] L. K. Thomsen, S. Mancini, H. M. Wiseman, «Continuous quantum nondemolition feedback and unconditional atomic spin squeezing », J. Phys. B 35 (2002), p. 4937.

[33] L.B. Madsen, K. Mølmer, «Spin squeezing and precision probing with light and samples of atoms in the Gaussian description », Phys. Rev. A 70 (2004), 052324.

[34] G. Lindblad, « On the Generators of Quantum dynamical semigroups », Commun. Math. Phys. 48 (1976), p. 119.

[35] N. Gisin, «Stochastic quantum dynamics and relativity », Helv. Phys. Acta 62 (1989), p. 363.

[36] N. Gisin, I. Percival, « The quantum state diffusion model applied to open systems », J. Phys. A 25 (1992), p. 5677.

[37] N. Gisin, «Quantum Measurements and Stochastic Processes », Phys. Rev. Lett. 52 (1984), p. 1657.

[38] J.K. Stockton, J.M. Geremia, A.C. Doherty, H. Mabuchi, « Robust quantum parameter estimation: Coherent magnetometry with feedback », Phys. Rev. A 69 (2004), 032109.

[39] H. Tanji-Suzuki, I.D. Leroux, M.H. Schleier-Smith, M. Cetina, A.T. Grier, J. Simon, V. Vuletic, « Interaction between Atomic Ensembles and Optical Resonators: Classical Description», Adv. At. Mol. Opt. Phys. 60 (2011), p. 201. 